\title{
Heat Transfer to a Moving Wire Immersed in a Gas Fluidized Bed Furnace
}

by

\author{
Antonio Tannas \\ Bachelor of Aerospace Engineering \\ Ryerson University, 2007
}

\begin{abstract}
A thesis presented to Ryerson University in partial fulfillment of the requirements

for the degree of

Master of Applied Science in the Program of Mechanical and Industrial Engineering
\end{abstract}

Toronto, Ontario, Canada, 2015

(C)Antonio Tannas 2015 


\section{Author's Declaration}

I hereby declare that I am the sole author of this thesis. This is a true copy of the thesis, including any required final revisions, as accepted by my examiners.

I authorize Ryerson University to lend this thesis to other institutions or individuals for the purpose of scholarly research.

I further authorize Ryerson University to reproduce this thesis by photocopying or by other means, in total or in part, at the request of other institutions or individuals for the purpose of scholarly research.

I understand that my thesis may be made electronically available to the public. 


\begin{abstract}
Heat Transfer to a Moving Wire Immersed in a Gas Fluidized Bed Furnace

Antonio Tannas, Master of Applied Science, Mechanical and Industrial Engineering, 2015

Ryerson University

In order to replace hazardous molten lead baths in the heat treatment of carbon steel wire with environmentally friendly fluidized bed furnaces a better understanding is needed of their heat transfer rates. There has been considerable work done in examining heat transfer rates to large cylinders immersed in fluidized beds, and some on wire sized ones as well, but all previous studies have been conducted on static cylinders. In order to gain a deeper understanding of heat transfer rates to a moving wire immersed in a fluidized bed furnace an apparatus has been constructed to move a wire through a fluidized bed. The heat transfer rates were calculated using the difference in inlet and outlet temperatures, wire speed and the bed temperature. As predicted, correlations for static wire were found to under-predict heat transfer rates at higher wire speeds, so a new correlation was developed by modifying an existing one.
\end{abstract}




\section{Acknowledgements}

I would like to thank my supervisors Dr. Jacob Friedman and Dr. David Naylor for guiding me and supporting me throughout this endeavour. When I first entered graduate school, I was filled with self-doubt and constantly questioned my abilities. They helped me find a confidence in my skills I had never had before, and helped foster a love of scientific research that has forever changed my outlook on life. They encouraged me to think on my own and to really take charge of my project, and never made me feel stupid about my mistakes. I now feel ready to take on any challenge, and am forever grateful to them for their guidance.

I'd also like to thank Joseph Amankrah for working diligently to bring my vision to life, and making me a better Engineer by teaching me many things about machining. I'd like to thank Grace He as well for helping me with all of my computer related needs, but mostly for taking the time to be a good friend that I could talk to in times of need. It was not always easy being the only student in a big, empty laboratory, so I really enjoyed our talks. I'd also like to thank Roy Churaman, for helping me procure the countless odds and ends that a project of this scope needs, I would not have been able to do it on my own.

I would like to thank Ryerson University, the Mechanical and Industrial Engineering department, the Natural Sciences and Engineering Research Council of Canada and Wirekörner Gmbh for their generous funding that made this project possible.

On a personal note, I'd like to thank my parents Mark and Julia and my sister Anna for always being there for me, and putting up with me; I know I can be demanding and moody sometimes. I'd like to thank my friends Vlad, Khaled, Stan and Dmitri for all the fun times we have had together, will have in the future and look forward to seeing them all at my wedding. I'd especially like to thank Dmitri, who recently completed his $\mathrm{PhD}$ for helping me with so many things around this thesis, like helping me learn LaTeX and how to make very pretty plots in MATLAB. He's the main reason it looks as good as it does.

Finally, I'd like to thank my fiancée Polly, for so many things, that it would make this thesis have a very unreasonable page count. I'll have to settle for just dedicating the whole thing to her instead. 


\section{Dedication}

To my wonderful fiancée Polly, who helped me see that I've always had the brains, heart and courage I had been looking for. 


\section{Contents}

$\begin{array}{lll}\text { Page } & \text { Pag }\end{array}$

Declaration .................................... ii

Abstract ...................... iii

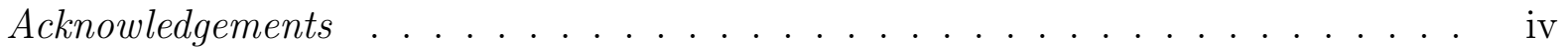

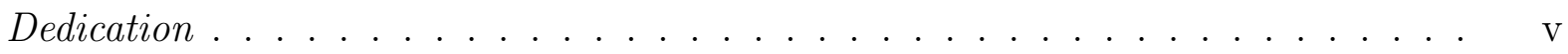

List of Figures . . . . . . . . . . . . . . . . . . . . . . . . $\mathrm{x}$

List of Tables . . . . . . . . . . . . . . . . . . . . . . xii

Nomenclature .......................... . xiii

1 Introduction $\quad 1$

1.1 Motivation . . . . . . . . . . . . . . . . . 1

1.2 Literature Review . . . . . . . . . . . . . . . . . . . 5

1.3 Scope of Thesis . . . . . . . . . . . . . . . . . . . . . 10

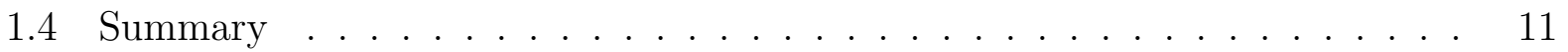

2 Theoretical Considerations $\quad 13$

2.1 States of Fluidization . . . . . . . . . . . . . . . . . . . 13

2.2 Classification of Particles . . . . . . . . . . . . . . . . . 15

2.3 Pressure Drop Through a Bed . . . . . . . . . . . . . . . . . . . . 17

2.4 Minimum Fluidization Velocity . . . . . . . . . . . . . . 19

2.4.1 Effect of Pressure and Temperature on $U_{m f} \quad \ldots \ldots$. . . . . . . . 20

2.5 Heat Transfer in Fluidized Beds . . . . . . . . . . . . . . . . . . . . 22

2.5.1 Heat Transfer Mechanisms . . . . . . . . . . . . . . . . . . . 22

2.5.2 Heat Transfer Correlation for Immersed Surfaces . . . . . . . . . . . . 25

2.6 Summary . . . . . . . . . . . . . . . . . . . . . 34

2.6.1 Fluidization . . . . . . . . . . . . . . . . . 34 
2.6 .2 Heat Transfer . . . . . . . . . . . . . . . . . . . . . . . . . . . 35

2.6 .3 Correlations . . . . . . . . . . . . . . . . . 36

3 Experimental Apparatus $\quad 37$

3.1 Furnace and Bed . . . . . . . . . . . . . . . . . . . . 37

$3.1 .1 \quad$ Fluidized Bed . . . . . . . . . . . . . . . . . . . 37

3.2 Air Delivery and Heating System . . . . . . . . . . . . . . . . 38

$3.2 .1 \quad$ Air Delivery System . . . . . . . . . . . . . . . . . 38

$3.2 .2 \quad$ Heating System . . . . . . . . . . . . . . . . . . . . . . . . . . 39

3.3 Wire Movement System . . . . . . . . . . . . . . . . . . . . . 39

3.4 Instrumentation . . . . . . . . . . . . . . . . . . . . 41

3.4.1 Temperature Measurement . . . . . . . . . . . . . . . . . . . 42

3.4 .2 Data Acquisition . . . . . . . . . . . . . . . . . . . . 45

3.4 .3 Velocity Measurement . . . . . . . . . . . . . . . . 47

3.4.4 Flow Measurement . . . . . . . . . . . . . . . . . . . . . . 47

3.5 Startup and Shutdown Procedure . . . . . . . . . . . . . . . . . . 48

$3.5 .1 \quad$ Startup . . . . . . . . . . . . . . . . . . . 48

3.5.2 Shutdown . . . . . . . . . . . . . . . . . . . . . . . 49

3.6 Limitations . . . . . . . . . . . . . . . . . . . . . . . . . . . 52

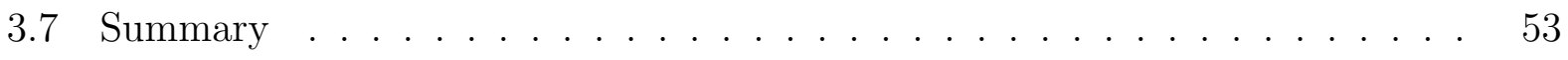

$3.7 .1 \quad$ Fluidized Bed . . . . . . . . . . . . . . . . . . . 53

3.7 .2 Wire Movement System _. . . . . . . . . . . . . . . 54

3.7.3 Instrumentation and Data Acquisition . . . . . . . . . . 54

4 Experimental Procedure $\quad 56$

4.1 Apparatus Setup . . . . . . . . . . . . . . 56

4.2 Calculation of the Heat Transfer Coefficient . . . . . . . . . . . . 57

4.2 .1 Analytical Solution . . . . . . . . . . . . . . . . . 60

$4.2 .2 \quad$ Numerical Solution . . . . . . . . . . . . . . . . . . . . . . 62 
4.2 .3 Thermometer Calibration . . . . . . . . . . . . . . . . . 64

4.3 Data Acquisition and Processing . . . . . . . . . . . . . 66

4.3 .1 Data Processing . . . . . . . . . . . . . . . . . . 67

4.4 Tests Conducted . . . . . . . . . . . . . . . . . . . . 68

$4.4 .1 \mathrm{U}_{m f}$ Comparisons . . . . . . . . . . . . . . . 68

4.4 .2 Experimental Matrix . . . . . . . . . . . . . . . . . 68

$\begin{array}{lll}5 & \text { Results and Discussion } & \mathbf{7 0}\end{array}$

5.1 Properties of $\mathrm{Al}_{2} \mathrm{O}_{3}$ Particles . . . . . . . . . . . . . . 70

5.2 Thermometer Calibration and Testing . . . . . . . . . . 73

5.2 .1 Calibration . . . . . . . . . . . . . . . . . . 73

5.2 .2 Frictional Heating . . . . . . . . . . . . . . . . . . 77

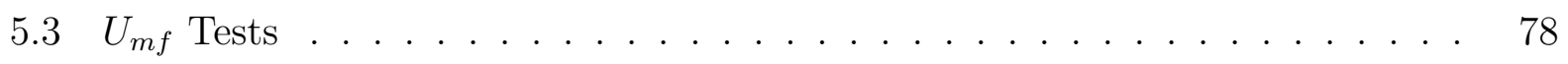

5.460 Grit Tests . . . . . . . . . . . . . . . . . 78

$5.5 \quad 70-80$ Grit Mix Tests . . . . . . . . . . . . . . . . . . 80

5.6 Discussion of Results . . . . . . . . . . . . . . . . . 81

5.6.1 Comparison of 60 and $70-80$ Grit Mix . . . . . . . . . . 82

5.6 .2 Comparison to Existing Correlations _ . . . . . . . . . . 85

$\begin{array}{lll}6 & \text { Error Assessment } & 95\end{array}$

6.1 Introduction . . . . . . . . . . . . . . . . . . . 95

6.2 Air Flow Uncertainties . . . . . . . . . . . . . . . . . . . . . . 95

6.3 Heat Transfer Uncertainties . . . . . . . . . . . . . . . . . . . 97

6.3 .1 Wire Speed Uncertainties . . . . . . . . . . . . . . . . . 101

6.4 Non-Quantifiable Uncertainties . . . . . . . . . . . . . . . . . . . 101

7 Conclusions and Recommendations 103

7.1 Conclusions . . . . . . . . . . . . . . . . . . . . . . 103

7.2 Recommendations . . . . . . . . . . . . . . . 106

7.2 .1 Apparatus . . . . . . . . . . . . . . . 106

viii 
7.2 .2 Studies . . . . . . . . . . . . . . . . 107

$\begin{array}{ll}\text { References } & 112\end{array}$

$\begin{array}{ll}\text { Appendices } & 113\end{array}$

$\begin{array}{lr}\text { A List of Publications } & 114\end{array}$

B Matlab Scripts and Functions $\quad 115$ 


\section{List of Figures}

1.1 Wire drawing and heat treatment process . . . . . . . . . . . . 3

2.1 Regimes of fluidization . . . . . . . . . . . . . . . . . 14

2.2 Geldart's classification of powders . . . . . . . . . . . . . 16

2.3 Minimum fluidization velocity at different temperatures and pressures . . . . 21

2.4 Modes of heat transfer in gas fluidized beds . . . . . . . . . . . . 23

2.5 Effects of air velocity and wire speed on particle convection . . . . . . . . 25

2.6 Sphericity vs bed voidage . . . . . . . . . . . . . . . . . 27

2.7 Friedman's experimental data vs existing correlations . . . . . . . . . . . 29

3.1 Cross section of fluidized bed . . . . . . . . . . . . . . . . 38

3.2 Air delivery and heating system . . . . . . . . . . . . . . . 40

3.3 Pulling side of wire movement system . . . . . . . . . . . . . . . . 41

3.4 Feeding side of wire movement system . . . . . . . . . . . . . . . . 42

3.5 Top and section view of apparatus . . . . . . . . . . . . . . 43

3.6 Isometric view of apparatus . . . . . . . . . . . . . . . . . . 44

3.7 DT9828 data acquisition unit and connections . . . . . . . . . . . . 46

3.8 System startup procedure . . . . . . . . . . . . . . . . . 50

3.9 Wiring of various systems . . . . . . . . . . . . . . . . 51

4.1 Energy balance on a control volume of wire . . . . . . . . . . . . 58

4.2 Numerical vs analytical solution . . . . . . . . . . . . . . . . . 63

4.3 Calibration fixture and setup . . . . . . . . . . . . . 65

5.1 Size distribution of 60 grit particles . . . . . . . . . . . . . . 72

5.2 Size distribution of $70 \& 80$ grit particle mix . . . . . . . . . . . . . . 72

5.3 FEHT model of calibration apparatus . . . . . . . . . . . . . . 75 


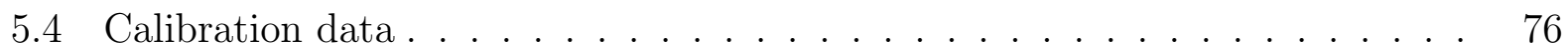

5.5 Variations in inlet temperature vs wire speed . . . . . . . . . . 77

5.6 Nusselt numbers at various fluidizing velocities and wire speeds _ . . . . . 79

5.7 Nusselt numbers at various wire speeds in 60 grit sand $\ldots \ldots$. . . . . 80

5.8 Nusselt numbers at various wire speeds in 70 and 80 grit sand . . . . . . 81

5.9 Nusselt numbers at various wire speeds in both sets of particles . . . . . . 83

$5.10 \mathrm{~h}$ and Nusselt number for 60 grit sand . . . . . . . . . . . . 85

$5.11 \mathrm{~h}$ and Nusselt number for a mix of 70 and 80 grit sand $\ldots \ldots . \ldots 86$

5.12 Experimental data compared to existing correlations . . . . . . . . . 87

5.13 Equation 5.5 compared to experimental data and correlations $\ldots . . . .89$

5.14 Equation 5.5 vs experimental data . . . . . . . . . . . . . . . 90

5.15 Equation 5.9 vs experimental data . . . . . . . . . . . . . . . . . 92

5.16 Equation 5.9 compared to Equation 5.5 and experimental data . . . . . . 93

6.1 Outlet temperature vs wire thermal conductivity . . . . . . . . . . . 98 


\section{List of Tables}

1.1 Correlations for predicting the Nusselt number . . . . . . . . . . 8

4.1 Tests at various values of $U_{m f}$ and Wire Speed . . . . . . . . . . . . . 68

4.2 Test Matrix. All tests conducted at a Fluidizing rate of $3 \times U_{m f} \ldots \ldots 9$

5.1 Properties of 8 and 60 grit particles . . . . . . . . . . . 70

5.2 Properties of $70 \& 80$ grit mix particles . . . . . . . . . . . 73 


\section{Nomenclature}

\begin{tabular}{|c|c|}
\hline$\alpha_{c}$ & Thermal Diffusivity of the cluster $\left(\frac{m^{2}}{s}\right)$ \\
\hline$\alpha_{w}$ & Thermal Diffusivity of the wire $\left(\frac{m^{2}}{s}\right)$ \\
\hline$\delta$ & Gas Gap Thickness $(m)$ \\
\hline$\Delta_{p}$ & Pressure drop across the bed $(P a)$ \\
\hline$\mu_{g}$ & Viscosity of the gas $\left(\frac{\mathrm{kg}}{\mathrm{sm}}\right)$ \\
\hline $\bar{h}_{c}$ & Overall Heat Transfer Coefficient $\left(\frac{W}{m^{2} K}\right)$ \\
\hline $\bar{h}_{c c_{t<t_{0}}}$ & Partial Penetration Heat Transfer Coefficient $\left(\frac{W}{m^{2} K}\right)$ \\
\hline $\bar{h}_{c c_{t>t_{0}}}$ & Full Penetration Heat Transfer Coefficient $\left(\frac{W}{m^{2} K}\right)$ \\
\hline $\bar{h}_{c c}$ & Heat Transfer Coefficient due to Particle Convection $\left(\frac{W}{m^{2} K}\right)$ \\
\hline $\bar{h}_{g c}$ & Heat Transfer Coefficient due to Gas Convection $\left(\frac{W}{m^{2} K}\right)$ \\
\hline $\bar{h}_{w \max }$ & Maximum Heat Transfer Coefficient $\left(\frac{W}{m^{2} K}\right)$ \\
\hline $\bar{p}$ & Average Absolute Pressure in the Bed $(P a)$ \\
\hline$\phi_{s}$ & Particle Sphericity \\
\hline$\rho_{b}$ & Bulk Density of bed $\left(\frac{\mathrm{kg}}{\mathrm{m}^{3}}\right)$ \\
\hline$\rho_{c}$ & Density of cluster $\left(\frac{\mathrm{kg}}{\mathrm{m}^{3}}\right)$ \\
\hline$\rho_{g}$ & Gas Density $\left(\frac{\mathrm{kg}}{\mathrm{m}^{3}}\right)$ \\
\hline
\end{tabular}



$\rho_{s}$
Particle Density $\left(\frac{\mathrm{kg}}{\mathrm{m}^{3}}\right)$
$\rho_{w}$
Wire Density $\left(\frac{\mathrm{kg}}{\mathrm{m}^{3}}\right)$
$\tau$
Particle or cluster residence time at the surface $(s)$
$\Theta(x)$
Temperature difference between the bed and wire temperatures = $T(x)-T_{\infty},\left({ }^{\circ} \mathrm{C}\right)$
$\Theta_{i n}$
Temperature difference between the bed and wire inlet temperatures $=T_{\text {in }}-T_{\infty},\left({ }^{\circ} \mathrm{C}\right)$
$\varepsilon$
Average Packed Bed Voidage
$\varepsilon$
Bed Voidage
$\varepsilon_{c}$
Cluster Voidage
$\varepsilon_{m f}$
Minimum Bed Voidage at $U_{m f}$
$A_{b}$
Effective Bed Area $\left(m^{2}\right)$
$A_{c}$
Wire cross sectional area $\left(m^{2}\right)$
Ar
Archimedes number $=\frac{\rho_{g} d_{p}^{3}\left(\rho_{s}-\rho_{g}\right) g}{\mu_{g}^{2}}$
$B i$
Biot number $=\frac{h_{g} d_{c}}{k_{c}}$
$C_{c}$
Specific Heat Capacity of the cluster $\left(\frac{J}{k g K}\right)$
$C_{p, g}$
Specific Heat Capacity of the gas $\left(\frac{J}{k g K}\right)$
$C_{p, s}$
Specific Heat Capacity of the particles $\left(\frac{J}{k g K}\right)$ 


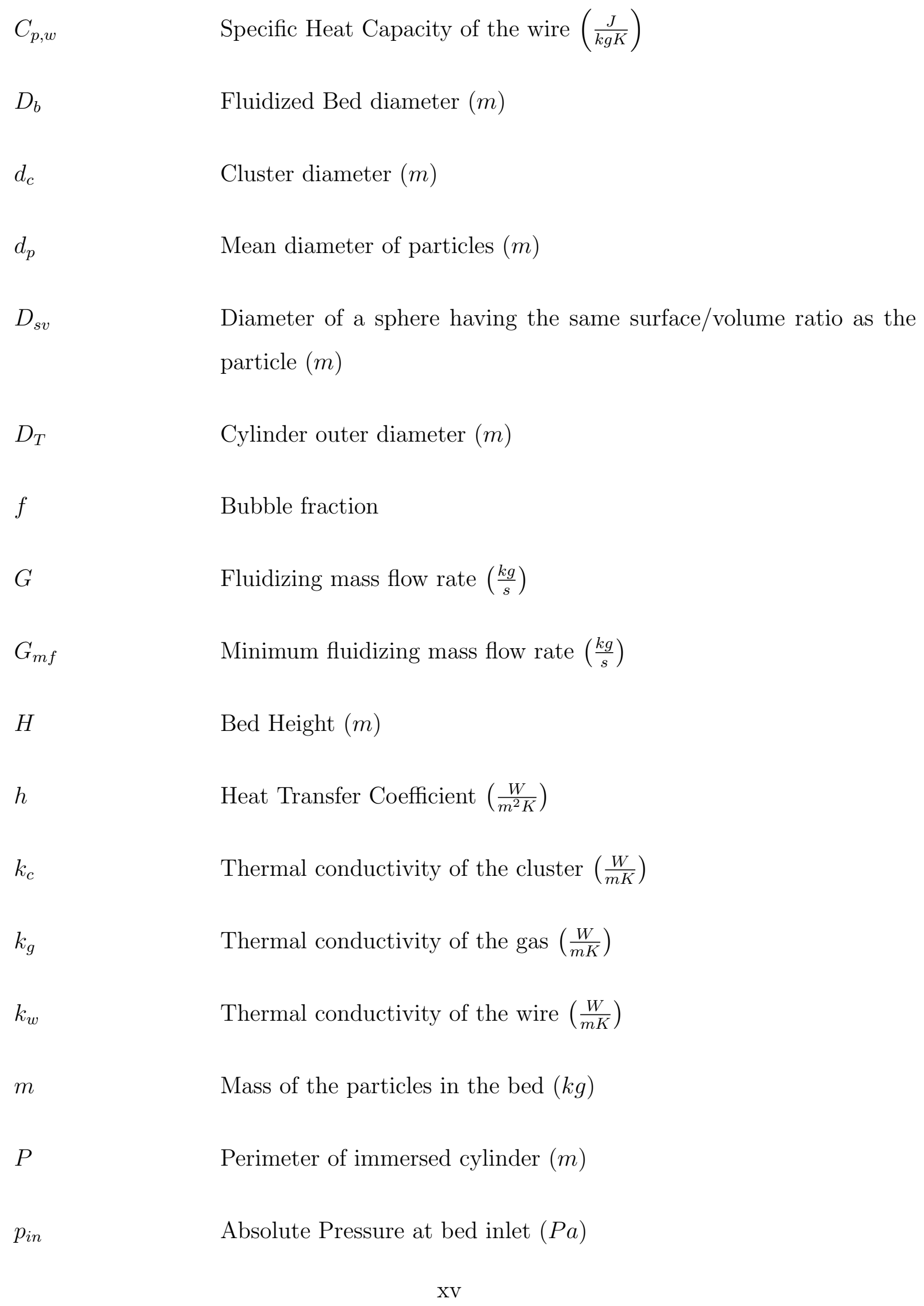




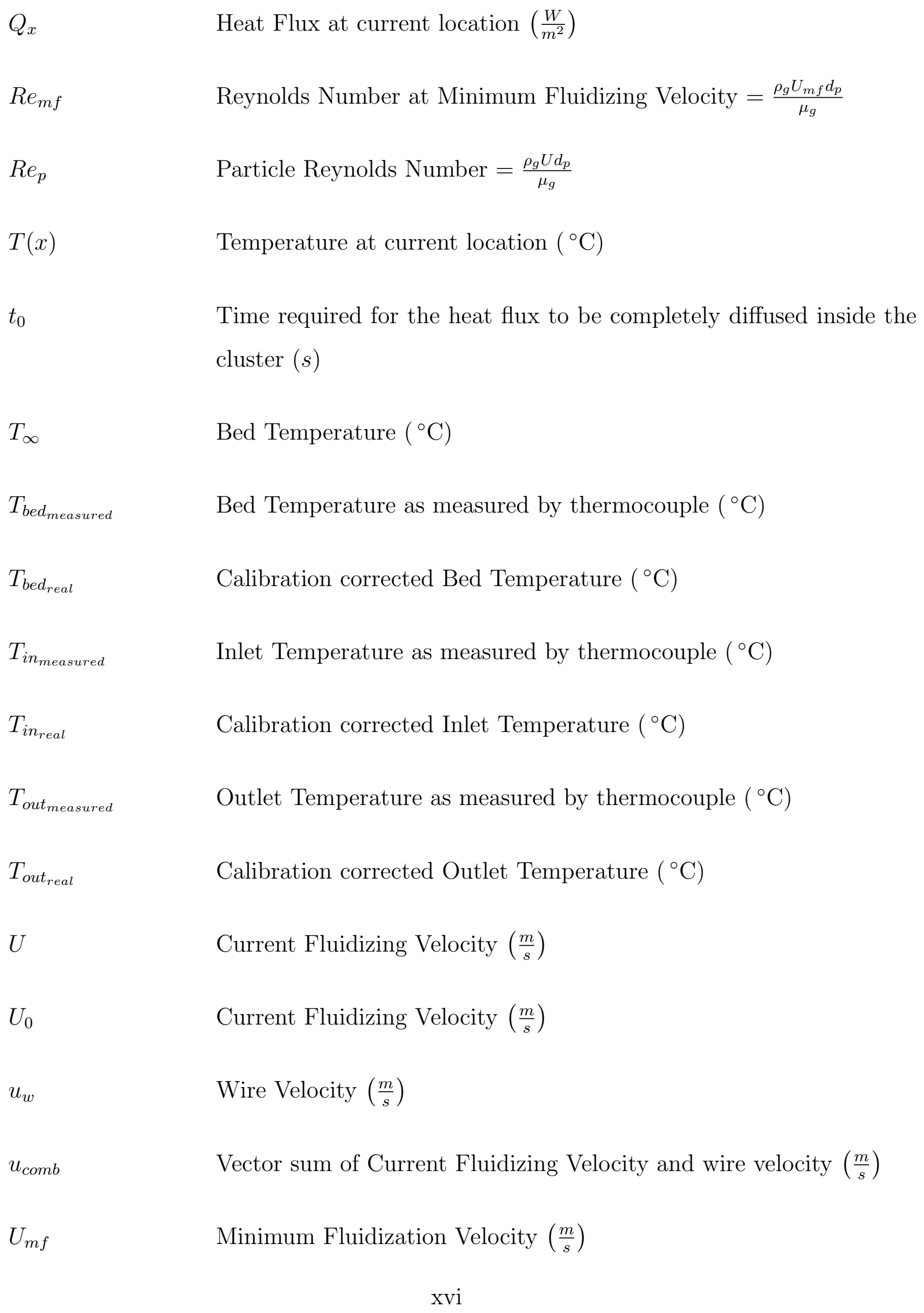


MFC

MMC

MPD

$\mathrm{Nu}$

$\mathrm{Nu}_{\text {mean }}$ moving

$\mathrm{Nu}_{\text {mean }}$ static

$\operatorname{Pr}$
Modified Friedman Correlation

Modified Masoumifard Correlation

Mean Particle Diameter

Nusselt Number $=\frac{h D_{t}}{k_{g}}$

Mean Nusselt Number for a moving wire

Mean Nusselt Number for a static wire

Prandtl Number $=\frac{C_{p, g} \mu_{g}}{k_{g}}$ 


\section{CHAPTER 1}

\section{Introduction}

\subsection{Motivation}

Steel wire has permeated many parts of modern society. From the nails we use for building, the wire hangers in our closets, high strength wire ropes, to the wire mesh fences we see surrounding many properties; wire has found a use in nearly all we do. This wire is manufactured through a process called wire drawing, which involves pulling a large diameter rod through several dies of decreasing size to get it to the desired diameter. This causes work hardening, making the wire stiff, rigid, and incapable of being shaped into the various forms we need.

Heat treatment provides a solution to this problem, making the wire stronger and more ductile so that it can be formed as needed. There are two methods of heat treating wire: batch and continuous processes. In a batch process, a large quantity of wire is loaded into the furnace and then sealed for heat treatment, whereas in a continuous process the wire is fed through the furnace with many strands of wire running in parallel. Continuous wire processing is capable of heat treating tens of thousands of pounds of wire each hour. For the purpose of this study, only continuous processes will be discussed.

In addition, there are also two main types of steel wire: high carbon steel and low carbon steel. There are important differences in material properties between high and low carbon steel wire, and the heat treatment process for these two types of wire is consequently different. Low carbon steel wire is softer and more ductile, allowing it to be formed more easily. The wire drawing process, however, makes it quite brittle, so to restore its malleable nature it is commonly heat treated by immersing it into a molten lead furnace running at $730{ }^{\circ} \mathrm{C}$ until the wire reaches a temperature of $710^{\circ} \mathrm{C}$. The duration of this process and the cooling of 


\subsection{Motivation}

the wire is not critical, so many options are available when it comes to furnaces that can be used. Upon completion, the wire can be shaped into common products such as wire hangers, meshes and many other objects.

High carbon steel wire, on the other hand, is very strong, and typically used for products like springs, wire ropes and in construction materials, such as rebar in pre-stressed concrete. Since most of the applications rely on its high strength, the heat treatment of high carbon steel wire is more critical. In a process called patenting, which is used for many types of high carbon steel wire, the wire is heated to over $800^{\circ} \mathrm{C}$ and then rapidly quenched to $480^{\circ} \mathrm{C}$ followed by a soak at $480^{\circ} \mathrm{C}$. Patenting is usually done in two different furnaces, the first being a direct fired furnace operating at $1050^{\circ} \mathrm{C}$. This type of furnace relies on radiation and air convection to achieve its goals, and as such has a fairly low heat transfer rate which means it is not very energy efficient. Because of the low heat transfer rates attained by direct fired furnaces, they are also quite long, taking up plenty of space that could be used for other purposes. The quench and soak are usually performed in a molten lead bath, wherein rollers immerse the wire in the molten lead. A schematic of the heat treatment and the preceding wire drawing process can be seen in Figure 1.1. Molten lead provides very high heat transfer rates, on the order of $1200-1800 \mathrm{~W} / \mathrm{m}^{2} \mathrm{~K}$ but poses a hazard to workers and the environment from the fumes that it emits in its liquid state. Many jurisdictions have completely banned the use of molten lead due to these concerns.

To alleviate the bulkiness and poor efficiency of direct fired furnaces, as well as the environmental hazards presented by a molten lead bath, a fluidized bed furnace can be used. Fluidized bed furnaces are not a recent invention. In fact, they have been around since the mid-1950s in a variety of application such as coal gasification, wastewater treatment and even as a catalyst for certain chemical processes. A fluidized bed consists primarily of many solid particles, a vessel or container for the aforementioned particles, a blower to provide pressurized gas and a porous distributor to support the particles and distribute the pressurized gas. A fluidized bed can become a fluidized bed furnace if a heating method is added to it, such as the combustion of natural gas within the fluidized bed, or an electric heater. Due to the very high surface area of the particles in a fluidized bed furnace, they are capable of high heat transfer rates. They also experience a high degree of solids mixing, so the temperature 


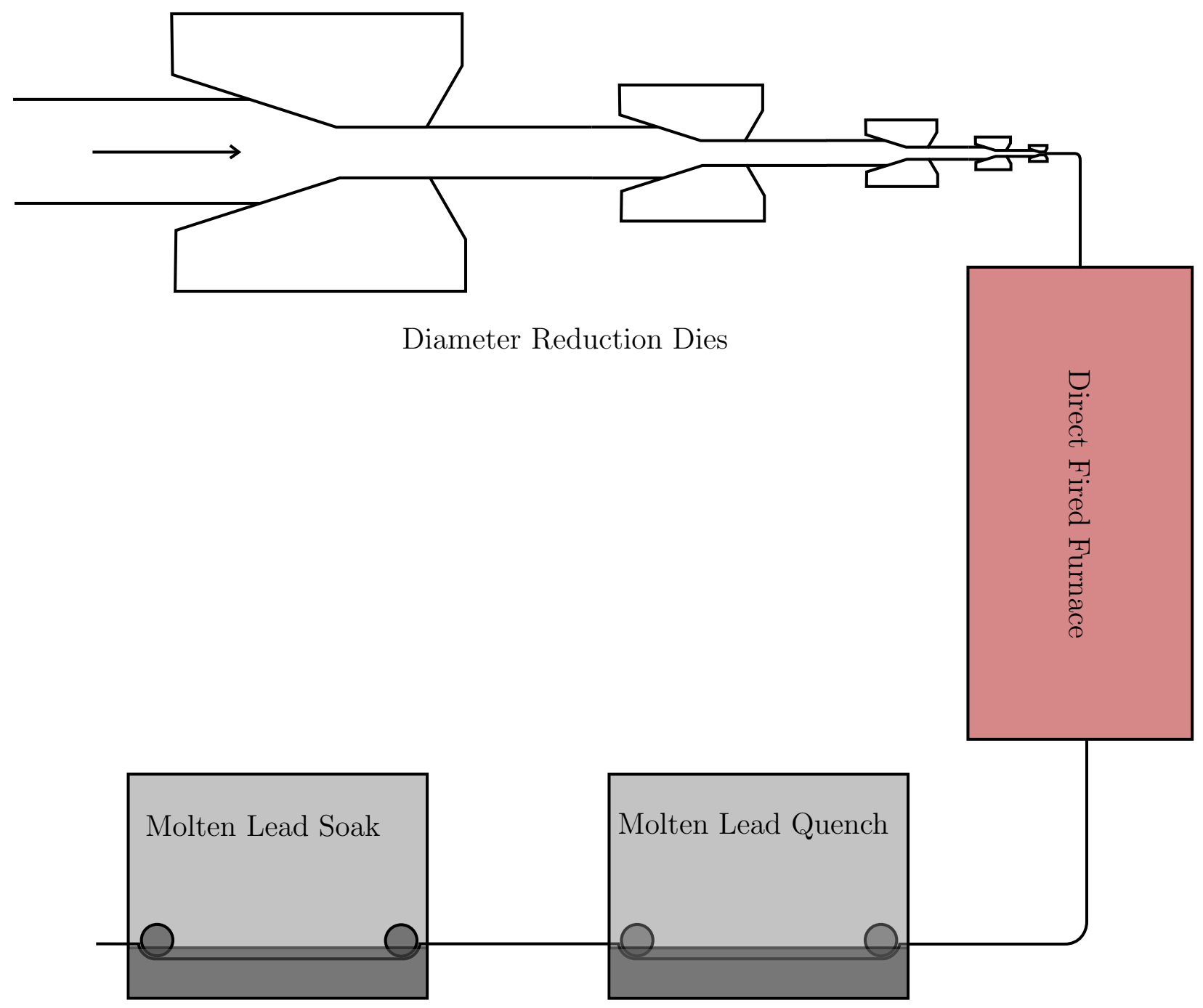

Figure 1.1: Schematic of wire drawing and heat treatment process

gradients are quite low (Kunii and Levenspiel, 1977). Thus, fluidized bed furnaces offer some great advantages, such as high heat transfer rate and high temperature uniformity which is desirable in many instances. Furthermore, since the fluidization media itself is not harmful to the environment, the only potential source of pollution or hazard comes from the heat generation. There are some difficulties that stand in the way of adoption of fluidized bed furnaces for the heat treatment of high carbon steel wire however, and that is the process' requirement for precise heating and cooling rates as well as temperatures. Currently, there is insufficient understanding of the heat transfer rates to small diameter wire $(1-8 \mathrm{~mm})$ that 


\subsection{Motivation}

is being heated in a continuous process inside a fluidized bed.

There were many studies conducted in the past by the power generation industry into heat transfer to boiler tubes of diameters between $25 \mathrm{~mm}$ and $50 \mathrm{~mm}$ immersed in a fluidized bed furnace (Botterill and Desai (1972), Grewal and Saxena (1980), Saxena (1989)). Work on small diameter cylinders such as wire in the 1-6 $\mathrm{mm}$ range has been sparse, however, and extrapolating from the correlations found for the larger diameter boiler tubes gives contradictory results which vary based on whose correlation is used (Friedman et al., 2006). There have been some workers in recent years who have studied small diameter wire, but the experiments have all been carried out on static wire (Friedman et al. (2006), Masoumifard et al. (2008)), despite the fact that the correlations developed are meant for use in a continuous process, in which the wire is moving through the fluidized bed. Due to the difficulty involved with constructing a laboratory scale wire drawing system, such studies have submerged the wire into a fluidized bed and kept it stationary while using various probes to determine the heat transfer rate into the wire. This process may not accurately represent the continuous wire heat treatment process, so it is possible that the correlations developed in this manner would not adequately predict the heat transferred into the wire. There is reason to believe that the wire's motion will affect the heat transfer rate from the bed to the wire, the details of which will be discussed further in the next chapter on theoretical concerns.

For this purpose, the first laboratory scale wire movement system has been designed, developed and installed at Ryerson University's Fluidized Bed Heat Transfer Laboratory. The system includes multiple spools and a wire speed regulation system as well as wire straightening to ensure good data accuracy and repeatability. The wire is fed through a rectangular air fluidized bed filled with fine grit aluminum oxide sand and heated by an electrically powered heater. The temperatures at the wire inlet and outlet are obtained by specialty moving wire thermocouples, while an immersion type probe measures the bed temperature. With this data the heat transfer into the wire can be determined and a correlation can be obtained that will give deeper insight into how different factors affect the heat transfer, allowing fluidized beds to be more precisely designed for the heat treatment of high carbon steel wire. 


\subsection{Literature Review}

\subsection{Literature Review}

Heat transfer to immersed surfaces in a fluidized bed furnace is a topic that has been studied extensively over the years by quite a number of workers. In addition to the founding work of Mickley and Fairbanks (1955), Zabrodsky (1966), Geldart (1973) and Botterill (1975), there have been numerous attempts to develop correlations for heat transfer to immersed surfaces, the bulk of which are catalogued in Saxena (1989). More recent investigations by Friedman et al. (2006) and Masoumifard et al. (2008) have shown that the correlations developed for medium and large diameter tubes do not hold for small diameter wire. This section will detail the contributions made by previous workers that have shaped the field into what it is today.

Berg and Baskakov (1966) conducted one of the earliest studies of small diameter cylinders in a gas fluidized bed. Their work focused on vertical immersed cylinders of 3, 6, 10, 19.7 and $19.8 \mathrm{~mm}$ rotating about their axis. All of the aforementioned cylinders were copper, except for the $19.7 \mathrm{~mm}$ which was made of porcelain. The bed used in their experiments was a $200 \mathrm{~mm}$ diameter, $800 \mathrm{~mm}$ tall plexiglass tube, with a steel gas distributor. Particles used in the bed were electrical corundum belonging to Geldart Group A and B, of 60, 120 and $320 \mu \mathrm{m}$, filled to a height of $360 \mathrm{~mm}$. They found a strong dependence on cylinder diameter for heat transfer to cylinders between 3-10 $\mathrm{mm}$, but it had a reduced impact in the case of the larger diameter cylinders. Heat transfer is also strongly dependent on particle diameter. They also noted that the rate of rotation of the cylinder led to an increase in heat transfer rate at higher fluidizing velocities. The applicability of their work to horizontal moving wire is highly questionable however, as the geometry of the experiment and the nature of the motion are drastically different.

Botterill and Desai (1972) studied the factors which affect heat transfer in gas fluidized beds. They utilized an annular shaped, stirred packed bed with the stirrer blades near the heat transfer surfaces in order to be able to control the rate of packet exchange near the heat transfer surfaces. They discovered that the stirrers were not sufficient as a method of controlling packet exchange rates and packet residence times on the heat transfer surfaces, 


\subsection{Literature Review}

so they performed additional experiments with a hopper that allowed the particles to flow down a column past the heat exchange surface. While this system was not a fluidized bed, they postulated that it accurately imitated the conditions inside one. They noticed that there was a strong connection between particle residence time and heat transfer, and that short residence times and high rates of packet exchange led to greatly increased heat transfer coefficients. They also concluded that the heat transfer rate was more dependent on the thermal conductivity of the gas than on the thermal conductivity of the particles, and noted that this effect is due to the minimal direct contact between the particles and the heat transfer surfaces. The bulk of the heat transfer between the particles and the surface would occur in the thin layer of gas between the particle and surface, commonly referred to as the gas lens. Many of their conclusions were supported in future works and are now largely accepted as correct. However, they were not able to develop a usable model for predicting heat transfer inside a fluidized bed furnace.

Martin (1984) developed a mechanistic model of fluid bed heat transfer based on a single particle for the particle convective heat transfer. The gas convection and radiation components were based on commonly accepted correlations found by previous workers. This model was then evaluated by checking the effects of various parameters on the heat transfer coefficient. Particle size, size distribution, physical properties of the particles and gas, temperature, pressure and bed expansion were all investigated and the model was found to be in good agreement with the data of Wunder (Martin, 1984). The relationship between the diameter of the immersed object and heat transfer was not investigated however, and most of the tubes used in the experiments were of relatively large diameter $(30-40 \mathrm{~mm})$.

Grewal and Saxena (1980) performed a study of the heat transfer coefficient in a gas fluidized bed with dimensions of $30.5 \mathrm{~cm} \times 30.5 \mathrm{~cm}$ filled with various particles, such as glass beads, dolomite, sand, silicone carbide and alumina. Three different distributors were utilized, two perforated plates with an open area of $37.5 \%$ and $7.7 \%$ respectively, and a bubble cap distributor with an open area of $0.2 \%$. Three different electrically heated tubes were tested, with diameters of $12.7 \mathrm{~mm}, 19.05 \mathrm{~mm}$ and $28.6 \mathrm{~mm}$ respectively. They found that the heat transfer rate would initially increase with increasing fluidizing velocity, but reached a maximum value and would decrease with additional velocity. Their reasoning was that the 


\subsection{Literature Review}

nature of the flow inside the bed would change the contributions of the particle and gas convection and that an optimal value exists at some intermediate point. Similar to other workers, they noted that an increase in particle diameter led to decreased heat transfer rates, as the heat transfer mechanism would rely more on gas convection in those cases. They also concluded that bed height and the thermal conductivity of the heat transfer surface did not play a major role. Additionally, their work supports findings by earlier researchers that heat transfer rates decrease as the diameter of the heat transfer surface is increased. Their study of different distributors determined that the nature of the distributor plays a greater role at lower fluid velocities but does not heavily influence heat transfer at greater velocities. They also went on to develop a correlation for heat transfer to immersed cylinders in a gas fluidized bed, and compared it to seven previous correlations by other workers over a wide range of experimental data. It is similar to that of Vreedenberg (1958), but includes an extra dimensionless term to account for volumetric heat capacity of the solid particles, the tube diameter and the thermal conductivity of the gas. This correlation was able to predict nearly all of the previous experimental data within a $\pm 25 \%$ range.

Grewal and Saxena (1981) went on to propose a correlation for maximum heat transfer coefficient between a horizontal immersed tube in a fluidized bed by expanding on their previous work. They managed to predict nearly all of the data within a narrower range of $\pm 20 \%$ in this case. Grewal (1982) later noticed that this correlation fails at Archimedes numbers less than 300 when compared to the data of a study performed by the Aerojet Energy Conversion Company (1980) and his proposed correction managed to predict all of the Aerojet data within a 15\% margin.

Saxena (1989) went on to present a comprehensive summary of previous work on the topic of heat transfer between horizontal tubes immersed in gas fluidized beds which can be seen in Table 1.1. He noted that the majority of the correlations have limited applicability as they are restricted to certain operating conditions. The correlation by Grewal and Saxena (1980) was the only one capable of predicting a large amount of experimental data within a range of $\pm 25 \%$.

An important issue with these correlations, is that there is no agreement of the dependence 


\subsection{Literature Review}

Table 1.1: Correlations for predicting the Nusselt number (Adapted from Saxena (1989))

\begin{tabular}{|c|c|c|}
\hline Predicted Correlation & Reference & $\mathrm{Nu} \propto \mathrm{D}_{t}^{n}$ \\
\hline $\mathrm{Nu}=420\left[\left(\frac{G D_{T} \rho_{s}}{\rho_{g} \mu_{g}}\right)\left(\frac{\mu_{g}^{2}}{d_{p}^{3} \rho_{s}^{2} g}\right)\right]^{0.3} \operatorname{Pr}^{0.3}$ & $\begin{array}{l}\text { Vreedenberg } \\
\text { (1958) }\end{array}$ & $\mathrm{Nu} \propto D_{t}^{0.3}$ \\
\hline $\mathrm{Nu}=14\left(\frac{G}{G_{m f}}\right)^{\frac{1}{3}} \operatorname{Pr}^{\frac{1}{3}}\left(\frac{D_{T}}{d_{p}}\right)^{\frac{2}{3}}$ & $\begin{array}{l}\text { Petrie et al. } \\
(1967)\end{array}$ & $\mathrm{Nu} \propto D_{t}^{0.67}$ \\
\hline $\mathrm{Nu}=5.76(1-\varepsilon)\left(\frac{G d_{p}}{\mu_{g} \varepsilon}\right)^{0.34} \operatorname{Pr}^{0.33}\left(\frac{H}{D_{b}}\right)^{0.16}\left(\frac{D_{T}}{d_{p}}\right)$ & $\begin{array}{l}\text { Zabrodsky } \\
\text { (1966) }\end{array}$ & $\mathrm{Nu} \propto D_{t}^{1}$ \\
\hline $\mathrm{Nu}=900(1-\varepsilon)\left[\left(\frac{G D_{T} \rho_{s}}{\rho_{g} \mu_{g}}\right)\left(\frac{\mu_{g}^{2}}{d_{p}^{3} \rho_{s}^{2} g}\right)\right]^{0.326} \operatorname{Pr}^{0.3}$ & $\begin{array}{l}\text { Andeen and } \\
\text { Glicksman } \\
(1976)\end{array}$ & $\mathrm{Nu} \propto D_{t}^{0.33}$ \\
\hline $\begin{aligned} \mathrm{Nu}=47(1-\varepsilon) & {\left[\left(\frac{G D_{T} \rho_{s}}{\rho_{g} \mu_{g}}\right)\left(\frac{\mu_{g}^{2}}{d_{p}^{3} \rho_{s}^{2} g}\right)\right]^{0.325} \operatorname{Pr}^{0.3} } \\
& {\left[\frac{\rho_{s} C_{p, s} D_{T}^{\frac{3}{2}} g^{\frac{1}{2}}}{k_{g}}\right]^{0.23} }\end{aligned}$ & $\begin{array}{l}\text { Grewal and Sax- } \\
\text { ena (1980) }\end{array}$ & $\mathrm{Nu} \propto D_{t}^{0.67}$ \\
\hline
\end{tabular}

of Nusselt number on the diameter of the immersed surface as can be seen in Table 1.1. These correlations were meant for tubes in the $25-75 \mathrm{~mm}$ range and when applying them to wire of diameter between 1-6 $\mathrm{mm}$, it becomes obvious that they would lead to drastically different predictions for heat transfer.

Friedman et al. (2006) studied heat transfer to small cylinders ranging from $1.3 \mathrm{~mm}$ to 9.5 $\mathrm{mm}$ in order to simulate the conditions in a typical wire heat treatment fluidized bed furnace. Two fluidized beds were used, a laboratory scale unit with a diameter of $300 \mathrm{~mm}$ and a pilot scale unit of $500 \mathrm{~mm}$ by $1000 \mathrm{~mm}$. Both were used at atmospheric pressure, but the pilot scale unit was used for high temperature tests up to $750{ }^{\circ} \mathrm{C}$ while the laboratory scale unit only conducted ambient temperature testing. The data was collected using thermocouples welded to the inside of the tubes at each end as well as the mid-point and one thermocouple $30 \mathrm{~mm}$ away to measure the bed temperature. Their results showed that 


\subsection{Literature Review}

all of the past correlations that were made for larger diameter cylinders grossly over-predict the heat transfer from small diameter cylinders. They also found that the heat transfer remains nearly constant after approximately $2.5 \times \frac{G}{G_{m f}}$, whereas previous correlations predict a steady increase. Due to these findings, $G$ (fluidizing gas mass flux) was eliminated from the relationship as well as $\varepsilon$ (bed voidage) due to its dependence on G. Their correlation found the mean Nusselt number to be proportional to Archimedes number and the ratio of tube over particle diameter $N u_{\text {mean }}=C A r^{n} \frac{d_{t}}{d_{p}}$ with $C=1.35$ and $n=0.15$. This simple correlation is meant for engineering application, but it has proven effective at predicting heat transfer rates to small immersed cylinders.

Masoumifard et al. (2008) studied the influence of axial position, particle diameter and superficial gas velocity on heat transfer from an $8 \mathrm{~mm}$ cylinder in a fluidized bed. They used a bed of $150 \mathrm{~mm}$ diameter and $2000 \mathrm{~mm}$ height and sand particles with a mean diameter of 280,490 and $750 \mu \mathrm{m}$. Superficial air velocity was varied from $U_{m f}$ (minimum fluidizing velocity) to $1.20 \mathrm{~m} / \mathrm{s}$. As $U_{m f}$ is dependent on particle diameter, the range of multiples of $U_{m f}$ tested for each particle was different. The measurements were taken using a thermocouple located on the center of the tube and an RTD sensor located $50 \mathrm{~mm}$ away to measure the bed temperature. Their results showed that heat transfer is independent of axial position in the bed and that heat transfer was inversely proportional to particle diameter. For superficial air velocity, there was a rapid increase in heat transfer up to a maximum and then a gradual decrease as the velocity continues to be increased. This agrees well with findings from other workers. They also developed a correlation to predict the heat transfer to immersed cylinders. It was based on a cluster renewal method and showed that heat transfer rates were highly dependent on $\tau$, the contact time between a cluster of particles and the immersed surface.

Masoumifard et al. (2010) sought to predict the maximum heat transfer coefficient to a small diameter $(8 \mathrm{~mm})$ horizontal tube immersed in a gas fluidized bed as well as the superficial air velocity at which it occurs using the same model they had developed in their previous work. They determined a time, $t_{0}$, which is the duration of time required for the heat flux to completely diffuse inside the cluster. The maximum heat transfer rate would occur when the contact time, $\tau$, would be equal to $t_{0}$. They compared their correlation with 


\subsection{Scope of Thesis}

the experimental data as well as experimental data from previous studies. Their correlation managed to predict the optimal superficial air velocity for all of the data within a $20 \%$ margin of error, but some of the values for maximum heat transfer lay outside of the predictions. The discrepancy was attributed to different fluidization characteristics due to variances in bed geometry. Both this and their previous work illustrate the importance of the contact time between the cluster of particles and the immersed surface.

While recent work on small diameter wire has managed to produce useful correlations, there is a lot of concern as to whether or not a correlation developed for static wire can accurately predict the heat transfer rates to a moving wire in a fluidized bed. Friedman et al. (2006) developed a simple engineering correlation that is accurate for small diameter cylinders, but is based on a static wire. Masoumifard et al. (2008) constructed a more complex model, that is capable of predicting the heat transfer rates over a wider range of applications, but it is highly dependent on the cluster and immersed surface contact time, $\tau$, which will change as soon as the immersed surface undergoes any sort of motion. Berg and Baskakov (1966) showed this dependence on movement of the immersed surface clearly, even though their study looked at rotating motion, not horizontal. From these previous studies it can be seen that when there is motion of the immersed surface, there will be some influence on heat transfer rates. The present study was conducted to gain a better understanding of these effects.

\subsection{Scope of Thesis}

The combination of environmental pressure on industry to move away from molten lead and the lack of previous work on the effects of longitudinal wire movement on heat transfer to the immersed wire have created a need to fill that knowledge gap. This work has several goals:

- To develop an experimental apparatus to feed a wire at a constant, controlled speed through a fluidized bed furnace 
- To develop a model capable of determining the average heat transfer coefficient and Nusselt number based on available parameters such as inlet, outlet and bed temperatures, as well as wire speed

- To measure the effect of wire speed on the average heat transfer coefficient and Nusselt number

- To produce a simple engineering correlation that can be used to predict the Nusselt number for a moving wire immersed in a fluidized bed furnace

\subsection{Summary}

Wire is a very useful product, but its traditional production method causes work hardening and many applications of wire require that it undergo heat treatment prior to forming or use. For low carbon steel wire, the process is straightforward, but high carbon steel wire requires precise control of heating and cooling rates and has typically relied on molten lead baths to achieve this. These are very harmful to both operator and environment, and a more benign alternative is desired. Fluidized beds offer one such alternative, but our current understanding of heat transfer rates inside them is not sufficient to be able to achieve the precise heating and cooling rates needed for the heat treatment of high carbon steel.

There have been many studies of heat transfer to immersed surfaces in fluidized beds, from workers such as Berg and Baskakov (1966), Botterill and Desai (1972) and Grewal and Saxena (1980). The bulk of these however, were intended for the power generation industry and the correlations that were developed are only applicable to large diameter tubes, such as the boiler tubes used in power generation. More recent work by Friedman et al. (2006) and Masoumifard et al. (2008) has shown that correlations developed for large diameter tubes, do not accurately predict the heat transfer to wire sized objects.

These studies resulted in correlations that accurately predict the heat transfer to wire sized objects (1-8 $\mathrm{mm}$ in diameter), however these, and all previous studies have been performed 
on static immersed objects, where as typical industry practice is to have the wire fed longitudinally through the furnace. For this purpose an apparatus was constructed to feed a wire through a fluidized bed and measure the resulting heat transfer. The knowledge obtained in this study is intended to facilitate industry use of fluidized bed furnaces for the heat treatment of high carbon steel wire as well as aid future research on the topic. 


\section{CHAPTER 2}

\section{Theoretical Considerations}

\subsection{States of Fluidization}

Fluidization is a process that takes many stationary, solid particles and through the addition of a fluid with a certain velocity and mass flow that opposes the force of gravity, causes the mass of particles to rise and change to a fluid-like state. It is a gradual rather than a discrete process; as fluid flow is increased, it passes through several different states to go from a static packed bed to a fully fluidized state. When fluid flow is initialized, it begins to pass through the small gaps in between the solid particles. The particles offer resistance to the fluid flow through viscous friction, and as the flow increases, they rearrange themselves to offer less resistance and create paths in the otherwise packed bed for the fluid to flow through. Eventually, the velocity becomes high enough that the drag force on the particles is equal to the particle weight per bed area and the fluid has enough force to expand the bed. It is at this point that the system takes on fluid-like characteristics, and this state is known as incipient fluidization. Due to the packing and interlocking forces of particles, this does not occur exactly at the point where the pressure drop is first equal to the bed weight. The pressure drop continues to rise as fluid velocity is increased, up until the point where those forces are broken, after which it returns to being equal to the bed weight (Botterill, 1975). When visualized as a graph of pressure drop over fluid flow, this phenomenon can be seen as the hump like shape in Figure 2.1.

Further increases in fluid velocity lead to greater bed expansion, but no increase in pressure drop, as the particles are already fully suspended by the fluid. During this stage, different characteristics can be observed depending on the nature of the fluid. In the case of a liquid, expansion continues to be uniform. However, in the case of a gas, bubbles that contain fewer solid particles begin to form inside the fluidized bed. These bubbles are small and initially 


\subsection{States of Fluidization}

form at the distributor before rising to the surface. As they rise, the bubbles coalesce to form larger ones before finally bursting at the surface. Due to the fact that the bubbles contain no particles inside them, they force the density of the packing to increase locally around them. This aspect of the bubbles is what causes particle circulation in the gas fluidized bed, which leads to greater overall heat transfer rates (Botterill, 1975). The role that the bubbles play in the heat transfer varies and is largely dependent on the nature of the particles in the fluidized bed, and will be discussed in greater detail in the next section. At higher fluid velocities, the drag force on the particles begins to rise as particles become entrained in the fluid and carried out of the bed. This phenomenon is accompanied by a rise in pressure drop, and can be a desirable feature in some types of fluidized beds, such as circulating beds, in which departed particles are fed back into the bed. The various states of fluidization can be seen in Figure 2.1.

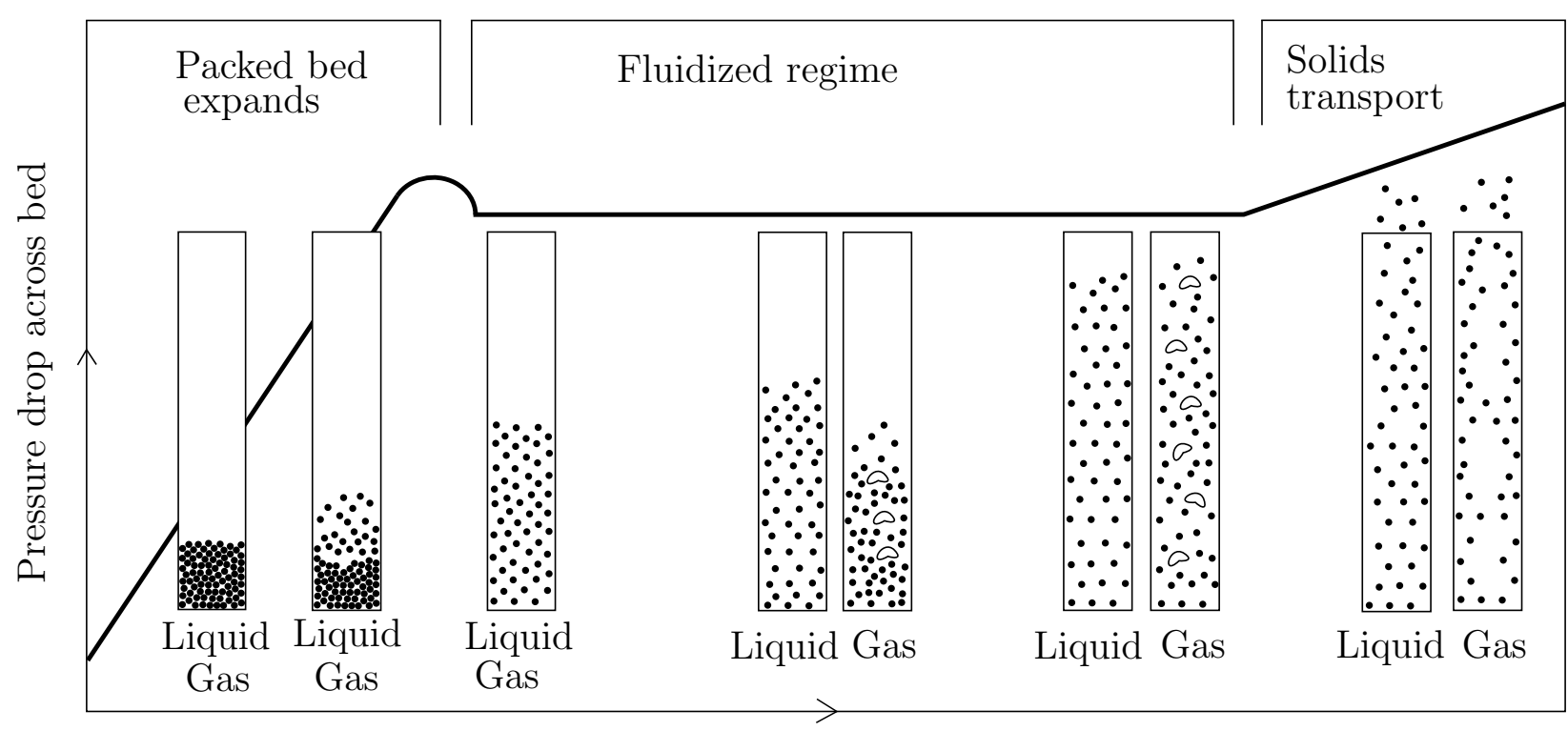

Fluid flow rate

Figure 2.1: Regimes of fluidization (Adapted from Botterill (1975)) 


\subsection{Classification of Particles}

\subsection{Classification of Particles}

The flow regime inside a fluidized bed is not only dependent on the fluid velocity, the particles play a crucial role as well. Geldart (1986) classified particles into four different groups, dependent on a combination of their density and size. The density is the difference between the solid and fluid densities, however it has been found that for gas fluidized beds at atmospheric pressure, fluid density is not high enough to influence the classification.

In order of increasing size, these groups are C, A, B and D, with group C representing fine powders, and group D large particles. Each group of particles occupies a region on a graph of density vs diameter as can be seen in Figure 2.2. In addition to their physical characteristics, each group displays different fluidization characteristics. Particles belonging to Group C are very fine and difficult to fluidize, due to their highly cohesive nature. This cohesiveness leads to plug like motion in small diameter beds, or channeling in larger diameter ones. In this case the inter-particle forces exceed those applied by the fluid, and the packing cannot be easily broken. Additional aides are often needed to fluidize these types of particles, such as mechanical stirrers, or the addition of other particles. (Geldart, 1986)

Group A has been studied extensively in the past, due to their commercial usage in fluidized bed catalytic reactors. These powders exhibit significant inter-particle forces, but unlike Group C, fluidize without too much difficulty. Due to these inter-particle forces, Group A powders exhibit a large degree of expansion between the point where fluidization occurs, to the point where bubbling commences. The bubbles in a bed filled with Group A powders have a higher velocity than the fluidizing gas and produce large quantities of solids mixing. (Geldart, 1986)

Group B particles, such as sand, have good fluidization characteristics, and particle circulation. The particle interlocking forces are negligible in this group, and bubbling occurs almost immediately after fluidization. Due to the low inter-particle forces, fluidized beds of Group B particles do not experience much expansion, and collapse quickly when the fluid is stopped. Bubbles in beds of Group B particles have a higher velocity than the fluidizing gas, and their size is proportional to both gas velocity and bed height.

Group D consists of the largest and most dense particles, which are difficult to fluidize due 


\subsection{Classification of Particles}

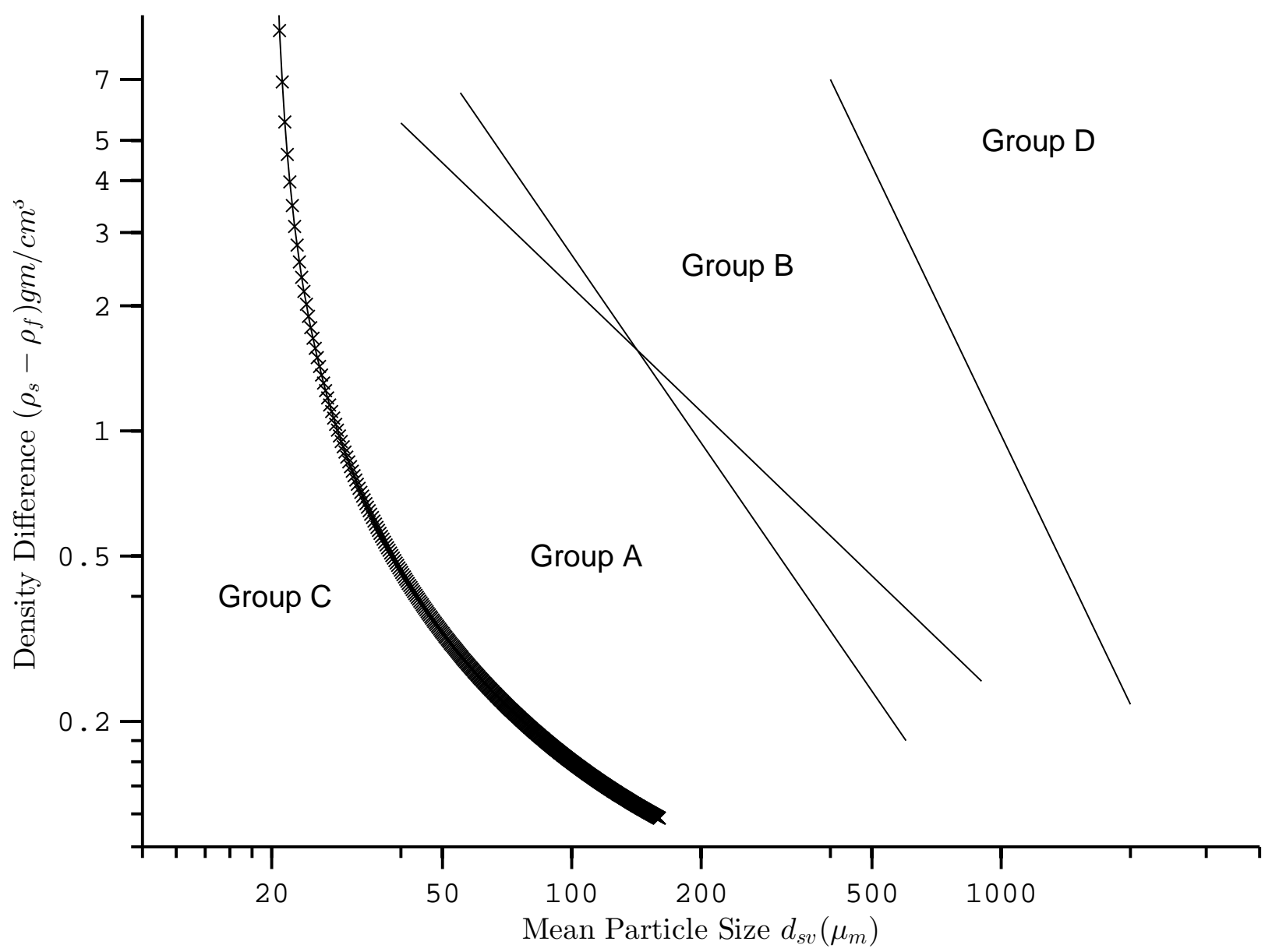

Figure 2.2: Geldart's classification of powders (Adapted from Gupta and Sathiyamoorthy (1998))

to their size and weight. For the most part, bubbles have a lower velocity than the fluidizing gas, causing the gas to flow through the bubbles as well, which does not occur in the other groups. This causes lower particle circulation, which in turn means that the heat transfer mechanisms in beds filled with Group D particles are different than for A and B. 


\subsection{Pressure Drop Through a Bed}

As discussed previously, at the point of incipient fluidization, the drag force on the particles is equal to the weight of the bed, as the fluid exhibits just enough force to suspend the particles (Botterill, 1975). This relationship between the fluid and particles can be expressed as:

$$
A_{b} \Delta p=\frac{m}{\rho_{s}}\left(\rho_{s}-\rho_{g}\right)
$$

with: $A_{b}=$ Effective Bed Area $\left(m^{2}\right)$

$$
\begin{aligned}
\Delta p & =\text { Pressure Drop Across Bed }(P a) \\
m & =\text { Mass of Particles in Bed }(k g) \\
\rho_{s} & =\text { Density of Solid Particles }\left(\frac{k g}{m^{3}}\right) \\
\rho_{g} & =\text { Density of Gas }\left(\frac{k g}{m^{3}}\right) \\
g & =\text { Acceleration due to Gravity }\left(9.81 \frac{m}{s^{2}}\right)
\end{aligned}
$$

In the case of a gas fluidized bed at atmospheric pressure, the gas density is very low compared to the particle density, so Equation 2.1 can be reduced to:

$$
A_{b} \Delta p=m g
$$

The pressure drop across the bed can be expressed as a combination of bed height and voidage, which in turn gives

$$
\Delta p=(1-\varepsilon) g \rho_{s} H
$$


where $: \varepsilon \equiv$ (Volume of Bed - Volume of Particles)/Volume of Bed

$$
\begin{aligned}
\varepsilon & =1-\frac{\rho_{b}}{\rho_{s}} \\
\rho_{b} & =\text { Bulk Density of Bed }\left(\frac{k g}{m^{3}}\right) \\
H & =\text { Height of Bed }(m)
\end{aligned}
$$

The bulk density that appears in the expression for voidage is an overall density that includes both the solid particles and the voids within the bed, not to be confused with the particle density, which is simply based on the volume and shape of the particle.

A noteworthy fact about Equation 2.3 is that it implies that the pressure gradient in the bed is not dependent on the fluid velocity, which contrasts the findings of workers such as Carman, Kozeny and Ergun.

Ergun developed an equation, which was proven to be valid for different flow conditions, and it shows the proportionality between the fluid velocity and pressure gradient in the expanding regime of a packed bed (Geldart, 1986). This equation is known as the Ergun equation, and is commonly used to represent the flow conditions inside a fluidized bed.

$$
\frac{\Delta p}{H}=\frac{p_{i n}}{\bar{p}}\left[150 \frac{(1-\varepsilon)^{2}}{\varepsilon^{3}} \frac{\mu_{g} U}{d_{s v}^{2}}+1.75 \frac{1-\varepsilon}{\varepsilon^{3}} \frac{\rho_{g} U^{2}}{d_{s v}}\right]
$$

where $: \frac{p_{i n}}{\bar{p}}=$ Correction Factor for Compressibility

$p_{i n}=$ Absolute Pressure at Bed Inlet $(\mathrm{Pa})$

$\bar{p}=$ Average Absolute Pressure in the $\operatorname{Bed}(P a)$

$\mu_{g}=$ Gas Viscosity $\left(\frac{k g}{m s}\right)$

$U=$ Fluidizing Velocity $\left(\frac{m}{s}\right)$

$d_{s v}=$ Diameter of a Sphere with the same Surface/Volume Ratio as the particle $\left(m^{2}\right)$

$d_{p}=$ Mean Particle Diameter $\left(m^{2}\right)$ 


\subsection{Minimum Fluidization Velocity}

The Ergun equation consists of two main terms, the first representing laminar flow conditions and the second turbulent. As the flow regime moves toward the laminar or turbulent end, the respective term grows to dominate the equation. In the case of laminar flow $\left(\operatorname{Re}_{p}<1\right.$,

$\left.\operatorname{Re}_{p}=\frac{\rho_{g} d_{p} U}{\mu_{g}}\right)$, when the turbulent term is negligible, the Ergun equation becomes the Carman-Kozeny equation (Geldart, 1986).

$$
\frac{\Delta p}{H}=\frac{K \mu_{g}}{d_{p}^{2}} \frac{(1-\varepsilon)^{2}}{\varepsilon^{3}} U
$$

where: $K=180$ for $0.4 \leq \varepsilon \leq 0.5$ and $0.1 \leq \operatorname{Re}_{p} \leq 1.0$

The Ergun equation is a useful tool that can be used to determine the pressure drop at any vertical location inside a fluidized bed. Alternatively, experimental methods can be utilized to accomplish the same goal.

\subsection{Minimum Fluidization Velocity}

As discussed in Section 2.3, the pressure drop across a fluidized bed increases linearly with increasing fluid velocity, up until the point of incipient fluidization. The flow inside the bed would be laminar over this range $\left(\operatorname{Re}_{p}<1\right)$, and indeed, based on the Carman-Kozeny equation (2.5) this linear relationship between the pressure drop and fluid velocity can be observed. At the point of incipient fluidization, this no longer holds true, and the velocity at this point is defined as the Minimum Fluidization Velocity $\left(U_{m f}\right) . U_{m f}$ can be predicted by substituting the expression relating pressure to bed height and voidage, Equation 2.3, into the Ergun equation and setting the pressure at the bed inlet equal to the mean pressure in the bed. The result is:

$$
\frac{\rho_{g} d_{p}^{3}\left(\rho_{s}-\rho_{g}\right) g}{\mu_{g}^{2}}=\frac{150\left(1-\varepsilon_{m f}\right)}{\varepsilon_{m f}^{3}} \frac{\rho_{g} d_{p}}{\mu_{g}} U_{m f}+\frac{1.75}{\varepsilon_{m f}^{3}} \frac{\rho_{g}^{2} d_{p}^{2}}{\mu_{g}^{2}} U_{m f}^{2}
$$




$$
\text { where : } \begin{aligned}
U_{m f} & =\text { Minimum Fluidization Velocity }\left(\frac{m}{s}\right) \\
\varepsilon_{m f} & =\text { Bed Voidage at } U_{m f} \\
\operatorname{Ar} & =\frac{\rho_{g} d_{p}^{3}\left(\rho_{s}-\rho_{g}\right) g}{\mu_{g}^{2}} \\
\operatorname{Re}_{m f} & =\frac{\rho_{g} d_{p}}{\mu_{g}} U_{m f}
\end{aligned}
$$

Making the substitutions for $\mathrm{Ar}$ and $\mathrm{Re}_{m f}$ in Equation 2.6 leads to:

$$
\operatorname{Ar}=\frac{150\left(1-\varepsilon_{m f}\right)}{\varepsilon_{m f}^{3}} \operatorname{Re}_{m f}+\frac{1.75}{\varepsilon_{m f}^{3}} \operatorname{Re}_{m f}^{2}
$$

where the left hand side is the dimensionless term known as the Archimedes number and the two terms on the right hand side before $\operatorname{Re}_{m f}$ and $\operatorname{Re}_{m f}^{2}$ are constants that are determined empirically. There exist slight discrepancies in the values of these constants between different workers, however a commonly accepted set of constants was found by Wen and Yu (Geldart, 1986) leading to:

$$
\mathrm{Ar}=1650 \mathrm{Re}_{m f}+24.5 \mathrm{Re}_{m f}^{2}
$$

which can be rearranged into Equation 2.9 to isolate $U_{m f}$ :

$$
U_{m f}=\frac{\mu_{g}}{\rho_{g} d_{p}}\left[(1135.7+0.0408 \mathrm{Ar})^{\frac{1}{2}}-33.7\right]
$$

The range of applicability of Equation 2.9 is limited to particles of diameter greater than $100 \mu m$, such as solids from Geldart groups B and D.

\subsubsection{Effect of Pressure and Temperature on $U_{m f}$}

By observing Equation 2.9, it can be seen that while pressure and temperature do not appear directly, the gas density and gas viscosity appear, and as such pressure and temperature can influence $U_{m f}$ indirectly. Kunii and Levenspiel (1977) and Geldart (1986) studied the effects of pressure on minimum fluidization velocity, and found that it had a negligible impact, except for the case of large particles belonging to Group D, in which case an increase in 


\subsection{Minimum Fluidization Velocity}

pressure led to a decrease in minimum fluidizing velocity. Temperature was found to play a much more significant role by Botterill (1975) and Saxena (1989), who found that an increase in temperature is accompanied by a decrease in minimum fluidizing velocity. Their findings can be verified using Equation 2.9. Figure 2.3 is a contour plot of $U_{m f}$ vs temperature and pressure, for a Geldart Group B particle of $250 \mu \mathrm{m}$ diameter and a density of $3970 \mathrm{~kg} / \mathrm{m}^{3}$. It can be seen that the contour lines are nearly parallel to the pressure axis, showing that there is a strong dependence on temperature, but only a slight one on pressure.

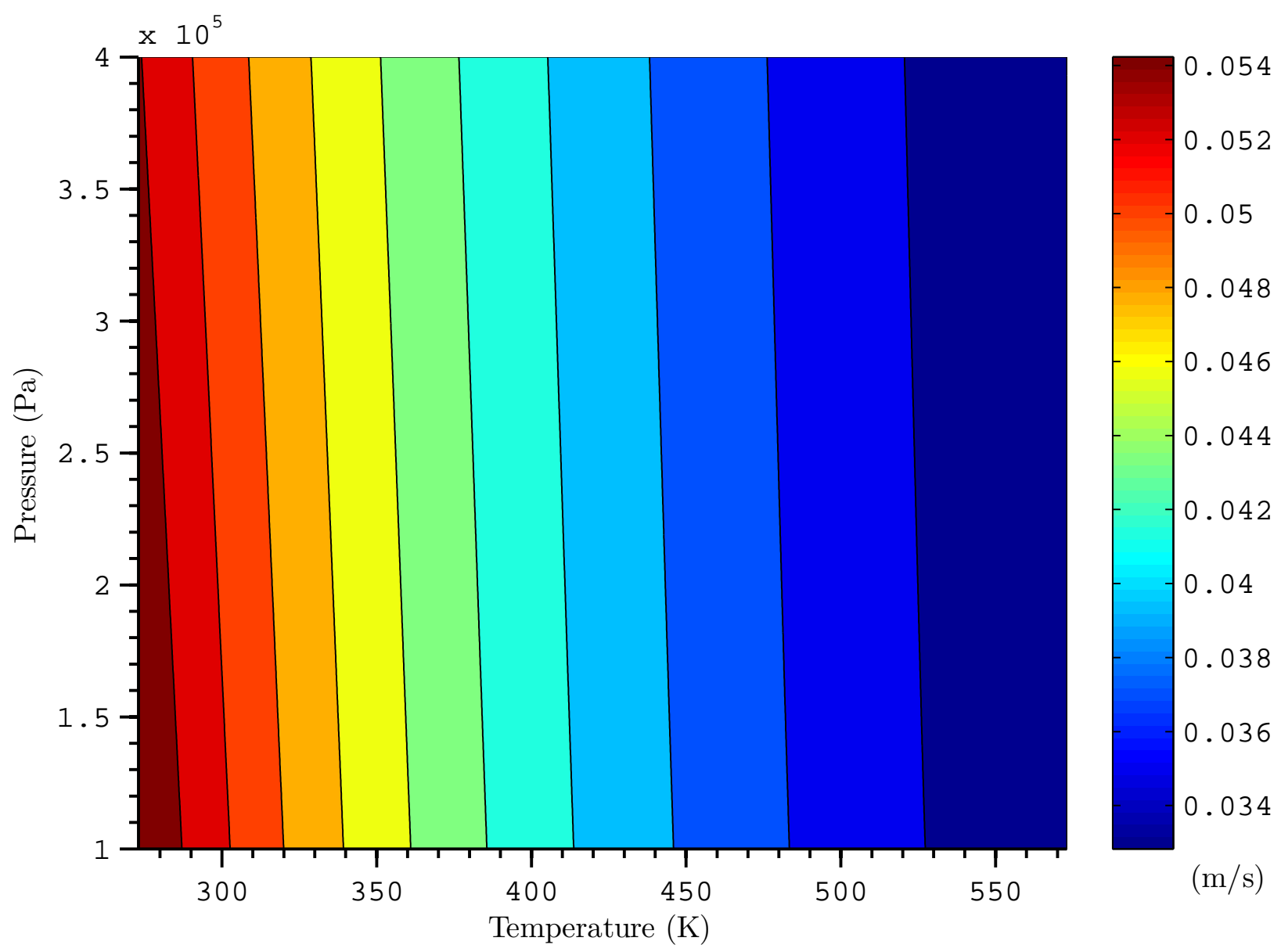

Figure 2.3: Minimum fluidization velocity at different temperatures and pressures for a bed of Geldart Group B particles fluidized in air 


\subsection{Heat Transfer in Fluidized Beds}

The particles in a fluidized bed offer a very large exposed solid surface area to the fluid flow, allowing rapid heat transfer between the solid particles and the fluid. Fluidized beds also boast high heat capacity (on the order of $10^{6} \mathrm{~J} / \mathrm{m}^{3} \mathrm{~K}$ ), with heat transfer coefficients in the range of 250-700 W/ $\mathrm{m}^{2} K$ (Gupta and Sathiyamoorthy, 1998). As discussed earlier, the bubbling regime that forms in gas fluidized beds causes a high degree of solids mixing, which leads to low temperature gradients, meaning very uniform temperatures throughout the fluidized bed. Due to these factors, it is possible to obtain high rates of heat transfer to surfaces immersed in fluidized beds (Geldart, 1986).

\subsubsection{Heat Transfer Mechanisms}

There are three modes of heat transfer to surfaces immersed in a fluidized bed: particle convection, gas convection and radiation. Particle convection refers to the unsteady-state conduction through the small volume of gas between the particle and surface; often called a gas lens (Zabrodsky, 1966), and not the direct particle to surface conduction. The latter is negligible due to the very small contact area (Saxena, 1989). Gas convection and radiation are the forced convection from the gas passing over the surface and radiative heat transfer to the surface from the particles, respectively. The magnitude of all of these modes of heat transfer depend on the flow conditions, properties of the particles and temperature (Gupta and Sathiyamoorthy, 1998). All of the aforementioned modes of heat transfer can be considered independent and their effects can be added to give a total heat transfer coefficient. They are pictured in Figure 2.4. There are two extreme cases when it comes to modes of heat transfer. For Group A, which consists of fine particles, particle convection dominates. As previously mentioned, the bubble velocity in a bed of Group A particles is much higher than the gas velocity, which causes a very high degree of particle circulation, resulting in very high particle convection. The particles also have much greater heat capacity than the gas, and as such are responsible for the bulk of the heat transfer throughout the bed (Geldart, 


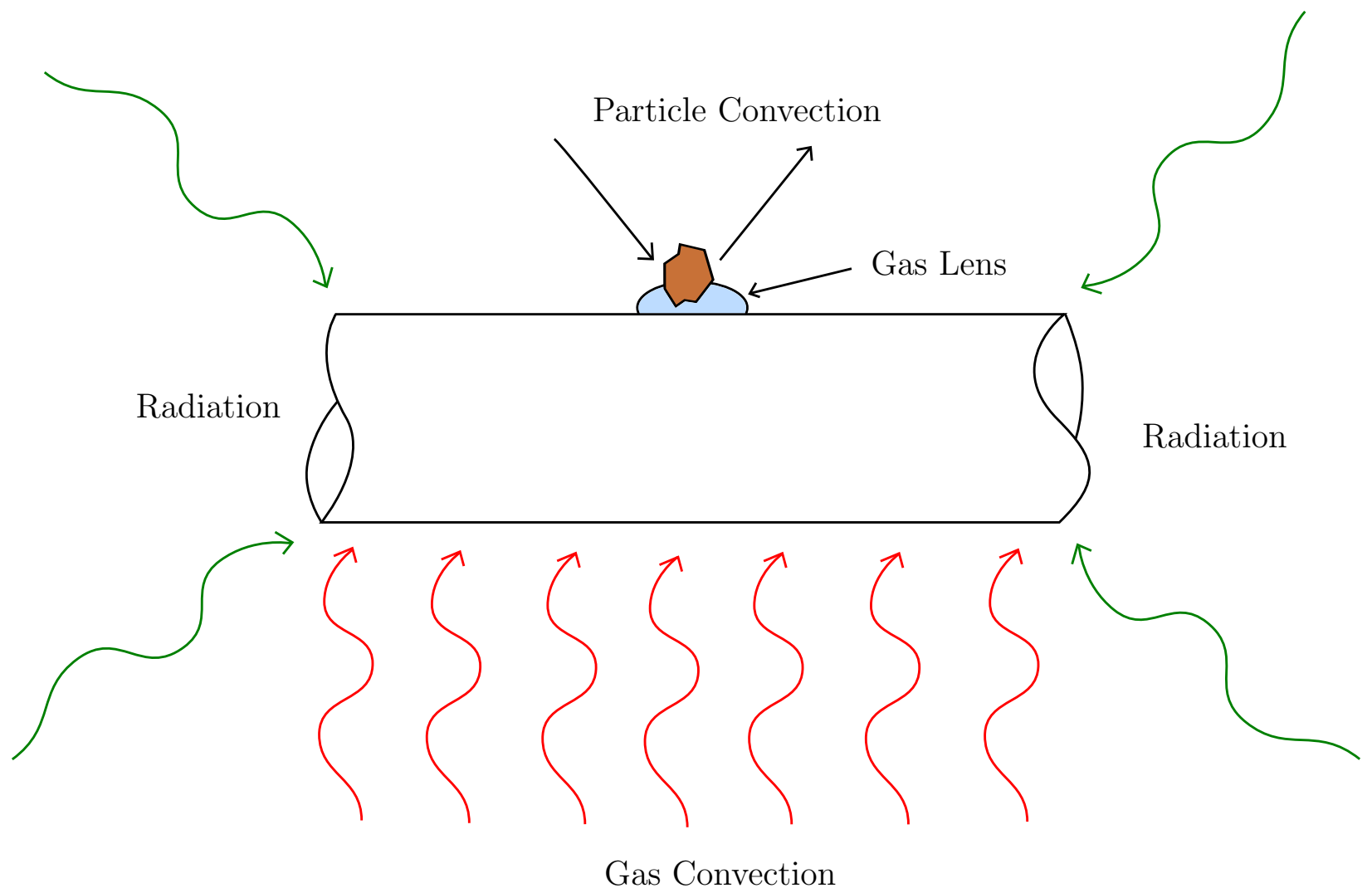

Figure 2.4: Modes of heat transfer in gas fluidized beds

1986). The gas flows through the interstices of the particles and allows for heat transfer between the particles themselves (Gupta and Sathiyamoorthy, 1998) as well as maintaining the particle circulation.

In the case of Group D, which comprises large, heavy particles which have a high minimum fluidizing velocity, the gas velocity is higher than the bubble velocity, leading to poor particle circulation and gas convection is the primary mechanism of heat transfer (Martin, 1984). Further velocity increases cause the flow to become turbulent (Saxena, 1989), which is typical of pressurized fluidized beds. For an immersed cylinder, this promotes a blowing effect, lowering the heat transfer coefficient (Molerus and Wirth, 1997).

Particles belonging to groups $\mathrm{B}$ and $\mathrm{C}$ are subject to a mix of particle and gas convection. Increasing the gas velocity causes an increase in gas convection, but it also has an influence 


\subsection{Heat Transfer in Fluidized Beds}

on particle convection. When gas velocity is increased, it causes the particle residence time at the surface, $\tau$, to decrease, and the bed voidage, $\varepsilon$, to increase. Shorter particle residence times allow fresh particles to come into contact with the surface more often, boosting heat transfer rates. There is however an optimal time, based on the volumetric heat capacity of the particles and the thermal conductivity of the gas that ensures the maximum amount of heat transfer. Shorter residence times than this would not allow the particles to transfer all of their heat energy onto the surface, resulting in a lower heat transfer rate. The effect that this has on particle convection, is that it increases with gas velocity, up to a maximum value, $h_{w \max }$, followed by a decrease with any further increase in gas velocity (Grewal and Saxena, 1980).

\section{Effects of Wire Movement}

In the case of a static surface, the gas velocity would be the only parameter affecting the residence time. However if the surface were to be moving at a velocity of a similar magnitude as the gas velocity, it could alter the particle residence time and play a significant role in the heat transfer. Figure 2.5 shows a visual representation of this, with a highlighted region representing the duration $\tau$ that the particle makes with the surface. The size of the region represents the time, while the shape is meant to illustrate the effects that the wire movement speed and fluidization velocity would have on the duration respectively. The bottom left quadrant represents the longest duration, when the fluidizing velocity is low and the wire is static. The top right is the opposite, with high fluidizing velocity as well as wire movement. The top left and bottom right represent the intermediate states where either wire speed or fluidizing velocity are acting to reduce the duration of $\tau$.

Due to the different relationships that particle and gas convection have with gas velocity, the peak heat transfer occurs at some intermediate point where neither value is necessarily at its maximum, but their sum is. Finally, despite its importance, gas velocity is not the only factor to consider, particle diameter also plays a role, having an inverse relationship with the heat transfer coefficient. 


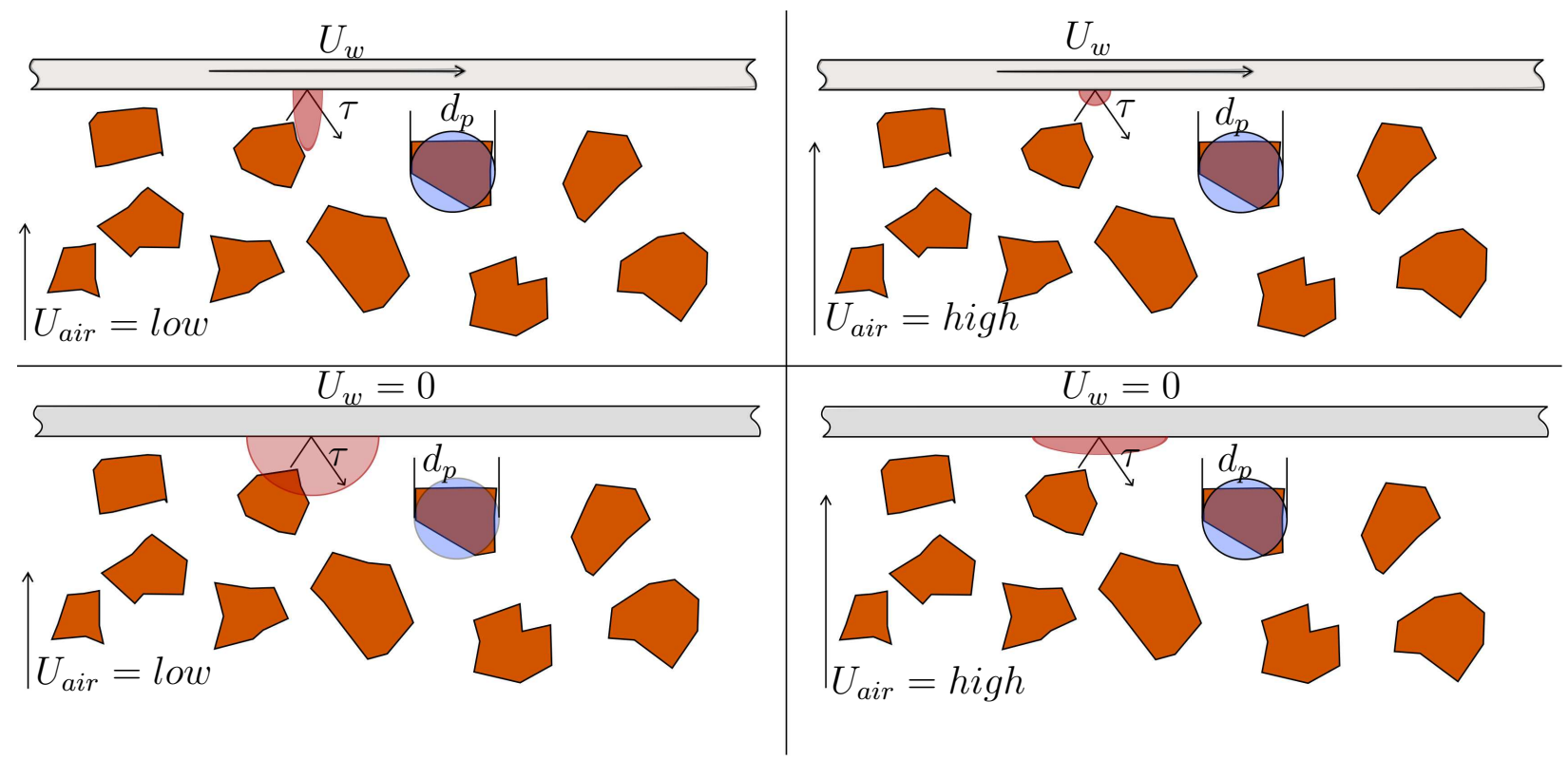

Figure 2.5: Effects of air velocity and wire speed on particle convection

Radiation can also play an important role in heat transfer, in the case of high temperature operation (above $675 \mathrm{~K}$ ). Radiative heat transfer has a non-linear dependence on temperature, and as such is difficult to include directly in a correlation. However, since radiative heat transfer to an immersed surface depends on the particles near the surface and bubbling gas fluidized beds have excellent temperature uniformity, it is possible to consider the radiative component separately and add it to the two convective components (Saxena, 1989).

\subsubsection{Heat Transfer Correlation for Immersed Surfaces}

The most commonly used correlation, due to its ability to predict a very large range of data is the Grewal and Saxena (1980) correlation. While this correlation is meant for medium to large sized cylinders, the authors claim it is able to predict heat transfer to small cylinders within $\pm 25 \%$ as well, if immersed in Geldart Group A or B particles. The correlation is 
given below:

$$
\begin{aligned}
\mathrm{Nu}=47(1-\varepsilon)\left[\left(\frac{G D_{t} \rho_{s}}{\rho_{g} \mu_{g}}\right)\left(\frac{\mu_{g}^{2}}{d_{p}^{3} \rho_{s}^{2} g}\right)\right]^{0.325}\left[\frac{\rho_{s} C_{p, s} D_{t}^{\frac{3}{2}} g^{\frac{1}{2}}}{k_{g}}\right]^{0.23} \operatorname{Pr}^{0.3} \\
\text { where : } \begin{aligned}
N u & =\text { Nusselt Number } \equiv \frac{h D_{t}}{k_{g}} \\
h & =\text { Heat Transfer Coefficient }\left(\frac{W}{m^{2} K}\right) \\
D_{t} & =\text { Diameter of Immersed Surface }(m) \\
k_{g} & =\text { Thermal Conductivity of the Fluidizing Gas }\left(\frac{W}{m K}\right) \\
G & =\text { Mass Flow Rate of the Fluidizing Gas }\left(\frac{k g}{s}\right) \\
C_{p, s} & =\text { Specific Heat Capacity of Particles }\left(\frac{J}{k g K}\right) \\
\operatorname{Pr} & =\text { Prandtl Number of the Fluidizing Gas } \equiv \frac{C_{p, g} \mu_{g}}{k_{g}}
\end{aligned}
\end{aligned}
$$

The Grewal and Saxena correlation differs from previous correlations because it includes two components that are not typically present in others; the volumetric heat capacity of the particles, and the bed voidage, $\varepsilon$. The volumetric heat capacity of the particles has a direct influence on what the ideal particle residence time, $\tau$, would be in order to maximize heat transfer, and as previously discussed, both $\tau$ and $\varepsilon$ affect the particle convection heat transfer. Voidage is defined by Saxena (1989) as Equation 2.11.

$$
\varepsilon=\frac{1}{2.1}\left[0.4+\left\{4\left[\frac{\mu_{g} G}{d_{p}^{2}\left(\rho_{g}\left(\rho_{s}-\rho_{g}\right)\right) \phi_{s}^{2} g}\right]^{0.43}\right\}^{1 / 3}\right]
$$


where $: \phi_{s}=$ Sphericity of the Particles

$$
\phi_{s} \equiv \frac{\text { Surface Area of a Sphere of Equivalent Volume }}{\text { Surface Area of the Particle }}
$$

As can be seen from Equation 2.11, the mass flow rate is a key parameter in determining voidage, as well as the absolute viscosity of the gas. In addition, it includes the particle sphericity, $\phi$, which is a function of the particle shape. Due to the relationship between particle sphericity and voidage, when the bed is at minimum fluidizing velocity, one can be used to find the other based on Figure 2.6 developed by Kunii and Levenspiel (1977).

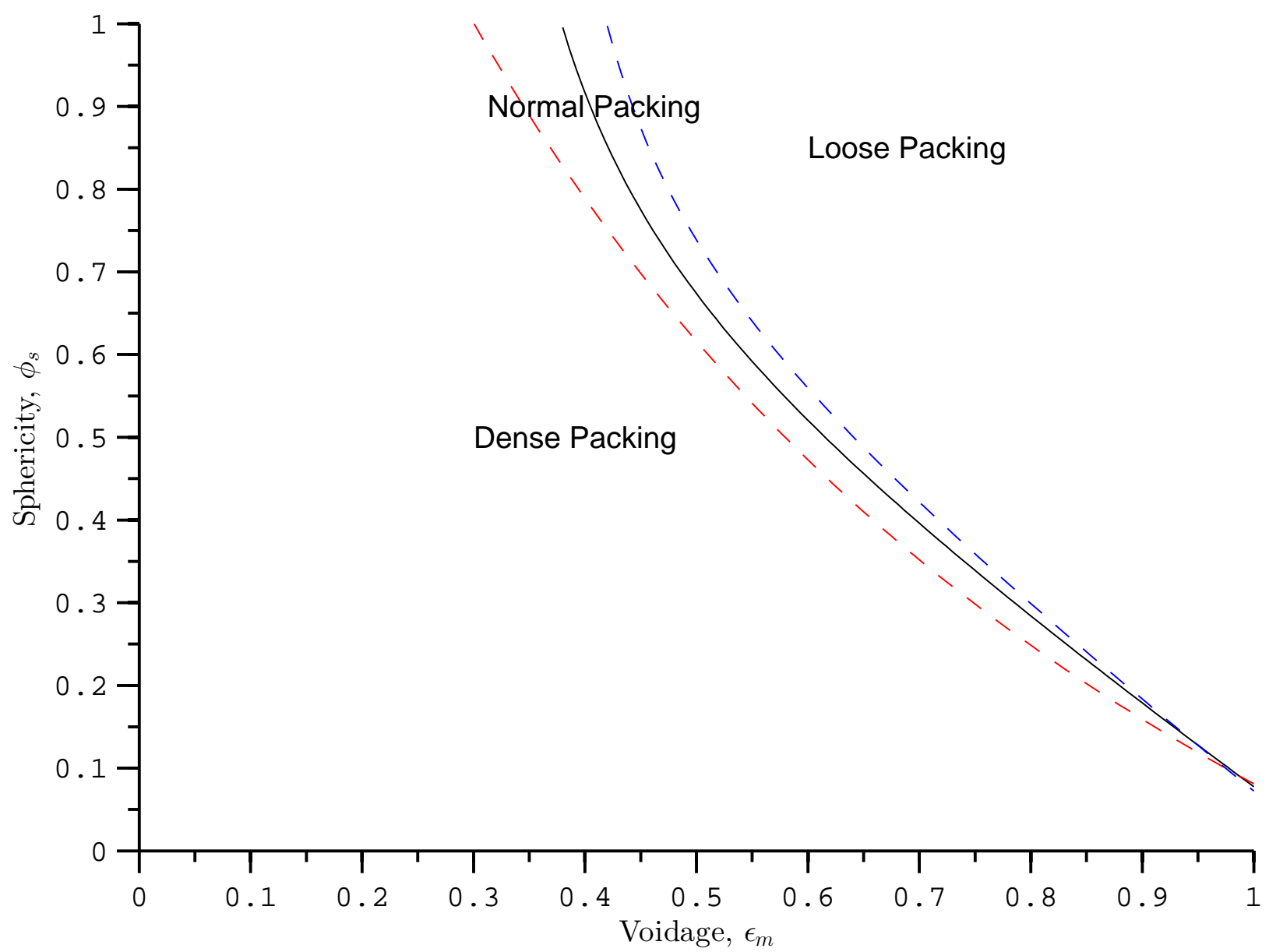

Figure 2.6: Sphericity vs bed voidage (Adapted from Kunii and Levenspiel (1977)) 


\section{Small Cylinders}

Despite the successes of the Grewal and Saxena correlation for predicting heat transfer to large and medium sized tubes, and claims of applicability to small cylinders, a study by Friedman et al. (2006) showed that all correlations based on large diameter tubes, including that of Grewal and Saxena grossly over-predict heat transfer rates when applied to small diameter cylinders. Figure 2.7 contains the experimental data obtained by Friedman et al. (2006), as well as plots of many popular correlations demonstrates their inadequacy when applied to small diameter wire. Figure 2.7 also shows that in addition to over-predicting the Nusselt number, the shape of the plots of all of the correlations does not match that of the experimental data. All of the correlations show a steady increase in Nusselt number with increasing fluid velocity, while the experimental data shows a plateau and eventually a decrease.

As previously mentioned, Friedman et al. (2006) concluded that for small cylinders, fluidizing velocity and bed voidage do not play as large a role as previously thought, and the key parameters are the Archimedes number and the ratio of cylinder diameter to particle diameter.

The correlation put forward was as follows:

$$
\mathrm{Nu}_{\text {mean }}=C A r^{n} \frac{D_{t}}{d_{p}}
$$

with $C=1.35$ and $n=0.15$. This correlation is a very simple one, intended for engineering applications, however when compared to the Grewal and Saxena correlation (Equation 2.10), it is capable of predicting the Nusselt number much better for smaller cylinders, and even predicts Grewal and Saxena's own data for a $12.7 \mathrm{~mm}$ cylinder quite well (Friedman et al., 2006). At large values of $A r^{n} \frac{D_{t}}{d_{p}}(>100)$ however, which is typical for large diameter cylinders, Friedman's correlation begins to deviate from the data, and the Grewal and Saxena correlation is a better fit.

A newer correlation by Masoumifard et al. (2008) based on cluster renewal and penetration depth also exists that can effectively predict data over a wide range of cylinder diameters. 


\subsection{Heat Transfer in Fluidized Beds}

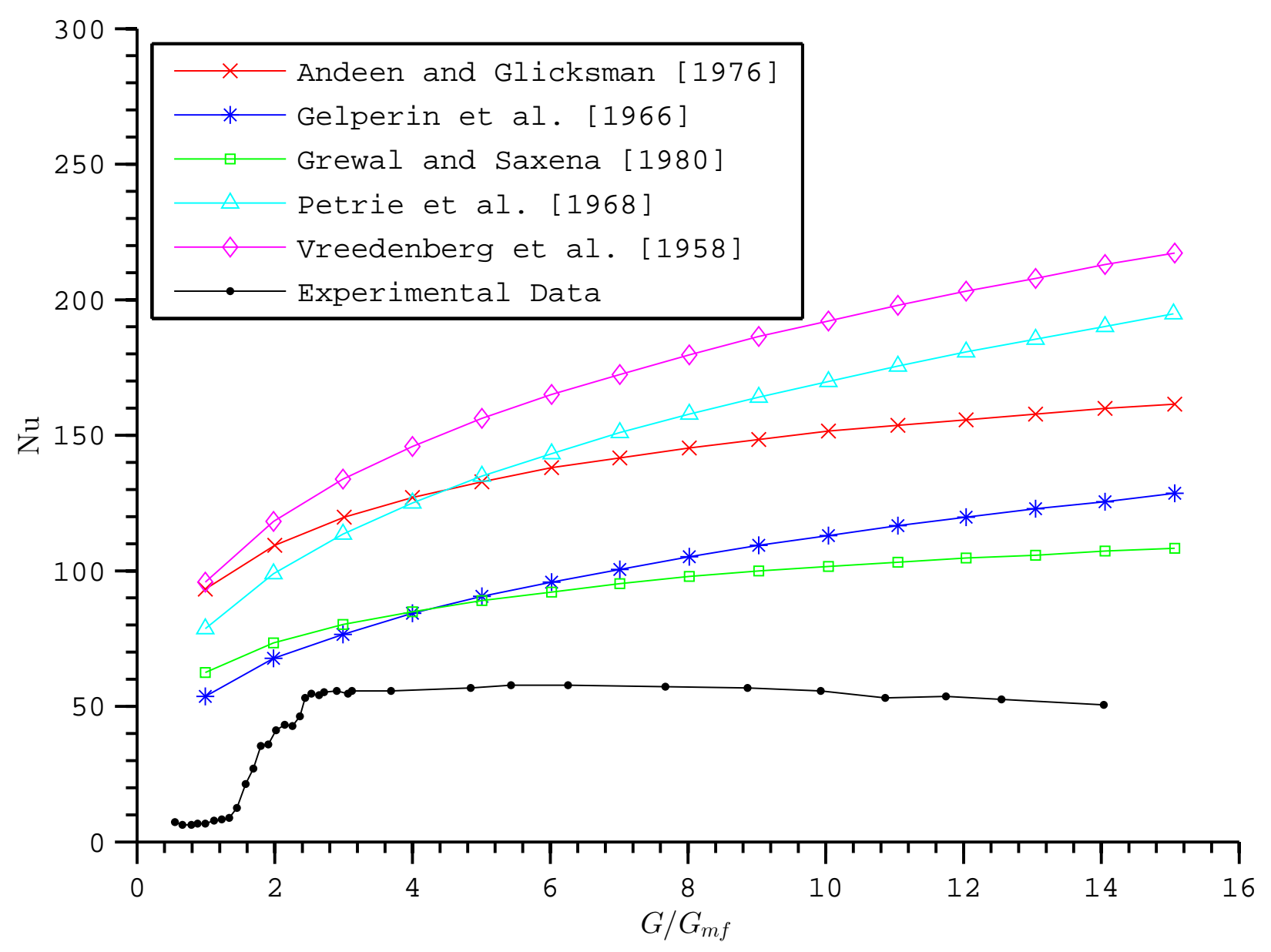

Figure 2.7: A comparison of Friedman's experimental data with existing correlations (Adapted from Friedman et al. (2006))

Due to the nature of the cluster renewal method, this correlation is quite a bit more involved but it illustrates rather well the role that the contact time between the particles and the surface plays in the heat transfer. The correlation was developed for low temperature fluidized beds where radiation does not play a role $\left(<600^{\circ} \mathrm{C}\right)$ and begins by splitting the remaining modes of heat transfer; particle and gas convection:

$$
\bar{h}_{c}=(1-f) \bar{h}_{c c}+(f) h_{g c}
$$




$$
\begin{aligned}
& \text { where }: \bar{h}_{c}=\text { Overall Heat Transfer Coefficient }\left(\frac{W}{m^{2} K}\right) \\
& \bar{h}_{c c}=\text { Heat Transfer Coefficient due to Particle Convection }\left(\frac{W}{m^{2} K}\right) \\
& h_{g c}=\text { Heat Transfer Coefficient due to Gas Convection }\left(\frac{W}{m^{2} K}\right) \\
& f=\text { Bubble Fraction }=0.19\left[\frac{d_{p} g}{U_{m f}^{2}\left(\frac{U_{0}}{U_{m f}}\right)^{2}}\right]^{-0.23} \\
& U_{0}=\text { Fluidizing Velocity }\left(\frac{m}{s}\right)
\end{aligned}
$$

The gas convective component, $h_{g c}$ is based on a correlation by Baskakov et al. (1973):

$$
h_{g c}=0.009\left(\frac{k_{g}}{d_{p}}\right) \operatorname{Ar}^{0.5} \operatorname{Pr}^{0.33}
$$

The particle convection component then occurs over two distinct time periods, based on whether the heat flux has completely diffused inside the cluster from the surface to the bulk of the bed. The time it takes for the heat flux to be completely diffused inside the cluster is termed $t_{0}$, and the periods are divided into $t<t_{0}$ and $t>t_{0} . t_{0}$ is defined as:

$$
t_{0}=\frac{d_{c}^{2}}{6 \alpha_{c}}
$$




$$
\text { where: } \begin{aligned}
\alpha & =\text { Thermal Diffusivity of the Cluster }\left(\frac{m^{2}}{s}\right) \\
& =\left(\frac{k_{c}}{\rho_{c}} C_{C}\right) \\
d_{c} & =\text { Average of Diameters of Descending and Ascending Clusters }
\end{aligned}
$$

Descending dia $(m)=\frac{e^{-1.79+1.555\left(U_{0}-U_{m f}\right)}}{1000}$

Ascending dia $(m)=\frac{e^{-0.47+1.276\left(U_{0}-U_{m f}\right)}}{1000}$

The first period is referred to as the partial penetration and represented by:

$$
\begin{aligned}
& \bar{h}_{c c_{t<t_{0}}}=\frac{2}{3 \tau}\left(k_{c} \rho_{c} C_{c}\right)\left(\frac{\delta}{k_{g}}\right) \times \ln \left[\frac{4\left(\frac{\delta}{k_{g}}\right)^{2}\left(k_{c} \rho_{c} C_{c}\right)}{\left(4\left(\frac{\delta}{k_{g}}\right)^{2}\left(k_{c} \rho_{c} C_{c}\right)-6 \tau\right)} \frac{1-\frac{\sqrt{6}}{2\left(\frac{\delta}{k_{g}}\right)}\left(\frac{\tau}{k_{c} \rho_{c} C_{c}}\right)^{0.5}}{1+\frac{\sqrt{6}}{2\left(\frac{\delta}{k_{g}}\right)}\left(\frac{\tau}{k_{c} \rho_{c} C_{c}}\right)^{0.5}}\right] \\
& +\frac{2 \sqrt{6}}{3 \tau}\left(\left(k_{c} \rho_{c} C_{c}\right) \tau\right)^{0.5} \\
& \text { where : } \tau=\text { Cluster-wall contact time }(s)=1.2\left[\frac{d_{p} g}{U_{m f}^{2}\left(\frac{U_{0}}{U_{m f}}\right)^{2}}\right]^{0.3}\left(\frac{d_{p}}{D_{t}}\right)^{0.225} \\
& k_{c}=\text { Thermal Conductivity of the Cluster }\left(\frac{W}{m K}\right) \\
& =k_{g}\left(1-\sqrt{1-\varepsilon_{c}}+\sqrt{1-\varepsilon_{c}}\left(\frac{k^{\prime}}{k_{g}}\right)\right)
\end{aligned}
$$




$$
\begin{aligned}
\rho_{c} & =\text { Density of the Cluster }\left(\frac{k g}{m^{3}}\right) \\
& =\left(1-\varepsilon_{c}\right) \rho_{s}+\varepsilon_{c} \rho_{g} \\
C_{c} & =\text { Specific Heat Capacity of the Cluster }\left(\frac{J}{k g K}\right) \\
& =\left(1-\varepsilon_{c}\right) C_{p, s}+\varepsilon_{c} C_{p, g} \\
C_{p, g} & =\text { Specific Heat Capacity of the Gas }\left(\frac{J}{k g K}\right) \\
\varepsilon_{c} & =\text { Voidage of the Cluster } \\
& =1-\frac{\left(1-\varepsilon_{m f}\right)\left[0.7293+0.5139\left(\frac{d_{p}}{D_{t}}\right)\right]}{1+\left(\frac{d_{p}}{D_{t}}\right)} \\
\frac{k^{\prime}}{k_{g}} & =\frac{2}{1-B \frac{k_{g}}{k_{s}}}\left[\frac{\left(1-\frac{k_{g}}{k_{s}}\right) B}{\left(1-B \frac{k_{g}}{k_{s}}\right)^{2}} \ln \left(\frac{k_{s}}{B k_{g}}\right)-\left(\frac{B+1}{2}\right)-\frac{B-1}{\left(1-B \frac{k_{g}}{k_{s}}\right)}\right] \\
B & =1.25\left(\frac{1-\varepsilon_{c}}{\varepsilon_{c}}\right)\left(\frac{10}{9}\right) \\
\delta & =\text { Gas Gap Thickness }(m) \\
& =\frac{d_{p}}{10}\left(1+\frac{1}{2}|\cos \theta|\right)
\end{aligned}
$$

In the case that the total cluster-wall contact time $\tau$ is less than $t_{0}$, Equation 2.16 can be used to calculate the total particle convection contribution to heat transfer. If $\tau$ is greater than $t_{0}$, the second period needs to be considered as well. It occurs after the heat flux has fully diffused inside the cluster, and is referred to as the total heat penetration period.

$$
\begin{aligned}
\bar{h}_{c c_{t}>t_{0}}= & \frac{2}{\left[C_{3}+\frac{2 d_{c}}{k_{c}} C_{1}\right]}\left\{1-\frac{1}{C_{2}\left(\tau-t_{0}\right)}\right. \\
& \left.\times \ln \left[1+\frac{\frac{2 d_{c}}{k_{c}} C_{1}}{C_{3}}\left(1-\exp \left(C_{2}\left(\tau-t_{0}\right)\right)\right)\right]\right\}
\end{aligned}
$$




$$
\text { where : } \begin{aligned}
C_{1} & =\frac{1}{2}+\frac{1}{\mathrm{Bi}} \\
C_{2} & =-\frac{m}{1+\frac{\mathrm{Bi}}{3}} \\
C_{3} & =\frac{2 \delta}{k_{g}}+\frac{d_{c}}{k_{c}} \\
m & =\frac{h_{g c}}{\rho_{c} C_{c} d_{c}} \\
\mathrm{Bi} & =\text { Biot Number }=\frac{h_{g} d_{c}}{k_{c}}
\end{aligned}
$$

To determine the total particle convection heat transfer, the two periods are scaled based on their respective duration and added together:

$$
\bar{h}_{c c}=\frac{t_{0}}{\tau} \bar{h}_{c c_{t<t_{0}}}+\left(1-\frac{t_{0}}{\tau}\right) \bar{h}_{c c_{t>t_{0}}}
$$

In Equation 2.18, $\bar{h}_{c c_{t<t_{0}}}$ comes from Equation 2.16, with $t_{0}$ substituted for every occurrence of $\tau$ and $\bar{h}_{c c_{t>t_{0}}}$ comes from Equation 2.17. After the particle convection heat transfer has been evaluated using Equation 2.18, this value is used in Equation 2.13 along with Equation 2.14 to obtain the overall heat transfer coefficient.

\section{Significance of the Masoumifard Correlation}

Masoumifard et al. (2008) noted that in beds of small particles, operating at or near atmospheric pressure and at relatively low temperature, particle convection is the most significant mechanism of heat transfer. For this purpose their work was primarily concerned with particle convection. In their later study which was focused on finding the maximum heat transfer coefficient, they observed that it occurs when $\tau=t_{0}$ (Masoumifard et al., 2010). In their work, $\tau$ is dependent on particle diameter, minimum fluidizing velocity, the ratio of current and minimum fluidizing velocity and the ratio of particle diameter to immersed surface diameter. From Figure 2.5, the impact of all of these parameters can be seen, but it is also 
evident that any sort of motion of the immersed surface would also alter $\tau$, although the exact contribution is not clear because the motion is perpendicular to the fluidizing velocity.

\subsection{Summary}

\subsubsection{Fluidization}

Fluidization is a phenomena where a collection of solid particles exhibit fluid-like behavior and motion. It occurs when fluid with sufficient mass flow and velocity flows upward through the particles, suspending them and forcing the bed to expand. In order to achieve this, the pressure drop must first exceed the weight of the particles per bed area, so that the interparticle forces are broken. This phenomenon is known as incipient fluidization. If the fluid flow continues to increase, in gas fluidized beds, bubbles begin to form shortly after, with their size and velocity being dependent on the particle properties. Additional increases can lead to particles being carried out of the bed completely.

The nature of fluidization is highly dependent on the particles, which is why Geldart (1973) classified them into four categories; C, A, B and D, by order of increasing size. Group C particles are very cohesive and difficult to fluidize, Group A are small, have high bubble velocity and solids mixing, Group B are the easiest to fluidize and have a moderate bubble velocity and solids mixing, while Group D are somewhat difficult to fluidize, have a bubble velocity that is lower than the fluid velocity and have minimal solids mixing.

Since incipient fluidization occurs when the pressure drop across the bed is equal to the particle weight per bed area, the point at which it occurs can be determined. The Ergun equation (Equation 2.4), shows the proportionality between the fluid velocity and the pressure gradient in the expanding region of a fluidized bed. It consists of a laminar and turbulent term, each of which grows to dominate the equation within their respective flow regimes. In laminar flow conditions $\left(\operatorname{Re}_{p}<1\right)$, the turbulent term of the Ergun equation 
is negligible, and it becomes the Carman-Kozeny equation (Equation 2.5). The velocity at incipient fluidization is called Minimum Fluidization Velocity $U_{m f}$, and can be found using the Ergun Equation if the pressure at the bed inlet, and the mean bed pressure are set as equal. When rearranged and isolated for $U_{m f}$, it becomes Equation 2.9. As can be seen from Equation 2.9, the key parameters governing $U_{m f}$ are the dimensionless Archimedes number, gas viscosity, gas density and particle diameter.

\subsubsection{Heat Transfer}

Fluidized beds have excellent temperature uniformity due to high solids mixing and high heat transfer coefficients, on the order of 250-700 W/ $\mathrm{m}^{2} K$ (Gupta and Sathiyamoorthy, 1998), making them an excellent heat transfer medium. There are three main modes in which heat transfer to immersed surfaces in fluidized beds occurs, namely: particle convection, gas convection and radiation. For simplicity, these three modes can be regarded independently and summed to determine an overall heat transfer rate.

In beds filled with small Group A particles, particle convection dominates, while in beds of large Group D particles it is gas convection that is the primary contributor. Group B particles, which fall in between $\mathrm{A}$ and $\mathrm{D}$, rely on both particle and gas convection, and show varied behaviors based on fluid velocity and particle diameter. Typically in beds of Group B particles, the heat transfer rate is inversely proportional to the particle diameter, however the influence of gas velocity is more complex. Since they rely on both particle and gas convection, Group B beds experience a maximum at an intermediate point where the sum of the particle and gas convection is the highest.

Radiation plays an important role in heat transfer at temperatures above $675 \mathrm{~K}$. Despite its nonlinear dependence on temperature, it is possible to consider the radiative component separately, and add it to the particle and gas convective ones at the end. 


\subsubsection{Correlations}

The Grewal and Saxena correlation, Equation 2.10 (Grewal and Saxena, 1980), is the most popular correlation for predicting the heat transfer to cylinders immersed in fluidized beds. It owes its popularity to the fact that it is the only correlation that can predict the heat transfer to medium and large diameter cylinders within a range of $\pm 25 \%$ (Saxena, 1979).

A more recent study by Friedman et al. (2006), has shown that the heat transfer to small and large tubes exhibit different trends and cannot be effectively predicted with a single correlation. Friedman's data collected on cylinders between 1-9 $\mathrm{mm}$ in diameter could not be predicted by the Grewal and Saxena correlation, nor any of the other previously existing ones. Friedman's own correlation, Equation 2.12, shows that unlike their large counterparts, small diameter cylinders do not show an increase in heat transfer rate past $2.5 \times U_{m f}$.

Masoumifard et al. (2008) studied particle convection in more detail and developed a correlation for predicting heat transfer based on the cluster-renewal method. This correlation and their later investigation of the maximum heat transfer coefficient illustrates the importance of the contact time between the particles and the immersed surface, $\tau$, to the overall heat transfer. From Figure 2.5 it can be seen that movement of the immersed surface will alter $\tau$, thus altering the overall heat transfer coefficient. 


\section{CHAPTER 3}

\section{Experimental Apparatus}

\subsection{Furnace and Bed}

\subsubsection{Fluidized Bed}

The fluidized bed used in these experiments is a pilot-scale fluidized bed furnace provided by the ICE Group, with a reducer section installed on it to enable operation at higher fluidizing velocities. The furnace has a plenum that is $1270 \mathrm{~mm}$ long, $610 \mathrm{~mm}$ wide and $152 \mathrm{~mm}$ deep, leading to three porous hearth tiles with a total surface area of $940 \mathrm{~mm}$ by $457 \mathrm{~mm}$. The reducer has a $965 \mathrm{~mm}$ by $152 \mathrm{~mm}$ cross section, reducing the flow area from $0.43 \mathrm{~m}^{2}$ to $0.147 \mathrm{~m}^{2}$, and a height of $470 \mathrm{~mm}$. It is constructed from particle board due to the low temperature nature of the experiments, and is sealed to the furnace using a combination of closed cell weather stripping and heavy duty silicone. In order to prevent the reducer from being lifted by the fluidizing air and damaging the seal, an H-frame, constructed from square steel tubing, sits on top of the reducer to maintain pressure and is bolted directly into the floor of the furnace. The frame is reinforced by a steel strip with $12 \mathrm{~mm}$ by $6.5 \mathrm{~mm}$ cross section welded to the top to prevent any bending in the vertical direction and apply even pressure all throughout.

Two different particle configurations are utilized, both consisting of two layers of particles: a coarse lower layer and a fine upper; both made of aluminum oxide. In the first configuration, the coarse layer has a depth of approximately $65 \mathrm{~mm}$ and is composed of particles of 1000 $\mu m$ mean diameter, which are not fluidized, and the fine layer is approximately $356 \mathrm{~mm}$ in depth and is composed of $250 \mu \mathrm{m}$ mean particle diameter (MPD) particles that are fluidized. In the second configuration, the bottom coarse layer extends to a depth of $267 \mathrm{~mm}$ and is 


\subsection{Air Delivery and Heating System}

composed of the same $1000 \mu \mathrm{m}$ MPD particles, but the upper fine layer is made up of $204 \mu \mathrm{m}$ particles and has a depth of $154 \mathrm{~mm}$. In both configurations there is a $9.5 \mathrm{~mm}$ horizontally centered hole in the bed container, located $300 \mathrm{~mm}$ above the distributor to allow the wire to be fed through. There are adjustable apertures made of thin sheet metal on the inside and outside of the bed walls, and the hole is filled with low density foam to prevent air leakage and minimize the blowing effect on the wire outside of the bed. A cross section of the fluidized bed as well as details of the mounting and wire aperture can be seen in Figure 3.1.

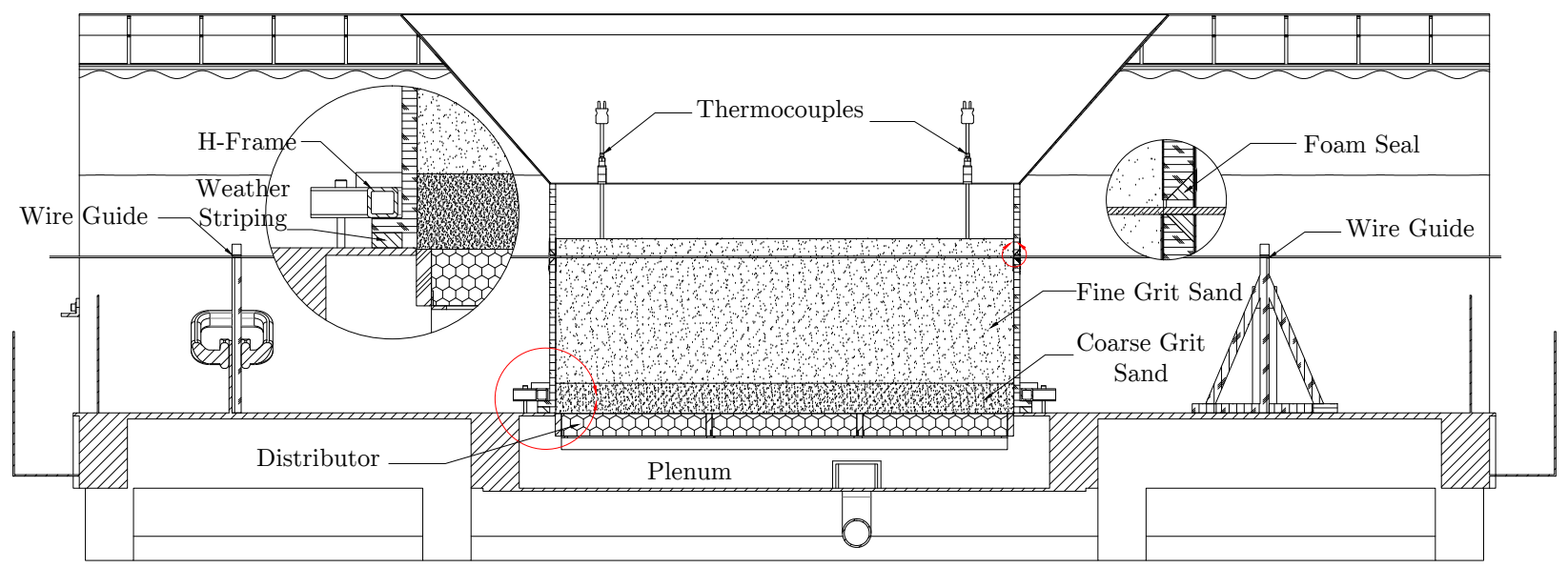

Figure 3.1: Cross section of fluidized bed

\subsection{Air Delivery and Heating System}

\subsubsection{Air Delivery System}

Fluidizing air is supplied by a Spencer multi-stage centrifugal type 3005\#2 blower driven by a $5 \mathrm{hp}, 3500 \mathrm{rpm}$ electric motor, which is capable of delivering $229.4 \mathrm{~m}^{3} / \mathrm{hr}$ of air at 122 $\mathrm{kPa}$. The air leaves the blower through a $76.2 \mathrm{~mm}$ PVC pipe, is later reduced to a $50.8 \mathrm{~mm}$ diameter at which point there is a Flow Products venturi flow meter installed. There is a 


\subsection{Wire Movement System}

Dwyer Instruments manometer connected to it to keep track of the mass flow which allows the fluidizing velocity to be calculated. After the flow meter there is a ball valve present which is used for controlling the air flow and a pair of Wika $34.5 \mathrm{kPa}$ pressure gauges to monitor the pressure drop across the heater, which will be discussed in the next section.

\subsubsection{Heating System}

The heater is a Chromalox ADHT-015FV unit, installed in a custom enclosure that was fabricated by the department's technical staff. It is insulated in order to prevent heat loss to the surroundings, as well as for operator safety. Heater control is handled by a wall mounted control panel. The control system operates based on two parameters, a desired bed temperature, and a maximum allowable heater temperature, both of which can be set using the wall mounted panel. Additionally, the panel is capable of monitoring a third temperature reading, which does not affect the operation in any way, but is simply present to supply additional information to the operator.

The air delivery and heating system can be seen schematically in Figure 3.2.

\subsection{Wire Movement System}

The wire movement system consists of two parts, a pulling side that pulls the wire through the bed and collects it, and a payoff side which is where the wire resides prior to the experiment. On the pulling side, there are two spools, each powered by a Pacesetter 48R-5H 0.75hp motor and controlled by a TECO JNEV-201-H3 AC drive. The motors have a built in gearbox and are capable of moving the wire up to a maximum speed of $120 \mathrm{~m} / \mathrm{min}$ with the current spools. The spools have a diameter of $406 \mathrm{~mm}$ and are mounted on a $1530 \mathrm{~mm}$ by 1212 $\mathrm{mm}$ by $483 \mathrm{~mm}$ steel frame. The frame is also host to three guiding and tensioning pulleys, as well as an AMACOIL AKI3-15-6 traversing unit to control the take up of the wire. The traversing unit is powered via a belt and pulley system from the second motor and spool 


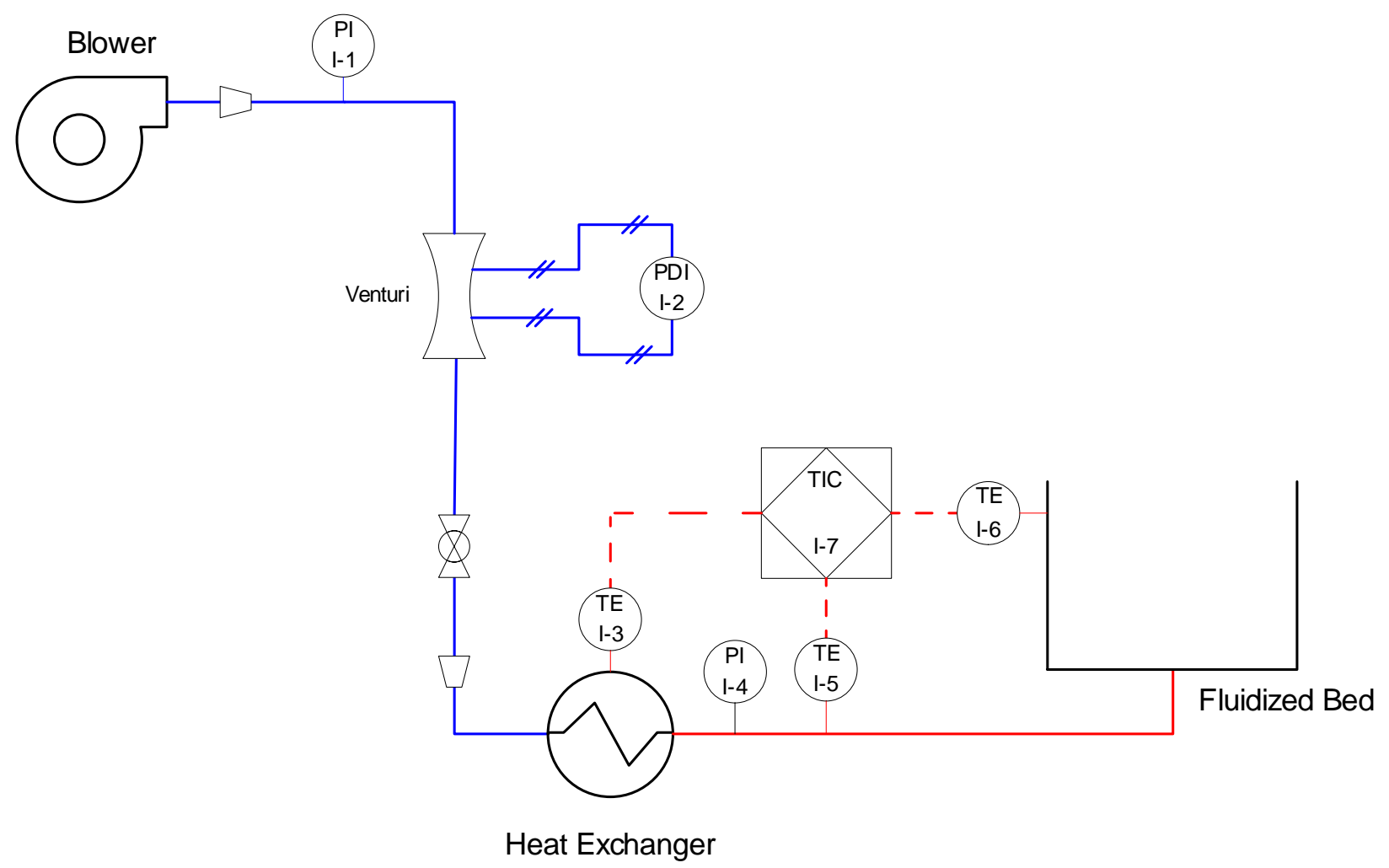

Figure 3.2: Air delivery and heating system

combination, which acts as the collecting spool. On the driving spool, there is a Shimpo DT105A tachometer to monitor the wire speed. The motor on the driving spool is configured to provide a constant speed, while the one on the collecting spool is configured to provide a constant torque.

On the payoff side, there is a single $406 \mathrm{~mm}$ diameter spool with a friction brake and hand crank for rewinding. The purpose of the friction brake is two fold, as it is meant to provide back tension to the wire so that it does not sag, as well as to prevent the spool from freewheeling from inertia when the system stops. There is also a Cometo AS574 single plane wire straightener and two guide pulleys to control the entry into the wire straightener. The wire movement system is featured in Figures 3.3 and 3.4 . 


\subsection{Instrumentation}

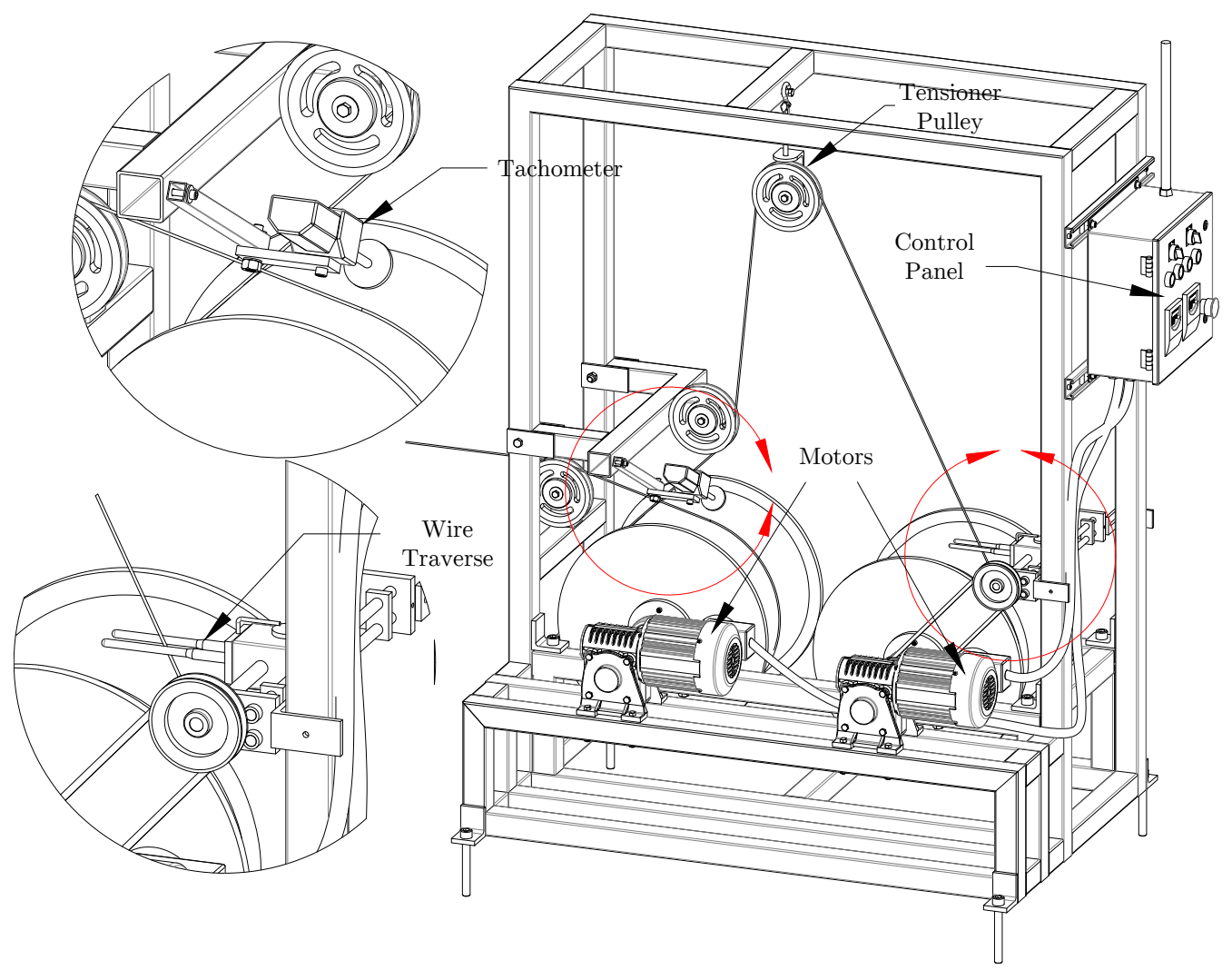

Figure 3.3: Pulling side of wire movement system

\subsection{Instrumentation}

In order to successfully determine the heat transfer to the wire, several parameters need to be known. The wire temperature at the bed inlet and outlet is of course needed, as well as the bed temperature itself. Additionally, since the wire speed is the parameter being investigated, it should also be known. Finally, the fluidizing velocity needs to be known so that the data can be compared with other studies. 


\subsection{Instrumentation}

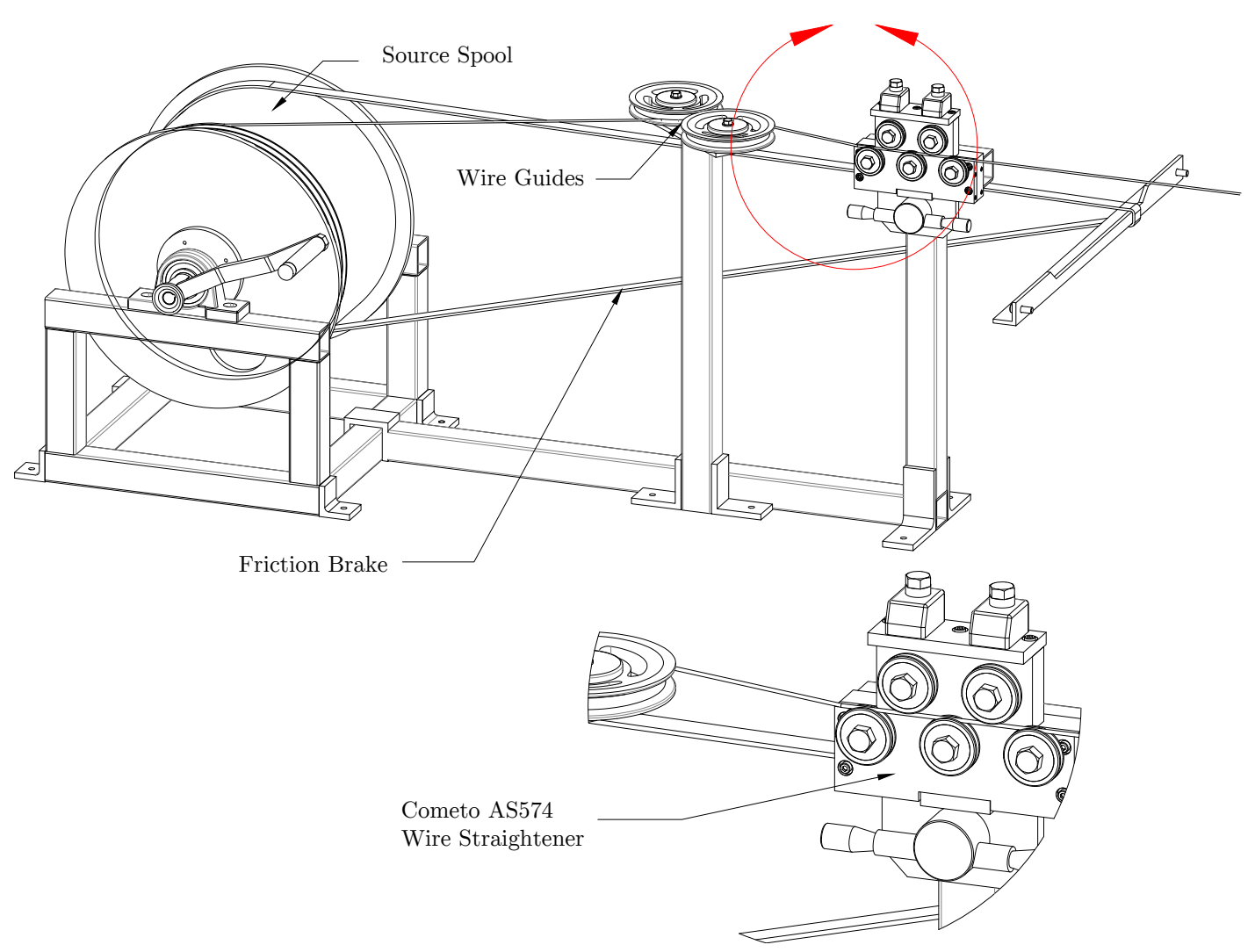

Figure 3.4: Feeding side of wire movement system

\subsubsection{Temperature Measurement}

The inlet and outlet temperatures are monitored by two Anritsu MW-44K-TC2-ANP temperature probes, connected to a Data Translation DT9828 Data Acquisition Unit (DAQ), which is capable of accepting up to 8 thermocouples and offers a USB computer connection for data logging. There is also an immersion type $\mathrm{K}$ thermocouple connected to the same DAQ for monitoring the bed temperature. The entirety of the wire movement system along with the fluidized bed and temperature measurement system can be seen in Figures 3.5 and 3.6. 


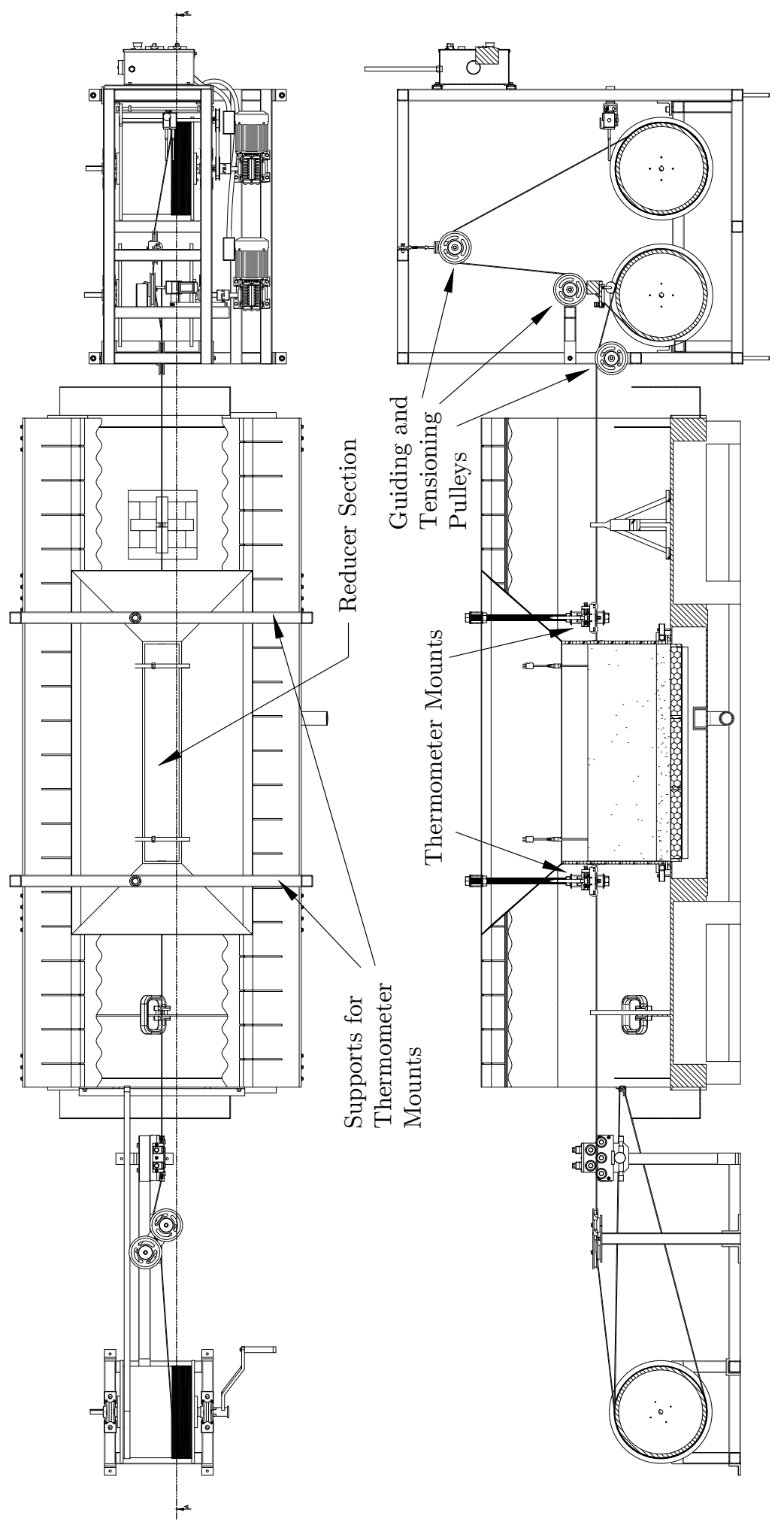




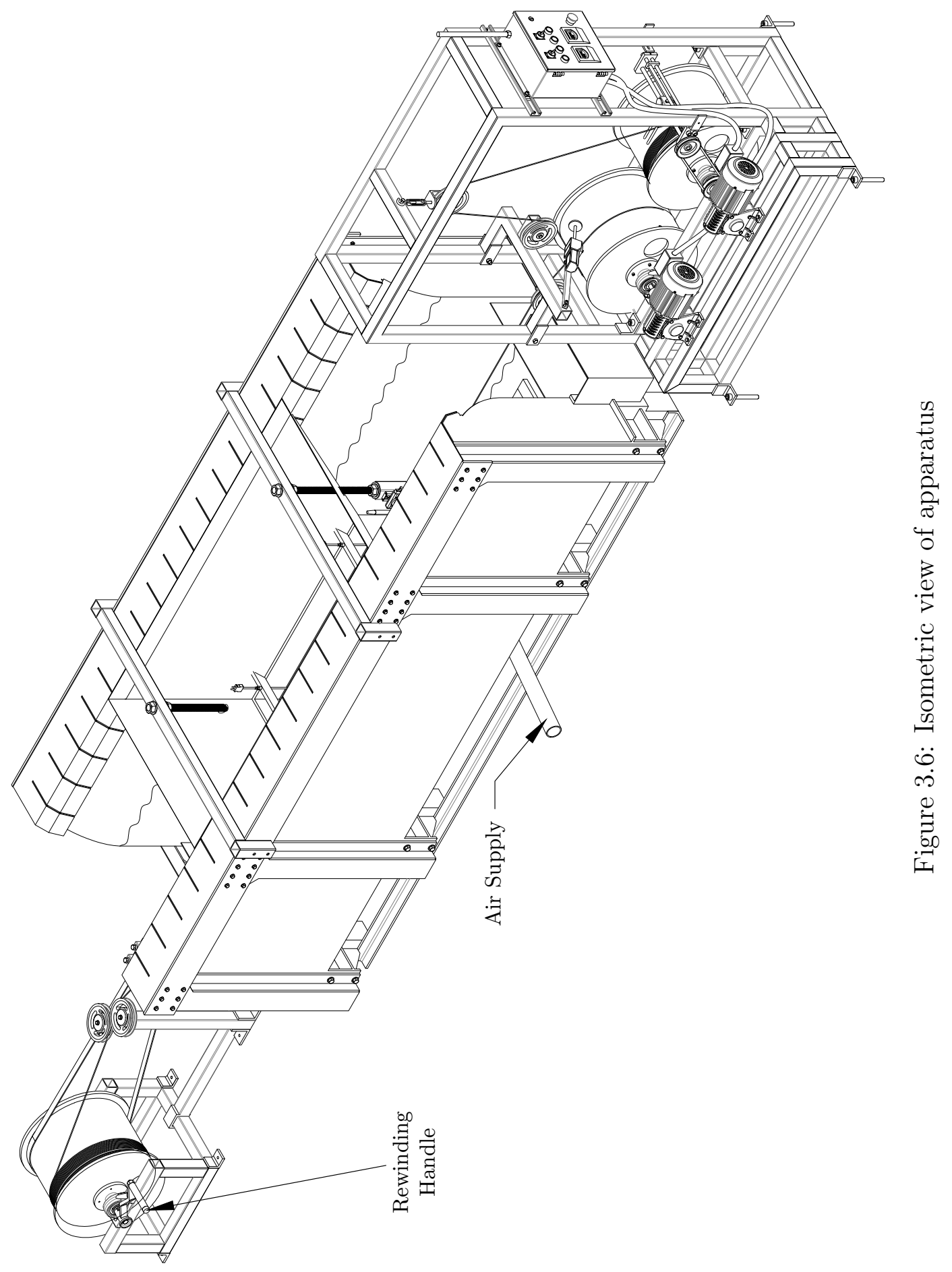




\subsubsection{Data Acquisition}

\section{Hardware}

The Data Translation DT9828 used in these experiments has two sides with screwdown terminals for both digital and analog type inputs. It is capable of acquiring data at a maximum frequency $600 \mathrm{~Hz}$, and is accurate to $\pm 0.09^{\circ} \mathrm{C}$. It also exhibits a maximum noise level of $\pm 0.1^{\circ} \mathrm{C}($ DataTranslation, 2014a). On the analog side, there are 16 channels, which are reduced to 8 when differential operation is required, such as in the case of a thermocouple. To connect a thermocouple, first a channel should be selected. In this instance, the thermocouple monitoring the bed temperature is connected to Channel 0, the bed inlet to Channel 4 and the outlet to Channel 7. This was done to provide adequate spacing between the connections and minimize noise. Details of the unit and the connected thermocouples can be seen in Figure 3.7.

The DAQ itself needs to be connected to the computer that has the QuickDAQ software and appropriate device drivers installed, via a USB 2.0 port. If a USB 2.0 port is not present, a 3.0 port can be substituted, but it is not recommended that any older versions of USB be utilized. The process of installing the software and drivers can be found in the instruction manual in DataTranslation (2014b). The calibration of the DAQ and thermocouples will be discussed in a later section.

\section{Software}

The software used for data acquisition is QuickDAQ, which is supplied with the DT9828. It is capable of logging and displaying data live at up to $200 \mathrm{~Hz}$. Additionally, QuickDAQ can export data to a CSV file which is readable by programs such as Microsoft Excel and Mathworks Matlab. 


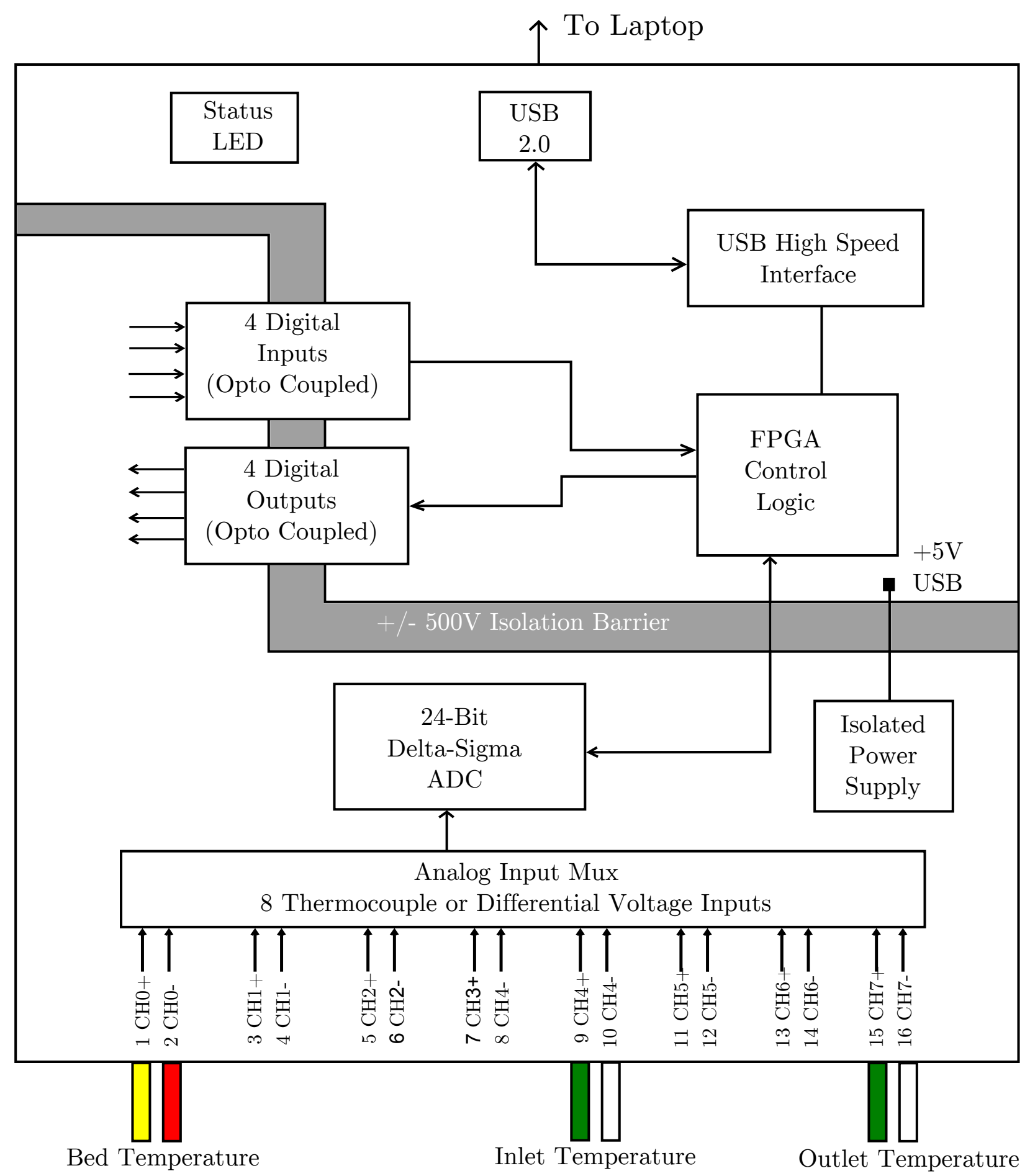

Figure 3.7: DT9828 data acquisition unit and connections 


\subsubsection{Velocity Measurement}

Velocity measurement and tracking is handled by a Shimpo DT-105A Tachometer. The unit is accurate to $\pm 0.06 \mathrm{rpm}$ between 0.10-999.9 rpm and $\pm 0.6 \mathrm{rpm}$ between 1000.0-9999.9 rpm, $\pm 0.006 \%$ of the reading, or \pm 1 digit, whichever is largest. In this experimental configuration, the tachometer would encounter rpm in the range of 60-500. The tachometer is also capable of taking measurements in different units, such as $\mathrm{m} / \mathrm{min}$ which is the desired one in this case. When configured in $\mathrm{m} / \mathrm{min}$, the tachometer measures the linear surface speed of the drum directly, rather than rpm, as the diameter of the tachometer's wheel is included in its programming.

The DT-105A is not capable of outputting data to an external source such as a data logger, it is only capable of displaying the live value and retaining certain information in memory. Unfortunately, it cannot average the data from each run, but it can store a maximum and minimum value and from that an average value can be obtained. For the purposes of this study it is assumed that the average value lies exactly half way between the maximum and minimum value.

\subsubsection{Flow Measurement}

The Flow Products venturi flow meter is installed in-line before the ball valve that regulates the air flow to the bed. The venturi produces a pressure drop of $378.46 \mathrm{~mm}$ of water at a flow rate of $254.85 \mathrm{~m}^{3} / \mathrm{hr}$ and the current flow rate can be calculated using the measured pressure drop at the manometer. The manometer has a maximum reading of $914.4 \mathrm{~mm}$ of water, which corresponds to a flow rate that exceeds the maximum blower capacity and as such is well suited to its purpose. 


\subsection{Startup and Shutdown Procedure}

\subsubsection{Startup}

Each subsystem is controlled by a separate control panel, so there are three panels present, one for fluidizing air control, one for heater control and one for wire motion control. Full system startup should begin with the fluidizing air. To turn on the fluidizing blower, the appropriate panel needs to be energized first, using its power toggle, located on the panel itself. Prior to starting the fluidizing blower, hearing protection is fitted, as the starting procedure is extremely loud and can be harmful to human hearing. After proper hearing protection is fitted, the blower can be started by depressing the green button labeled 'Fluidization'. After the blower has started, a visual inspection is performed of the fluidized bed to ensure the fluidizing velocity is appropriate, and the ball valve is adjusted accordingly. Finer adjustment is left for a later point, when the bed has reached steady state temperature.

After the fluidizing bed is running, the heater can be turned on. This is done by first energizing the heater control panel via the toggle mounted on it. When power is supplied to the heater control panel, the temperature settings become visible. In order from left to right, they are the outlet temperature of the heat exchanger, the heater core temperature, and the bed temperature. The first value is simply a reading and has no impact on operation, but the second two are examined to ensure that the correct maximum heater temperature, and target bed temperature are set. This is done by pressing the 'Set' button on each respective temperature display, and using the up and down arrows to reach the desired temperature, then accepting it using the 'Set' button again. When the settings have been verified, the heater can be started by depressing the green button labeled 'Heater'. It is important to note that reaching the operating temperature of the fluidized bed can take several hours, as the system has very high thermal mass. Over the course of this process, the maximum heater temperature setting may trigger several times, causing the heater to temporarily shut off. The correct way to proceed is by waiting until the heater core temperature drops to below 
$150{ }^{\circ} \mathrm{C}$, and then restarting it by depressing the green button again. Future modifications should automate this process.

The wire movement system should not be started until the fluidized bed has reached steady state temperature, as the changing temperature will affect the validity of the results. Once it has, the starting procedure begins by moving the large wall toggle to the on position to power up the transformer which in turn powers the control panel. Then the main panel power switch on the right side is turned to the on position. Each controller has its own power toggle as well, and these should both be set to the on position as well. Finally, both green buttons are depressed to turn on the controllers. It is important to note, that this does not cause the wire to start moving, but merely powers up the controllers. Prior to starting the wire movement, it is advised to inspect the entire wire path, and ensure that nothing is caught or tangled. When all of this is completed, the wire movement can be started using the small 'Start/Stop' button on each controller. The collection spool is started first, to put tension into the entire system, and then the driving spool can be started.

A flowchart of this process can be seen in Figure 3.8. The layout of the various connections can be seen in Figure 3.9.

Data collection can begin either immediately before or after the wire movement system is engaged, as all data prior to steady state is discarded. To commence data collection, it is sufficient to press the circular 'Record' button inside the QuickDAQ software.

\subsubsection{Shutdown}

The fluidization and wire drive systems are independent of each other and the order of shut down is not relevant, although it is recommended to shut down the wire movement system first in order to minimize the rewinding time. Data logging can and should be stopped first, as soon as all of the necessary data has been collected. Despite safeguards built into the heater to prevent damage to the system and operator injury, it is still highly advisable that the heater be stopped before the fluidizing air, giving the fluidizing air an opportunity to 


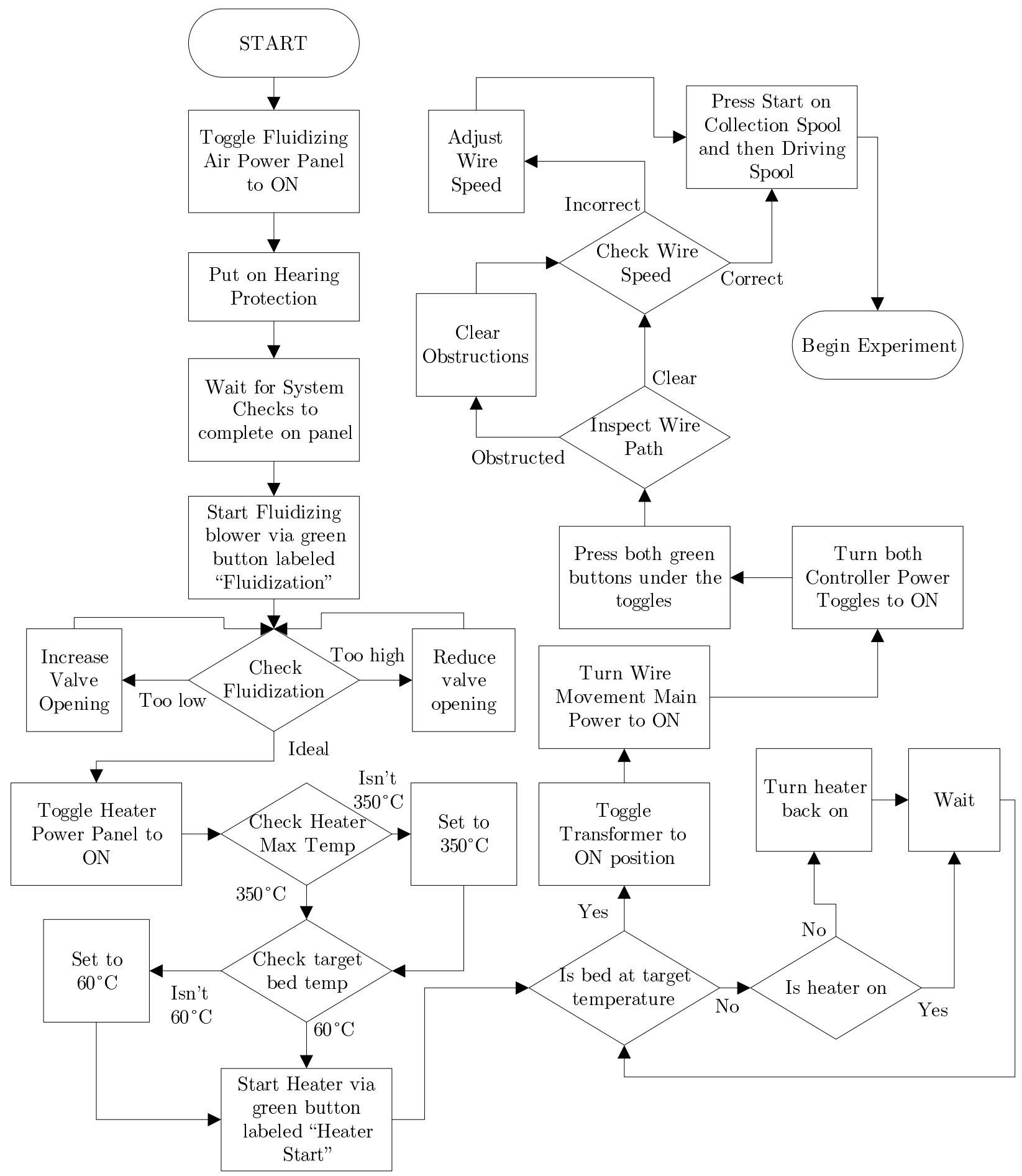

Figure 3.8: System startup procedure 


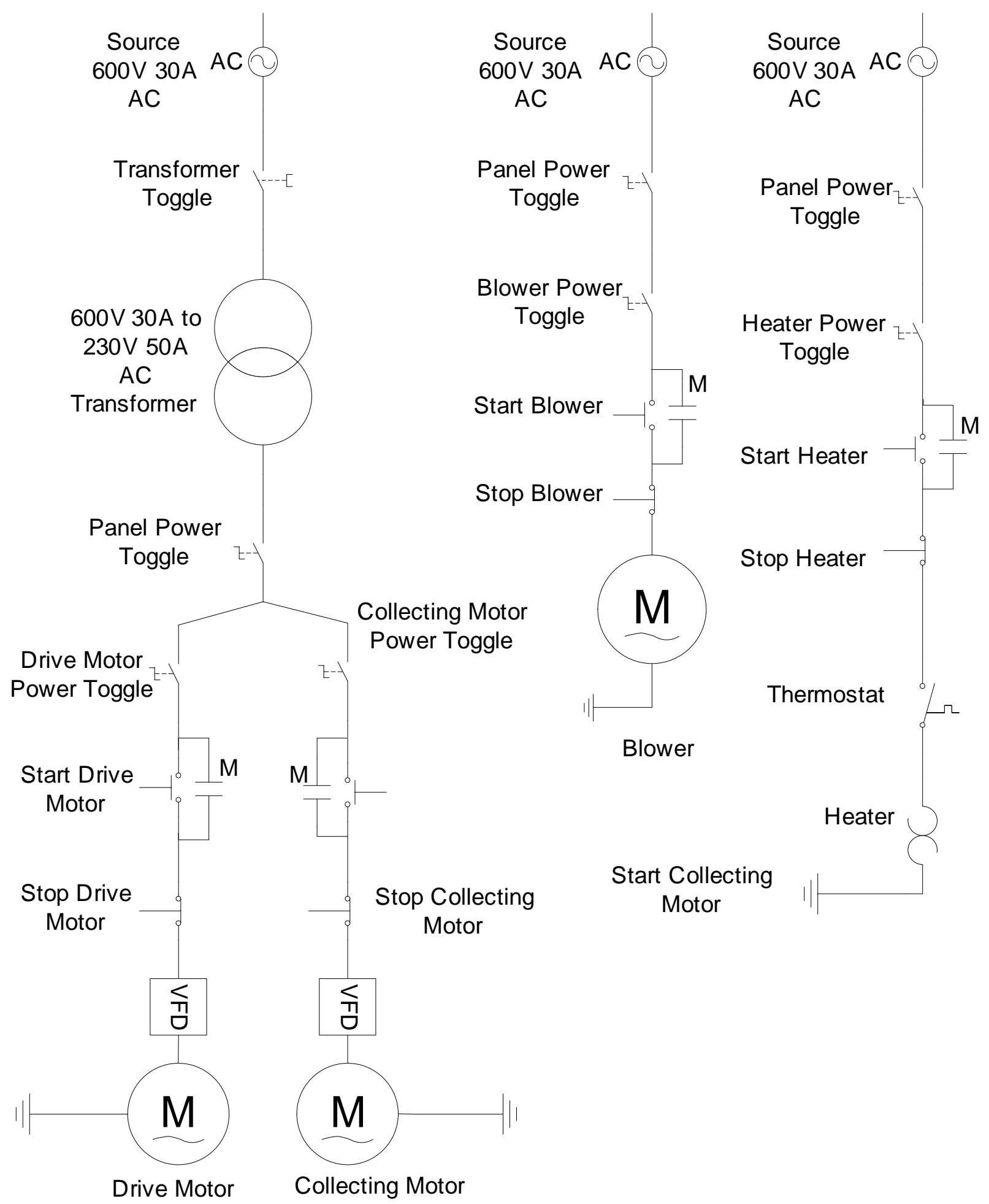

Figure 3.9: Wiring of various systems 


\subsection{Limitations}

cool the heater for some time before shutoff. This is done by depressing the red "stop" button labeled 'Heater' which is just below the green one used to turn it on. Main power to the panel is left on so that the temperature can be monitored. The fluidizing blower is left on, until the heat exchanger temperature drops to under $100^{\circ} \mathrm{C}$, at which point the heat exchanger controller can be turned off, and the panel de-energized. At this point, the fluidization blower can be stopped as well, and that panel de-energized in the same manner that it was turned on.

To properly stop the drive system, first the start stop button on the controllers must be pressed, starting with the drive controller, and then the collection one. After that the red stop buttons are depressed on both, and the toggles are toggled to off. The main toggle on the panel is toggled to off as well, and finally the transformer power is toggled to the off position.

\subsection{Limitations}

The main limitation of this system is that it is restricted to low temperature operation. The fluidized bed itself is constructed from particle board, which is a flammable material and as such it is ill advised to operate the system at temperatures exceeding $100^{\circ} \mathrm{C}$. Even without this material limitation, an electric heater is used instead of combustion, so truly high temperature operation $\left(>700^{\circ} \mathrm{C}\right)$ is impossible. This can alter the results somewhat, as in high temperature operation there are significant radiation effects, which would not appear at the lower temperatures to which the apparatus is limited.

The blower is capable of providing upwards of $229.4 \mathrm{~m}^{3} / \mathrm{hr}$, which has been found sufficient to fluidize the bed up to nearly $6 \times U_{m f}$ for the 60 grit particles, and even higher for the $70 \& 80$ grit mix. Based on a previous study by Friedman (2006), the heat transfer coefficient does not appreciably increase after $2.5 \times U_{m f}$, and as such the mass flow provided by the blower is more than adequate. 
The wire straightener and thermometers are designed to accept wire in the 1-7 $\mathrm{mm}$ diameter range and would need to be changed if a different size material is to be used. For the purposes of this study, that range covers the wire diameters of interest and the apparatus is satisfactory in that regard.

Finally, the motors and controllers are capable of moving the wire at a maximum speed of $120 \mathrm{~m} / \mathrm{min}$. This covers typical industry usage speeds quite well, and does not reduce the apparatus' usability in any way.

\subsection{Summary}

\subsubsection{Fluidized Bed}

The bed is a pilot scale fluidized bed furnace with a reducer section installed to allow it to operate at higher fluidizing velocities. The reducer is fabricated out of particle board and sealed to the furnace floor with closed cell weather striping and heavy duty silicone. There is an additional brace made from square steel tube to ensure the fluidizing air does not lift the reducer and damage the seal.

There are two different bed configurations, both involving a coarse lower layer of $1000 \mu \mathrm{m}$ diameter Aluminum Oxide sand. In the first configuration this layer is only $65 \mathrm{~mm}$ high, while in the second it extends to $267 \mathrm{~mm}$. The key difference between the two configurations is the upper layer of sand, which is $356 \mathrm{~mm}$ of $250 \mu \mathrm{m}$ Aluminum Oxide sand for the first, and $154 \mathrm{~mm}$ of $204 \mu \mathrm{m}$ for the second. In both configurations, the wire passes through a $9.5 \mathrm{~mm}$ hole, $300 \mathrm{~mm}$ above the distributor which is filled with low density foam to prevent leakage. The details of the bed can be seen in Figure 3.1.

The fluidizing air is supplied by a Spencer type 3005\#2 blower, driven by a $5 \mathrm{hp}, 3500 \mathrm{rpm}$ motor and heated by a Chromalox ADHT-015FV heater in a custom enclosure. Metering is handled by a combination of Flow Products venturi flow meter and a Dwyer Instruments 
manometer, and the flow rate is controlled via a ball valve. The heater has a thermostat which functions based on two parameters, a target bed temperature, and a maximum allowable heater temperature. More details of the system can be seen in Figure 3.2.

\subsubsection{Wire Movement System}

The system is made up of two parts, the pulling and the payoff sides. The pulling side consists of a large frame made of square steel tube that is home to two spools, each driven by Pacesetter 48R-5H $0.75 h p$ motors and controlled by TECO JNEV-201-H3 AC drives. There are also three guiding and tensioning pulleys, as well as an AMACOIL AKI3-15-6 traversing unit and a Shimpo DT-105A tachometer to monitor the wire speed. The pulling system is featured in Figure 3.3.

The payoff side has a single spool with a friction brake on it to give appropriate wire tension and ensure it does not freewheel from inertia when the system stops. There is also a Cometo AS574 single plane wire straightener and two guide pulleys to control the entry into the wire straightener. The wire payoff system is featured in Figure 3.4.

\subsubsection{Instrumentation and Data Acquisition}

The parameters of interest in this study are the wire temperature at the bed inlet and outlet, the bed temperature, the wire speed and the fluidizing velocity. There are three systems at work to monitor these parameters.

The temperature of the wire is monitored with two Anritsu MW-44K-TC2-ANP moving wire probes, while the bed temperature is monitored by a standard immersion type thermocouple. These three instruments are connected to a Data Translation DT9828 DAQ, which obtains and transmits the values to a computer for logging. The DAQ and temperature probe connections are featured in Figure 3.7. 
The wire speed is monitored by a Shimpo DT-105A tachometer, which is capable of displaying the live wire speed in $\mathrm{m} / \mathrm{min}$ as well as storing the maximum and minimum speeds per run, but not capable of computer based data logging.

Finally, the fluidizing velocity is determined by measuring the flow with a Flow Products venturi, connected to a Dwyer Instruments manometer. With the mass flow and cross sectional area known, it is possible to determine the air velocity.

To ensure the system operates safely and effectively, there is an outlined procedure for starting and stopping it. The starting procedure is featured in Figure 3.8. When stopping, it is recommended that the wire movement system be halted first, to minimize rewinding, followed by the heater, and finally the fluidizing air when the heater core temperature is less than $100^{\circ} \mathrm{C}$. The stopping procedure for each system is simply the reverse of the starting. 
CHAPTER 4

\section{Experimental Procedure}

\subsection{Apparatus Setup}

The bed was used in two different configurations, one with 60 grit, $250 \mu \mathrm{m}$ mean particle size and one with a mixture of 70 and 80 grit particles, with an average diameter of $204 \mu \mathrm{m}$. In both configurations, the bottom of the bed was filled with coarse 8 grit particles of $\mathrm{Al}_{2} \mathrm{O}_{3}$ sand with a mean diameter of $2210 \mu \mathrm{m}$. The coarse sand serves many purposes, such as to act as a barrier between the distributor and the fluidizing sand so that wear and damage to the distributor is avoided, to improve the quality of the air distribution, and to keep the fluidizing sand away from the seals so that leaks do not form. Additionally, in circumstances when the supply of fluidizing sand is limited, coarse sand can be used to fill in some height and allow less fluidizing sand to be used. Studies by Masoumifard et al. (2008) and many others have shown that heat transfer does not vary with bed height, so the fluidized layer can be safely reduced without any adverse effects.

In the first configuration, the coarse layer was only $65 \mathrm{~mm}$ in depth, and the other $356 \mathrm{~mm}$ were filled in with the fine 60 grit, $250 \mu \mathrm{m}$ sand for fluidization. For the second, due to a shortage of fine sand, the coarse layer extended to a depth of $267 \mathrm{~mm}$ and a mixture of 70 and 80 grit sand with an average diameter of $204 \mu \mathrm{m}$ was filled over top of it for an additional $154 \mathrm{~mm}$ of depth. In order to ensure an even distribution of fine particles, equal parts 70 and 80 grit sand were scooped in alternating order and deposited into a larger container, where they were mixed before adding them to the bed. Additionally, the particle mix was fluidized for at least four hours prior to conducting any experiment, as that is the time required for it to reach operating temperature, so it is safe to assume that they are a uniform mixture. The average particle diameters in all cases were determined using microscopic imaging particle analysis by the Abrasive Machining Laboratory at Ryerson University to ensure accuracy as $U_{m f}$ is highly dependent on particle diameter. 


\subsection{Calculation of the Heat Transfer Coefficient}

The wire used for all experiments was 1188 H18 Aluminum wire, composed of 99.88\% pure Aluminum. It had a diameter of $2.8 \mathrm{~mm}$ and was approximately $550 \mathrm{~m}$ in length allowing quite a few tests to be carried out on a single wind. Due to the high purity of its composition, reference values for Aluminum were used for all physical and thermal properties.

The apparatus functioned fairly well in the initial tests, but further testing revealed some issues that required correction. Wire that is kept under tension has a tendency to vibrate and oscillate if agitated and the constant bubbling of the fluidized bed was causing the wire to temporarily lose contact with the thermometers, leading to fluctuations in temperature readings. Also, due to the fact that rewinding was done manually, the wire was not perfectly wound onto the payoff spool, causing the occasional short spike or drop in speed, which would also be reflected in the temperature data. The disturbances caused by the wire winding were rare enough that they could be eliminated from the data, but sudden changes in speed resulted in equally sudden changes in tension, which in turn caused the motors to overload and stop.

The wire oscillation issue was solved by adding additional wire guides to stabilize the wire near the thermometers so that there is very minimal unsupported length before and after each thermometer. There were still oscillations present in the system wherever there were large lengths of unsupported wire, but since they were far away from the thermometers, the quality of the data improved dramatically. The motor overload issue was solved by adding a spring to the tensioning pulley between the two spools on the pulling side, which absorbed these variations in tension and resulted in far smoother operation.

\subsection{Calculation of the Heat Transfer Coefficient}

The heat transfer coefficient, which is the parameter of interest for this study, is not a directly observable and measurable quantity such as temperature, pressure or length, it must be calculated from other parameters. 


\subsection{Calculation of the Heat Transfer Coefficient}

An expression for heat transfer into the wire can be developed by studying a control volume formed around it and performing an energy balance on it. The analysis is shown in Figure 4.1. Before proceeding, certain variables need to be defined.

$$
\begin{aligned}
\rho_{w} & =\text { Density of the wire }\left(\frac{k g}{m^{3}}\right) \\
u_{w} & =\text { Wire speed }\left(\frac{m}{s}\right) \\
C_{p, w} & =\text { Specific heat capacity }\left(\frac{J}{k g K}\right) \\
A_{c} & =\text { Wire cross sectional area }\left(m^{2}\right) \\
P & =\text { Wire perimeter }(m) \\
T(x) & =\text { Wire temperature at current location }\left({ }^{\circ} \mathrm{C}\right) \\
T_{\infty} & =\text { Bed Temperature }\left({ }^{\circ} \mathrm{C}\right) \\
\dot{Q}_{x} & =\text { Heat Flux at current location }\left(\frac{W}{m^{2}}\right) \\
h & =\text { Average Heat Transfer Coefficient }\left(\frac{W}{m^{2} K}\right)
\end{aligned}
$$

Since the wire is moving, there is advection into the control volume, represented by:

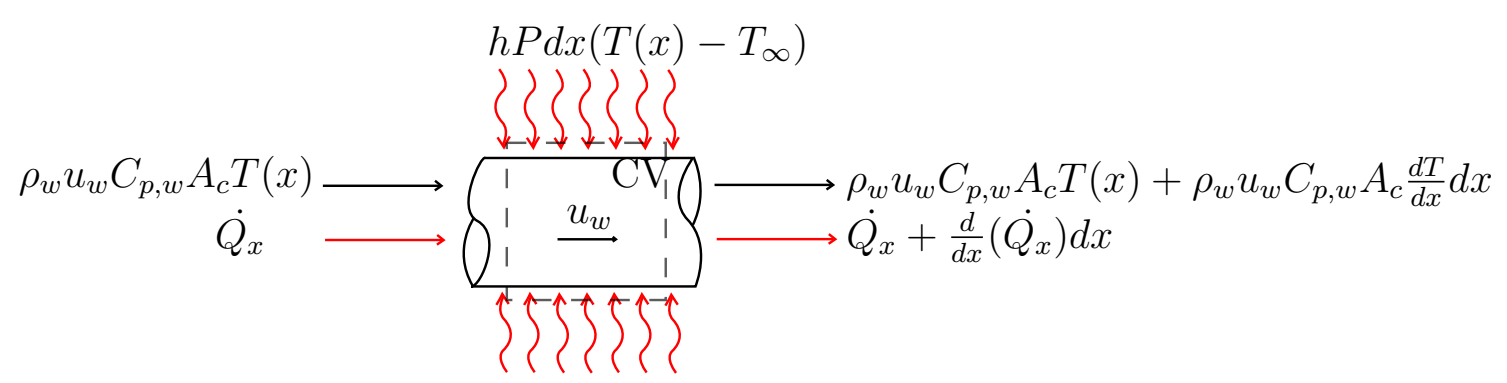

Figure 4.1: Energy balance on a control volume of wire

$$
\rho_{w} u_{w} C_{p, w} A_{c} T(x)
$$




\subsection{Calculation of the Heat Transfer Coefficient}

and advection out of it:

$$
\rho_{w} u_{w} C_{p, w} A_{c} T(x)+\rho_{w} u_{w} C_{p, w} A_{c} \frac{d T}{d x} d x
$$

There is also conduction along the wire into the control volume, represented by:

$$
\dot{Q}_{x}
$$

and out of it:

$$
\dot{Q}_{x}+\frac{d}{d x}\left(\dot{Q_{x}}\right) d x
$$

Finally, there is the convection into the wire, which contains our parameter of interest, the convection coefficient, h. It is represented by:

$$
h P d x\left(T(x)-T_{\infty}\right)
$$

The control volume as well as the energy balance can be seen more clearly in Figure 4.1.

Adding up all of these energy terms leaves us with the following:

$$
\begin{aligned}
\rho_{w} u_{w} C_{p, w} A_{c} T(x)+\dot{Q}_{x} & = \\
\rho_{w} u_{w} C_{p, w} A_{c} T(x) & +\rho_{w} u_{w} C_{p, w} A_{c} \frac{d T}{d x} d x+\dot{Q_{x}}+\frac{d}{d x}\left(\dot{Q_{x}}\right) d x+h P d x\left(T(x)-T_{\infty}\right)
\end{aligned}
$$

The terms $\rho_{w} u_{w} C_{p, w} A_{c} T(x)$ and $\dot{Q}_{x}$ can be immediately eliminated as they appear on both sides of the equation, leading to:

$$
\frac{d}{d x}\left(\dot{Q}_{x}\right) d x+\rho_{w} u_{w} C_{p, w} A_{c} \frac{d T}{d x} d x+h P d x\left(T(x)-T_{\infty}\right)=0
$$

The $d x$ term can be eliminated next, which results in:

$$
\frac{d}{d x}\left(\dot{Q}_{x}\right)+\rho_{w} u_{w} C_{p, w} A_{c} \frac{d T}{d x}+h P\left(T(x)-T_{\infty}\right)=0
$$

At this point it can be observed that $Q=-k_{w} A_{c} \frac{d T}{d x}$ with $k_{w}$ representing the thermal conductivity of the wire in $\frac{W}{m K}$. Making this substitution changes the previous expression 
to:

$$
-k_{w} A_{c} \frac{d^{2} T}{d x^{2}}+\rho_{w} u_{w} C_{p, w} A_{c} \frac{d T}{d x}+h P\left(T(x)-T_{\infty}\right)=0
$$

If $\Theta(\mathrm{x})$ is then defined as the temperature difference between the bed and wire temperatures, $T(x)-T_{\infty}$, since $T_{\infty}$ is constant, $\frac{d^{2} T}{d x^{2}}=\frac{d^{2} \Theta}{d x^{2}}$ and $\frac{d T}{d x}=\frac{d \Theta}{d x}$ therefore the equation can be further simplified to:

$$
-k_{w} A_{c} \frac{d^{2} \Theta}{d x^{2}}+\rho_{w} u_{w} C_{p, w} A_{c} \frac{d \Theta}{d x}+h P \Theta(x)=0
$$

This is the ordinary differential equation that represents the heat transfer into the wire.

Two different approaches were taken for solving Equation 4.10, an analytical and a numerical approach. Both will be discussed in the next sections.

\subsubsection{Analytical Solution}

For the analytical solution, additional substitutions were made to Equation 4.10. The entire equation was divided by $k_{w} A_{c}$ and the thermal diffusivity, $\alpha_{w}\left(\frac{m^{2}}{s}\right)$ was substituted in $\left(\alpha_{w}=\frac{k_{w}}{\rho_{w} C_{p, w}}\right)$. This resulted in the following:

$$
\frac{d^{2} \Theta}{d x^{2}}-\frac{u_{w}}{\alpha_{w}} \frac{d \Theta}{d x}-\frac{h P \Theta(x)}{k_{w} A_{c}}=0
$$

Equation 4.11 was then modified further by making the following substitutions, $K=\frac{u_{w}}{\alpha}$ and $m^{2}=\frac{h P}{k A_{c}}$.

$$
\frac{d^{2} \Theta}{d x^{2}}-K \frac{d \Theta}{d x}-m^{2} \Theta(x)=0
$$

This led to Equation 4.12, which was solved in Maple to form this general solution:

$$
\Theta(x)=C_{1} e^{\left(\frac{1}{2} K+\frac{1}{2} \sqrt{K^{2}+4 m^{2}}\right) x}+C_{2} e^{\left(\frac{1}{2} K-\frac{1}{2} \sqrt{K^{2}+4 m^{2}}\right) x}
$$

To find the specific solution, the following boundary conditions were chosen. At the inlet, the 


\subsection{Calculation of the Heat Transfer Coefficient}

wire temperature was set equal to the inlet temperature $\left(\Theta(0)=\Theta_{\text {in }}\right)$, and at the outlet, the temperature was given a constant slope, meaning it was assumed to be linear $\left(\frac{d^{2}}{d x^{2}} \Theta(L)=0\right)$. Present studies on convection to a moving rod, whether they are analytical (Roy Choudhury and Jaluria, 1994), numerical (Jaluria and Singh, 1983) or experimental (Karwe and Jaluria, 1992) have all shown that while the temperature gradient immediately after the rod enters the medium is exponential, it very quickly decays to a point where it can be considered linear and goes to zero soon after that. Due to the fact that the apparatus used in this study has an extremely high dimensionless length $(>300)$, the outlet boundary condition of constant slope was easily justified.

With the aforementioned boundary conditions, the PDE was solved using Maple, and the following solution obtained:

$$
\begin{aligned}
& \Theta(x)=-\left[\Theta i n \mathrm{e}^{1 / 2\left(\mathrm{~K}-\sqrt{\mathrm{K}^{2}+4 \mathrm{~m}^{2}}\right) \mathrm{L}}\left(-\mathrm{K} \sqrt{\mathrm{K}^{2}+4 \mathrm{~m}^{2}}+\mathrm{K}^{2}+2 \mathrm{~m}^{2}\right) \mathrm{e}^{\left(1 / 2 \mathrm{~K}+1 / 2 \sqrt{\mathrm{K}^{2}+4 \mathrm{~m}^{2}}\right) \mathrm{x}}\right] / \\
& {\left[\mathrm{e}^{1 / 2\left(\mathrm{~K}-\sqrt{\mathrm{K}^{2}+4 \mathrm{~m}^{2}}\right) \mathrm{L}} \mathrm{K} \sqrt{\mathrm{K}^{2}+4 \mathrm{~m}^{2}}-\mathrm{e}^{1 / 2\left(\mathrm{~K}-\sqrt{\mathrm{K}^{2}+4 \mathrm{~m}^{2}}\right) \mathrm{L}} \mathrm{K}^{2}-2 \mathrm{e}^{1 / 2\left(\mathrm{~K}-\sqrt{\mathrm{K}^{2}+4 \mathrm{~m}^{2}}\right) \mathrm{L}} \mathrm{m}^{2}\right.} \\
& \left.+\mathrm{e}^{1 / 2\left(\mathrm{~K}+\sqrt{\mathrm{K}^{2}+4 \mathrm{~m}^{2}}\right) \mathrm{L}} \mathrm{K}^{2}+2 \mathrm{e}^{1 / 2\left(\mathrm{~K}+\sqrt{\mathrm{K}^{2}+4 \mathrm{~m}^{2}}\right) \mathrm{L}} \mathrm{m}^{2}+\mathrm{e}^{1 / 2\left(\mathrm{~K}+\sqrt{\mathrm{K}^{2}+4 \mathrm{~m}^{2}}\right) \mathrm{L}} \mathrm{K} \sqrt{\mathrm{K}^{2}+4 \mathrm{~m}^{2}}\right] \\
& +\left[\mathrm{e}^{1 / 2\left(\mathrm{~K}+\sqrt{\mathrm{K}^{2}+4 \mathrm{~m}^{2}}\right) \mathrm{L}}\left(\mathrm{K}^{2}+2 \mathrm{~m}^{2}+\mathrm{K} \sqrt{\mathrm{K}^{2}+4 \mathrm{~m}^{2}}\right) \operatorname{\theta in} \mathrm{e}^{\left(1 / 2 \mathrm{~K}-1 / 2 \sqrt{\mathrm{K}^{2}+4 \mathrm{~m}^{2}}\right) \mathrm{x}}\right] / \\
& {\left[\mathrm{e}^{1 / 2\left(\mathrm{~K}-\sqrt{\mathrm{K}^{2}+4 \mathrm{~m}^{2}}\right) \mathrm{L}} \mathrm{K} \sqrt{\mathrm{K}^{2}+4 \mathrm{~m}^{2}}-\mathrm{e}^{1 / 2\left(\mathrm{~K}-\sqrt{\mathrm{K}^{2}+4 \mathrm{~m}^{2}}\right)} \mathrm{L} \mathrm{K}^{2}-2 \mathrm{e}^{1 / 2\left(\mathrm{~K}-\sqrt{\mathrm{K}^{2}+4 \mathrm{~m}^{2}}\right) \mathrm{L}} \mathrm{m}^{2} /\right.} \\
& \left.+\mathrm{e}^{1 / 2\left(\mathrm{~K}+\sqrt{\mathrm{K}^{2}+4 \mathrm{~m}^{2}}\right) \mathrm{L}} \mathrm{K}^{2}+2 \mathrm{e}^{1 / 2\left(\mathrm{~K}+\sqrt{\mathrm{K}^{2}+4 \mathrm{~m}^{2}}\right) \mathrm{L}} \mathrm{m}^{2}+\mathrm{e}^{1 / 2\left(\mathrm{~K}+\sqrt{\mathrm{K}^{2}+4 \mathrm{~m}^{2}}\right) \mathrm{L}} \mathrm{K} \sqrt{\mathrm{K}^{2}+4 \mathrm{~m}^{2}}\right]
\end{aligned}
$$

The parameter of interest, $\mathrm{h}$, appears implicitly inside $m^{2}\left(m^{2}=\frac{h P}{k A_{c}}\right)$, and cannot be isolated. The only way to determine it would be iteratively, however Equation 4.14 contains a high number of exponents, many of which when evaluated exceed the size limits for a 'double', the largest variable in Matlab. Additional operations are needed to keep the variables within the size limits in order to evaluate Equation 4.14, so even a single iteration would require high computational effort. In the end this solution was cumbersome to use and required a high degree of computational effort so an alternate solution that offers more flexibility was sought. The analytical solution was kept only as a check to test the accuracy of the numerical, which is detailed in the next section. 


\subsubsection{Numerical Solution}

For the numerical solution, the same ODE was solved, starting from an earlier point, Equation 4.9, and performing some similar substitutions as the analytical solution, $\alpha=\frac{k_{w}}{\rho_{w} C_{p, w}}$, $K=\frac{u_{w}}{\alpha_{w}}$ and $m^{2}=\frac{h P}{k_{w} A_{c}}$. The result was as follows:

$$
\frac{d^{2} T}{d x^{2}}-K \frac{d T}{d x}-m^{2}\left(T(x)-T_{\infty}\right)=0
$$

At that point central differencing was applied to the diffusion term and second order upwinding to the advection term. The result was the following:

$$
\left(\frac{T_{n+1}-2 T_{n}+T_{n-1}}{\Delta x^{2}}\right)-K\left(\frac{3 T_{n}-4 T_{n-1}+T_{n-2}}{2 \Delta x}\right)-m^{2}\left(T_{n}-T_{\infty}\right)=0
$$

The entire expression was then multipled by $\Delta x^{2}$ which lead to:

$$
T_{n+1}-2 T_{n}+T_{n-1}-\frac{K}{2 \Delta x}\left(3 T_{n}-4 T_{n-1}+T_{n-2}\right)-m^{2} \Delta x^{2}\left(T_{n}-T_{\infty}\right)=0
$$

The terms in the brackets were then expanded for future collection:

$$
T_{n+1}-2 T_{n}+T_{n-1}-\frac{3 K}{2 \Delta x} T_{n}+\frac{2 K}{\Delta x} T_{n-1}-\frac{K}{2 \Delta x} T_{n-2}-m^{2} \Delta x^{2} T_{n}+m^{2} \Delta x^{2} T_{\infty}=0
$$

The $T_{n}$ terms were then re-arranged to one side:

$$
\left(2+\frac{3 K}{2 \Delta x}+m^{2} \Delta x^{2}\right) T_{n}=T_{n+1}+\left(1+\frac{2 K}{\Delta x}\right) T_{n-1}-\frac{K}{2 \Delta x} T_{n-2}+m^{2} \Delta x^{2} T_{\infty}
$$

Finally $T_{n}$ was completely isolated to form the Finite Difference equation:

$$
T_{n}=\frac{T_{n+1}+\left(1+\frac{2 K}{\Delta x}\right) T_{n-1}-\frac{K}{2 \Delta x} T_{n-2}+m^{2} \Delta x^{2} T_{\infty}}{2+\frac{3 K}{2 \Delta x}+m^{2} \Delta x^{2}}
$$

For the purpose of the numerical solution, the bed was split into 1500 equal parts, and a steady state solution was sought with a convergence criterion of $1 \%$.

At an earlier point, a simpler numerical solution was tested, using a first order upwinding 


\subsection{Calculation of the Heat Transfer Coefficient}

scheme for the advection term, but it was abandoned in favor of the second order due to the improved accuracy of the latter with a minimal penalty in computation costs. Both numerical solutions produced a minimal difference from the analytical, as can be seen in Figure 4.2, and for this purpose the second order solution that was detailed above in Equation 4.20 was adopted for calculating the heat transfer coefficient.

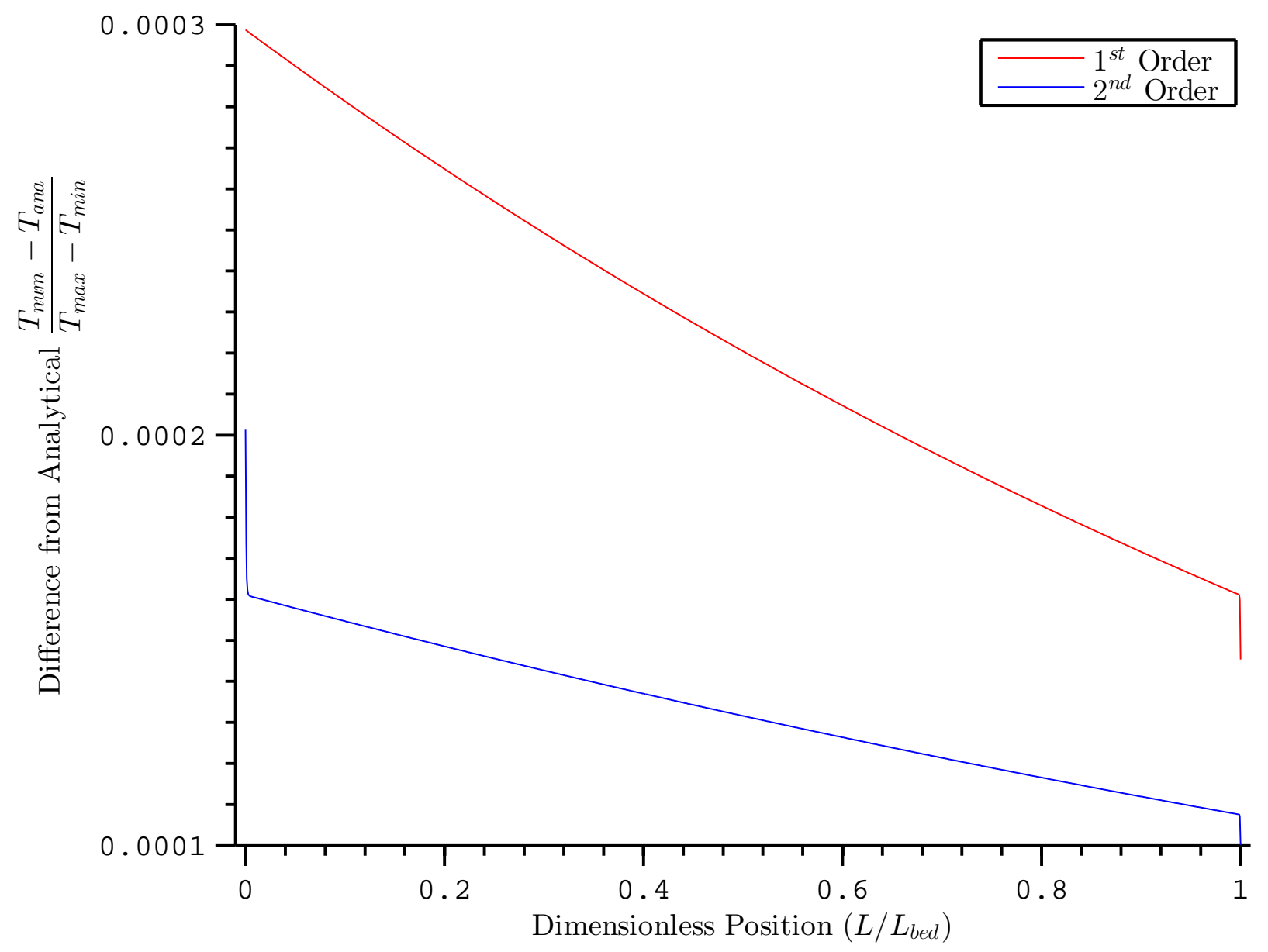

Figure 4.2: Numerical vs analytical solution 


\subsubsection{Thermometer Calibration}

The Anritsu MW-44K-TC2-ANP thermometers have a factory tolerance of $\pm 2.5^{\circ} \mathrm{C}$ at $100{ }^{\circ} \mathrm{C}$ which would have introduced an unacceptably large quantity of error into the data and limited the usefulness of the results. For this purpose they were calibrated along with the immersion thermocouple used for measuring the bed temperature, and the DT9828 DAQ. The Lauda Proline RP 1845 constant temperature baths in the Advanced Heat Transfer Laboratory were used to provide a stable environment for calibration, and the temperature in them was verified with an Omega DP97 High Accuracy Digital Thermometer accurate to $\pm 0.04^{\circ} \mathrm{C}$ and a recently certified liquid-in-glass thermometer. A fixture was constructed to hold a length of wire submerged while the various thermometers were held in place with several clamps mounted to two retort stands. The wire and contact thermometers were adjusted such that the wire just breaks the surface of the water and makes contact with the thermometer just outside the surface. A representation of the calibration setup be seen in Figure 4.3.

For the calibration procedure, all of the thermocouples were adjusted to match the correct temperature reading at $25^{\circ} \mathrm{C}$ based on the DP97 thermometer and then the temperature in the water bath was incremented by $5^{\circ} \mathrm{C}$ up to $60^{\circ} \mathrm{C}$ and back down to $25^{\circ} \mathrm{C}$, with 20 minutes given at each temperature to ensure a stable reading. The data for all of the thermocouples was recorded and plotted. This data was linear in appearance, and Matlab's Polynomial Fitting Tool was used, with a $1^{\text {st }}$ degree polynomial specified. The results of this process will be covered in the next chapter.

\section{Frictional Heating Tests}

Due to the fact that the MW-44K thermometers make physical contact with the wire and have very small thermal mass, there was a concern with frictonal heating of the thermometer resulting in artificially high readings. The inlet and outlet values would be equally influenced which would effectively cancel out, but the bed temperature is measured differently and thus 


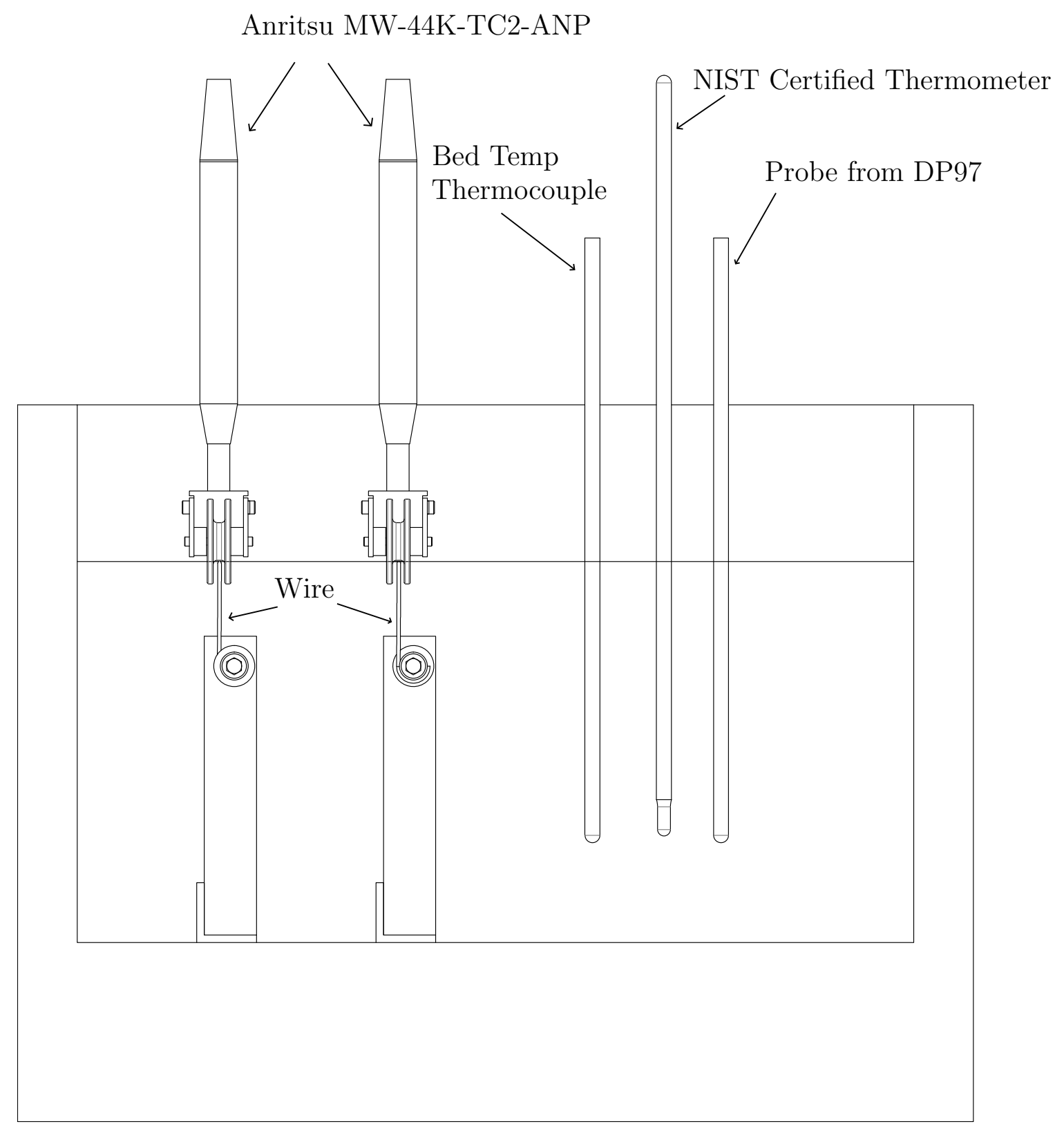

Figure 4.3: Calibration fixture and setup 
would not be subject to the same effect. For this purpose, the effect of frictional heating on the thermometers was tested in the following manner: sources of friction such as the straightener and guide pulleys were reduced as much as possible so that any frictional heating seen would be due to the thermometer heating up from friction with the wire, and the wire was run at various speeds to see how speed affects the reading. It was assumed that frictional heating, if present, could be more pronounced as wire speed increases. The results of these tests as well can be found in the next chapter.

\subsection{Data Acquisition and Processing}

Upon completing the equipment start up procedure detailed in Figure 3.8, the data acquisition was carried out by pressing start in QuickDAQ and recording for a predetermined length of time, typically between 45 and 60 seconds. While this was running, the wire speed was monitored on the Shimpo DT-105A as the unit requires that the recording button be held for the entire duration of the measurement. Upon completion of each run, the sequentially numbered file name produced by QuickDAQ, was noted in a log book, along with the maximum and minimum wire speed from that particular run. This process was repeated until all of the wire on the payoff spool was depleted, at which point the spool was rewound and left for approximately 30 minutes to cool to a steady state room temperature with the assistance of a large fan. Data acquisition was resumed after that and this was repeated until all required data was collected.

QuickDAQ stores the data in a proprietary file format that cannot be opened by other software, and requires that the data be exported from within the program to a compatible file format such as Microsoft Excel. For this purpose each data set was exported into Excel and given a file name that consists of eight digits, the first four being a two decimal representation of the maximum wire speed, and last four being the same for the minimum wire speed. For example, a data run with a maximum wire speed of $22.15 \mathrm{~m} / \mathrm{min}$ and minimum of $20.75 \mathrm{~m} / \mathrm{min}$ would become 22152075.xlsx. This file naming scheme was used for two reasons: 
to eliminate reliance on the log book, and to allow automated processing of the data by Matlab later.

\subsubsection{Data Processing}

The data processing was handled entirely by a Matlab script which makes use of custom functions to complete specific additional tasks. The script takes in the maximum and minimum wire speed, from which it decodes the file name where the data is stored and calls a custom function to open it and load all of the data into memory. It then discards the first 14 seconds of data, to ensure the wire has reached a steady state condition. Since the data contains a $45-60$ second record at $200 \mathrm{~Hz}$, some sort of division and averaging system was required to extract meaningful values from it. The chosen time period for averaging was the time it takes for the wire to traverse the bed. This way the inlet and outlet data could be compared accurately by looking at the same portion of wire. As the bed length is a known value, the script would use the wire speed to determine the time interval to be used, and divide up the rest of the record into sections of that length, discarding whatever data remains at the end that is shorter in duration than this time interval.

The script would then average all of the readings within each section to produce a value to use for calculating the heat transfer coefficient. It would take the current time interval for the inlet, the next time interval for the outlet and average the current and next time intervals for the bed temperature to most accurately reflect the conditions seen by the wire. These values would then be adjusted with the calibration factors to give the correct temperatures, and then the numerical solution from Equation 4.20 would be used iteratively to solve for the heat transfer coefficient.

To determine Nusselt number, a custom function written to evaluate the thermal conductivity of air at a given temperature was used, utilizing the correlation by Stephan and Laesecke (1985), based on the wire film temperature, which was obtained by averaging the inlet and outlet temperatures first, and then averaging that value with the bed temperature. 


\subsection{Tests Conducted}

The script would then go on to perform error analysis at each point, which will be discussed further in a later chapter. Finally, the script would collect the heat transfer coefficient values over all of the intervals, associated error, Nusselt numbers and their associated errors and save all of it to a Matlab data file named after the wire speed in the same manner as before. The entire script as well as the functions it calls can be found in the Appendix.

\subsection{Tests Conducted}

\subsection{1 $\mathrm{U}_{m f}$ Comparisons}

Previous work by Friedman et al. (2006) showed that heat transfer to small, wire sized cylinders does not change appreciably after $2.5 \times U_{m f}$, which would imply that testing both different wire speeds and fluidizing velocities would be unnecessary, and that a single fluidizing velocity could be used for all tests. To ensure that these findings still hold true for moving wire, wire speeds of 20 and $40 \mathrm{~m} / \mathrm{min}$ were tested at 2,3 and $4 \times U_{m f}$. The details can be seen in Table 4.1. These tests were only carried out with the 60 grit sand as there was no reason to believe that the results would be any different with the smaller sand particles.

Table 4.1: Tests at various values of $U_{m f}$ and Wire Speed

\begin{tabular}{lcl}
\hline $2 \times U_{m f}$ & $3 \times U_{m f}$ & $4 \times U_{m f}$ \\
\hline $20 \mathrm{~m} / \mathrm{min}$ & $20 \mathrm{~m} / \mathrm{min}$ & $20 \mathrm{~m} / \mathrm{min}$ \\
$40 \mathrm{~m} / \mathrm{min}$ & $40 \mathrm{~m} / \mathrm{min}$ & $40 \mathrm{~m} / \mathrm{min}$ \\
\hline
\end{tabular}

\subsubsection{Experimental Matrix}

All of the tests meant to determine the effect of wire speed on heat transfer were carried out at $3 \times U_{m f}$. The wire speed was varied in increments of $10 \mathrm{~m} / \mathrm{min}$ from $10-50 \mathrm{~m} / \mathrm{min}$ and then to $70 \mathrm{~m} / \mathrm{min}$ in increments of $5 \mathrm{~m} / \mathrm{min}$. A full list of the tests can be seen in Table 4.2 . 
Table 4.2: Test Matrix. All tests conducted at a Fluidizing rate of $3 \times U_{m f}$

\begin{tabular}{ccccccccccc}
\hline \multirow{2}{*}{$\begin{array}{c}\text { Sand Grit } \\
60\end{array}$} & Mean Particle Size & \multicolumn{8}{c}{ Wire Speed m/min } \\
\cline { 3 - 10 } & $250 \mu m$ & 10 & 20 & 30 & 40 & 50 & 55 & 60 & 65 & 70 \\
70 and 80 Mix & $204 \mu m$ & 10 & 20 & 30 & 40 & 50 & 55 & 60 & 65 & 70 \\
\hline
\end{tabular}




\section{CHAPTER 5}

\section{Results and Discussion}

\subsection{Properties of $\mathrm{Al}_{2} \mathrm{O}_{3}$ Particles}

$U_{m f}$ is a crucial parameter in fluidization (Equations 2.4, 2.9), as it is key to determining fluidized bed behavior and appears in some form in nearly all correlations. For these reasons it is important that it is known with a fair degree of certainty. It relies heavily upon particle properties, so the particles needed to be analyzed and classified according to their Geldart Groups (Geldart, 1973). The 8 and 60 grit particles were analyzed by Koundakjian (2002), and the results of that study are presented in Table 5.1.

The mixture of 70 and 80 grit particles were newly introduced in this work, and their properties were determined in the following manner: the mean particle diameter was determined

Table 5.1: Properties of 8 and 60 grit particles

\begin{tabular}{lll}
\hline Properties & $\# 8 \mathrm{Al}_{2} \mathrm{O}_{3}$ Sand & $\# 60 \mathrm{Al}_{2} \mathrm{O}_{3}$ Sand \\
\hline Mean Particle Diameter, $d_{p}$ & $2210 \mu m$ & $254 \mu \mathrm{m}$ \\
Particle Density, $\rho_{s}$ & $3970 \frac{\mathrm{kg}}{\mathrm{m}^{3}}$ & $3970 \frac{\mathrm{kg}}{\mathrm{m}^{3}}$ \\
Geldart's Classification & Group D & Group B \\
Fluidized Behavior & No Fluidization & Bubbling occurs at or only \\
& & slightly above $U_{m f}$ Good \\
Bulk Density of bed, $\rho_{b}$ & - & solids mixing. \\
Sphericity, $\phi_{s}$ & - & $2124 \frac{\mathrm{kg}}{\mathrm{m}^{3}}$ \\
Average Packed Bed Voidage, $\varepsilon_{a v g}$ & - & 0.66 \\
Minimum Bed Voidage at $U_{m f}, \varepsilon_{m}$ & - & 0.47 \\
\hline
\end{tabular}


using sieve analysis, the sphericity was taken from the literature as the commonly accepted value for sharp sand (Holdich, 2002), the particle density was taken as the reference value for $\mathrm{Al}_{2} \mathrm{O}_{3}$, the average packed bed voidage was determined using an experimental technique found in Koundakjian (2002), and the minimum bed voidage at $U_{m f}$ was determined by using the sphericity and Equation 2.11.

The Sieve Analysis process is performed by stacking a set of sieves, starting from finest at the bottom, to coarsest at the top. The sample of particles is then poured into the top sieve and the lid is fastened securely. The entire stack of sieves is then placed into a sieve shaker, which is turned on and left to run for approximately 10 minutes. The sieve shaker performs both shaking and tapping actions vigorously, ensuring that the particles have all filtered down to the respective sieve size. The particles are then removed from each sieve and weighed to get an overall size distribution. Sieve Analysis was carried out on both sets of particles. In the case of the $70 \& 80$ grit mix, it was necessary to determine the size, and in the case of the 60 grit particles, it served to verify Koundakjian (2002)'s findings. The results of these tests can be seen in Figures 5.1 and 5.2.

Koundakjian's experimental technique for finding the voidage of a packed bed requires that the weight and minimum packed volume of a sample of particles be determined first. An OHAUS Precision Standard TS200S digital scale was used to find the weight of a beaker filled with the sample of particles, as well as the empty beaker weight itself. From this, the weight of the particles was obtained. The volume was then determined by connecting that same beaker to the housing of a large blower in the laboratory, and leaving it to run for an hour so that the vibrations produced by the blower can be transmitted into the beaker to agitate the particles, causing them to re-arrange themselves into their most densely packed configuration. The volume was then read off of the beaker, which is accurate to $\pm 1 \mathrm{ml}$, and the bulk density was calculated. The bulk density and particle density were then used in the definition of voidage, $\varepsilon=1-\frac{\rho_{b}}{\rho_{s}}$ to determine it within \pm 0.0066 .

The findings from these experiments are outlined in Table 5.2. 


\subsection{Properties of $\mathrm{Al}_{2} \mathrm{O}_{3}$ Particles}

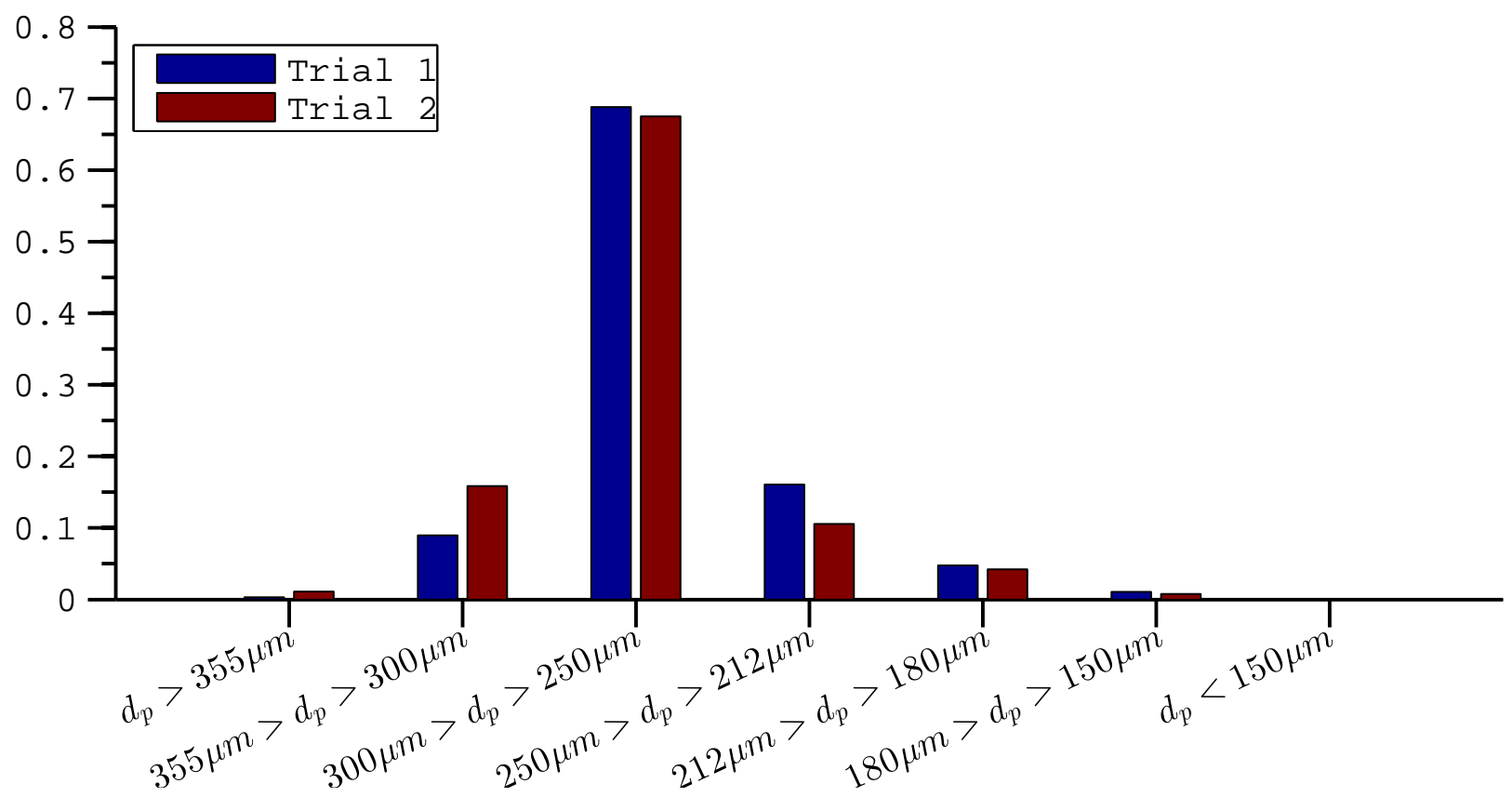

Figure 5.1: Size distribution of 60 grit particles

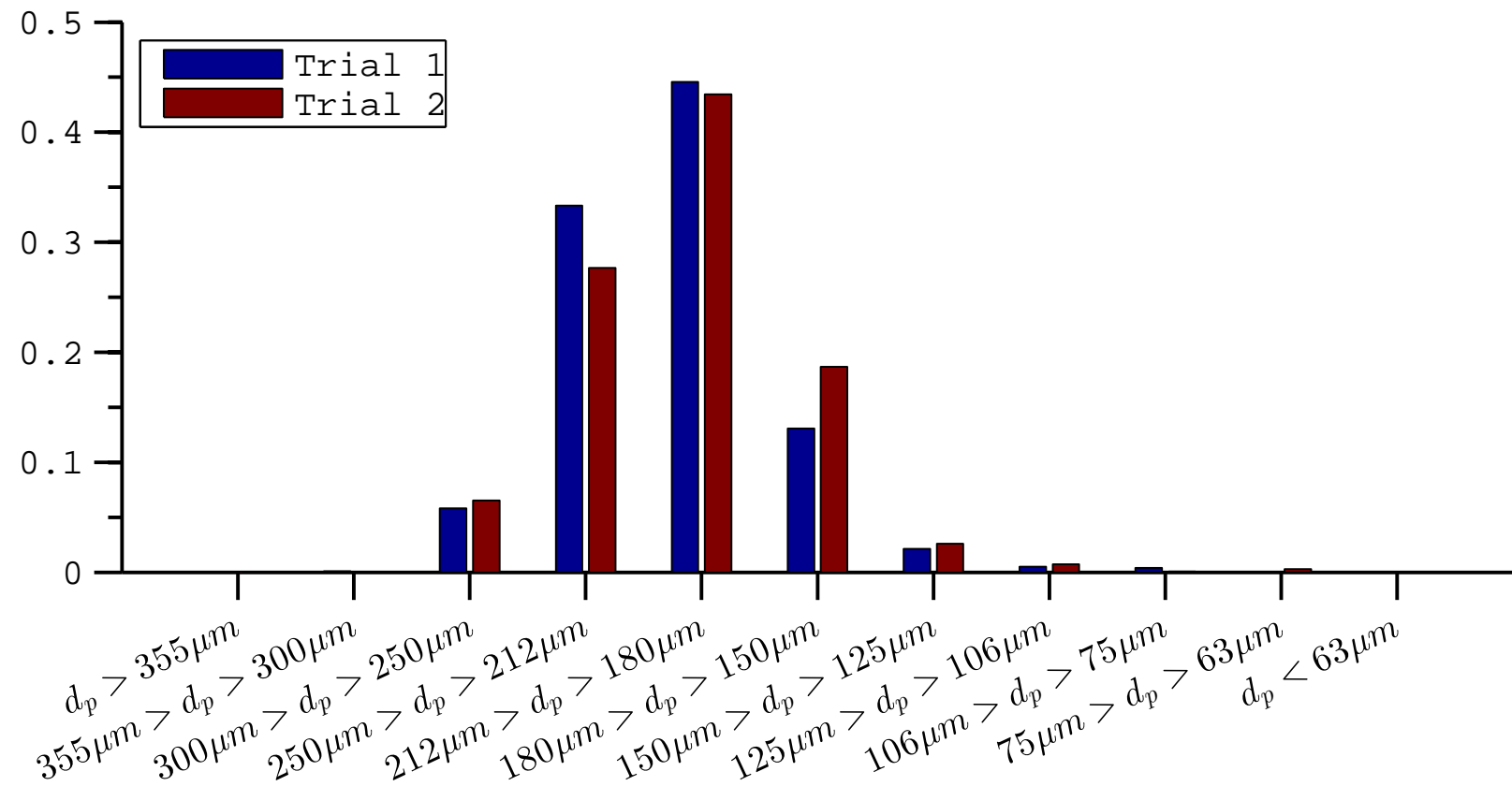

Figure 5.2: Size distribution of $70 \& 80$ grit particle mix 


\subsection{Thermometer Calibration and Testing}

Table 5.2: Properties of $70 \& 80$ grit mix particles

\begin{tabular}{ll}
\hline Properties & $\# 70 \& 80$ Grit Mix $\mathrm{Al}_{2} \mathrm{O}_{3}$ Sand \\
\hline Mean Particle Diameter, $d_{p}$ & $204 \mu \mathrm{m}$ \\
Particle Density, $\rho_{s}$ & $3970 \frac{\mathrm{kg}}{\mathrm{m}^{3}}$ \\
Geldart's Classification & Group B \\
Fluidized Behavior & Bubbling occurs at or only slightly \\
& above $U_{m f}$. Good solids mixing. \\
Bulk Density of bed, $\rho_{b}$ & $2200 \frac{\mathrm{kg}}{\mathrm{m}^{3}}$ \\
Sphericity, $\phi_{s}$ & 0.66 \\
Average Packed Bed Voidage, $\varepsilon_{a v g}$ & 0.45 \\
Minimum Bed Voidage at $U_{m f}, \varepsilon_{m f}$ & 0.54 \\
\hline
\end{tabular}

\subsection{Thermometer Calibration and Testing}

\subsubsection{Calibration}

All of the thermometers were calibrated along with the DAQ according to the procedure presented in the previous chapter. This involved inserting them into a constant temperature bath, and cycling up and down through the temperature range that would be encountered in the experiments. The thermometers and DAQ were "zeroed" at $25^{\circ} \mathrm{C}$, prior to running the temperature up and down, to make the correction process simpler and more reliable. After all of the temperatures were tested, it was observed that the discrepancy between the value reported by the Anritsu contact thermometers and the actual value increased with the bath temperature, however, this was not the case for the immersion thermocouple. It was necessary at this point to determine if the Anritsu thermometers are under-reporting, or if the wire surface that they are contacting is cooler than the bath temperature. For this purpose, a model was constructed in FEHT to simulate the calibration process and ascertain whether the wire is at a uniform temperature as previously assumed. As FEHT is not capable of handling combined fluid flow and heat transfer problems, the domain had to 


\subsection{Thermometer Calibration and Testing}

be restricted to the wire and water bath, and a convection boundary condition was applied on the upper surface of the wire and the water to simulate the natural convection from the air. To determine the natural convection heat transfer coefficient, a correlation for natural convection from a horizontal flat plate in air was used (Simons, 2001):

$$
h=C\left(\frac{\Delta T}{L}\right)^{n}
$$

$$
\text { with } \begin{aligned}
C & =0.59 \text { and } \\
n & =0.25 \text { for a horizontal plate } \\
\Delta T & =\text { Difference between surface and air temperatures } \\
L & =\text { Length of surface }
\end{aligned}
$$

The worst case scenario was analyzed, with the surface temperature taken as $60^{\circ} \mathrm{C}$ and the

air $24^{\circ} \mathrm{C}$, which produced a convection coefficient due to natural convection of $2.57 \frac{\mathrm{W}}{\mathrm{m}^{2} \mathrm{~K}}$. This value for convection coefficient was used in FEHT along with an air temperature of $24^{\circ} \mathrm{C}$. In reality, there is significant heating of the air near the water surface, and the local air temperature would be much higher than $24^{\circ} \mathrm{C}$, however, the exact temperature is difficult to obtain, and as such the extreme case of it being the same as ambient air was analyzed.

To ensure constant temperature inside the bath for this simulation, nodes were selected at random and set to the bath temperature. The walls of the bath and the lower immersed portion of the wire were also set to the bath temperature. This resulted in a uniform temperature for all of the water in the bath, which is an accurate representation of reality, as the Lauda Proline RP 1845 contant temperature bath circulates the water constantly to ensure uniformity.

The simulation predicted a temperature of $59.85^{\circ} \mathrm{C}$ at the center on the upper wire surface which is exposed to air and makes contact with the thermometer. Since this result was obtained assuming an unrealistically high temperature gradient at the surface, in practice the heat loss to the air would be far lower, and the upper surface would almost certainly be 


\subsection{Thermometer Calibration and Testing}

at the same temperature as the bath and the rest of the wire, therefore any discrepancies are due to the thermometers, and not the physics of the system. Figure 5.3 is a visual representation of the simulation, as well as the temperature gradients that were observed.

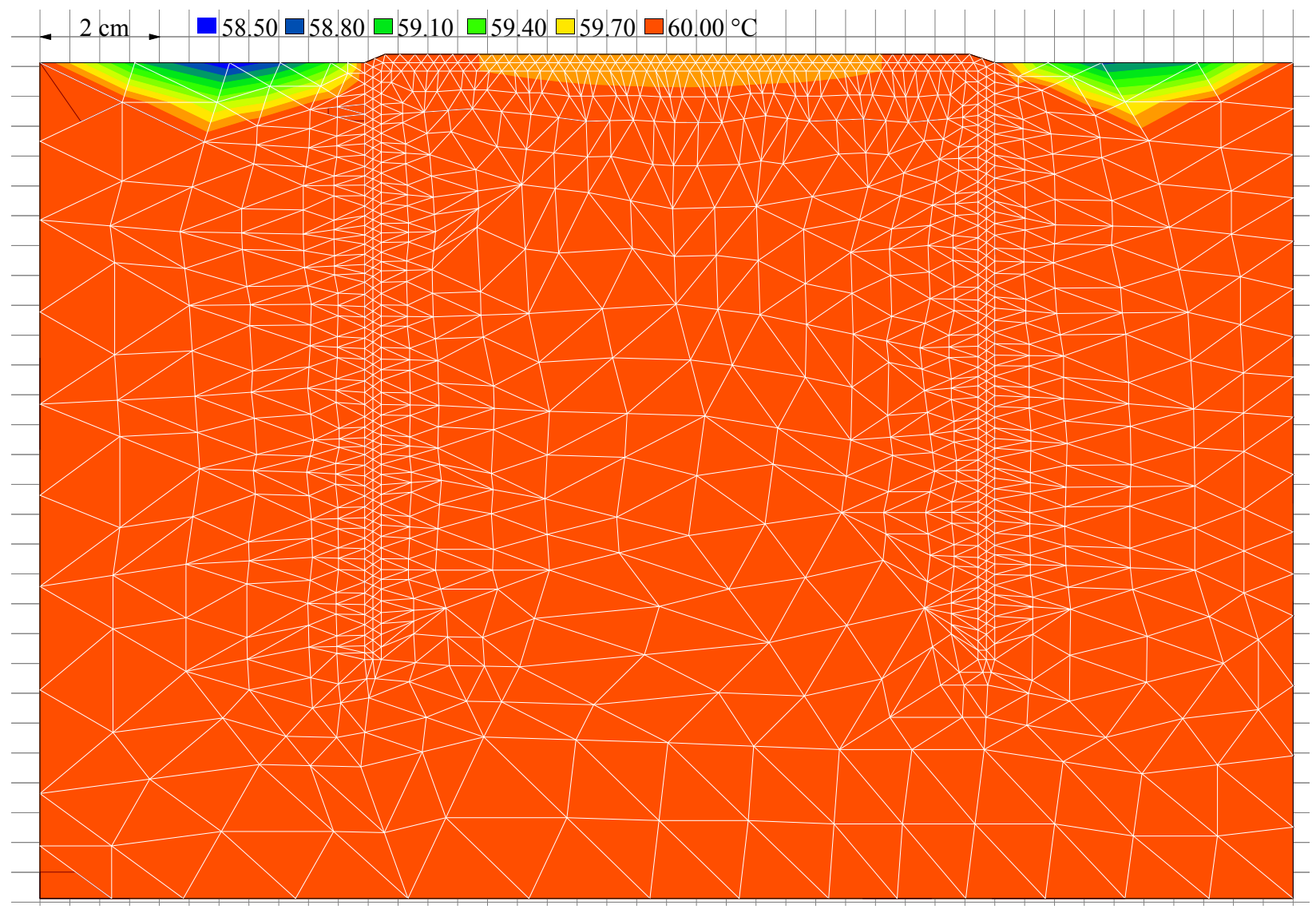

Figure 5.3: FEHT model of calibration apparatus

After confirming the wire temperature, the measured values by the thermometer were plotted against the reference values from the Omega DP97 high accuracy thermometer that was monitoring the constant temperature bath. The following equations for correction were 
determined using Matlab's Polynomial Fitting Tool:

$$
\begin{aligned}
T_{\text {bed }_{\text {real }}} & =T_{\text {bed }_{\text {measured }}} \times 0.9906+0.2586 \\
T_{\text {in }_{\text {real }}} & =T_{\text {in }_{\text {measured }}} \times 1.0197-0.422 \\
T_{\text {out }_{\text {real }}} & =T_{\text {out }} \text { measured } \\
\quad \text { for } & 25^{\circ} \mathrm{C}<\mathrm{T}<60^{\circ} \mathrm{C}
\end{aligned}
$$

Figure 5.4 shows a comparison between the temperature values that would be obtained by using the equations, and the actual measured values from the calibration. It can be seen that the equations can predict the temperature within a range of $\pm 0.1^{\circ} \mathrm{C}$ therefore this value was used for their tolerance in the error analysis.

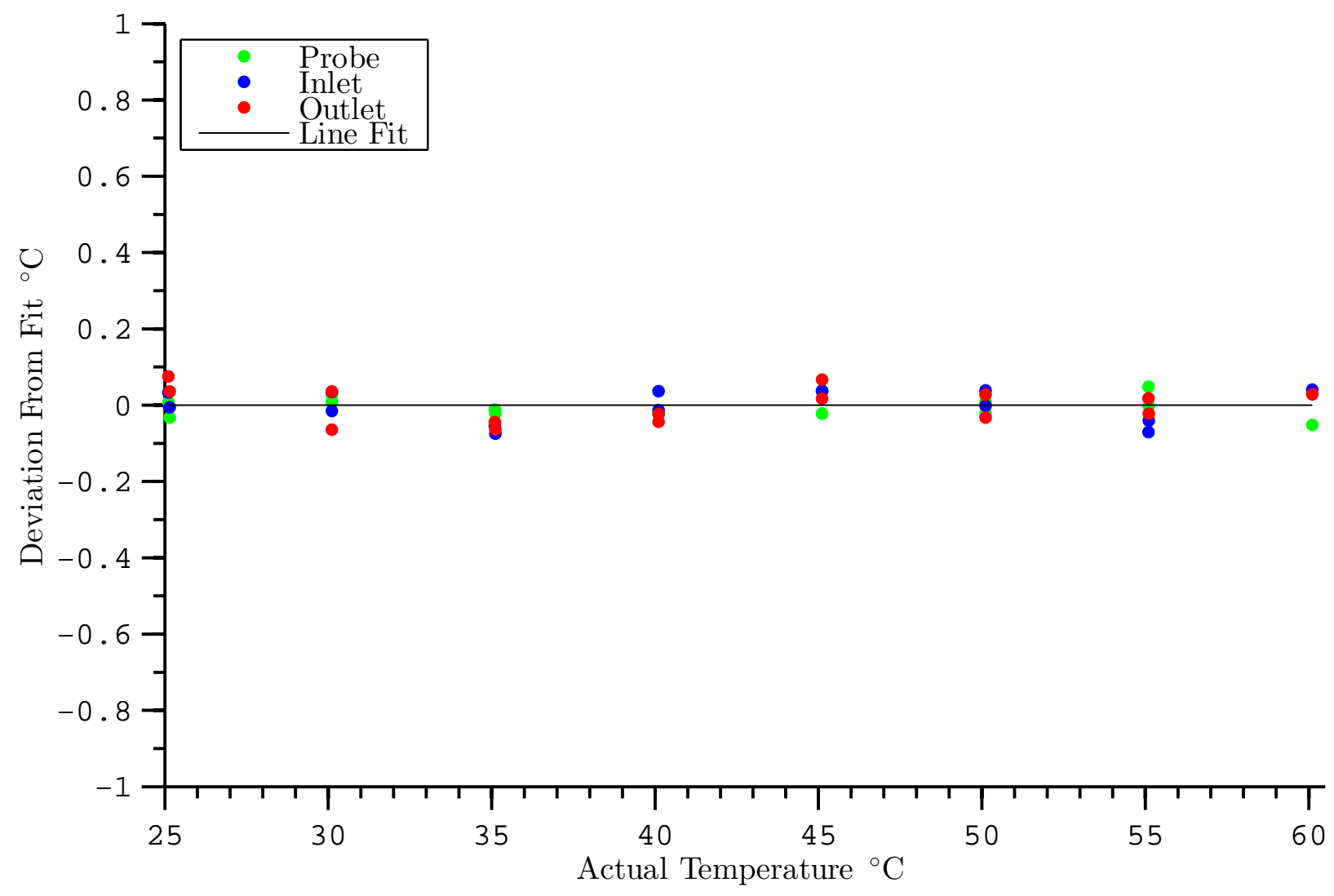

Figure 5.4: Calibration data 


\subsubsection{Frictional Heating}

If any frictional heating of the Anritsu contact thermometers was present it would contribute an additional source of error that can alter the calculated value for the heat transfer coefficient. This was tested by minimizing external sources of friction to observe what effect if any wire speed would have on the readings. The apparatus was ran at different wire speeds and the temperature at the inlet was recorded. The results can be seen in Figure 5.5. The temperature variation between the speeds is very minor, and easily attributable to external sources of friction which could not be fully eliminated.

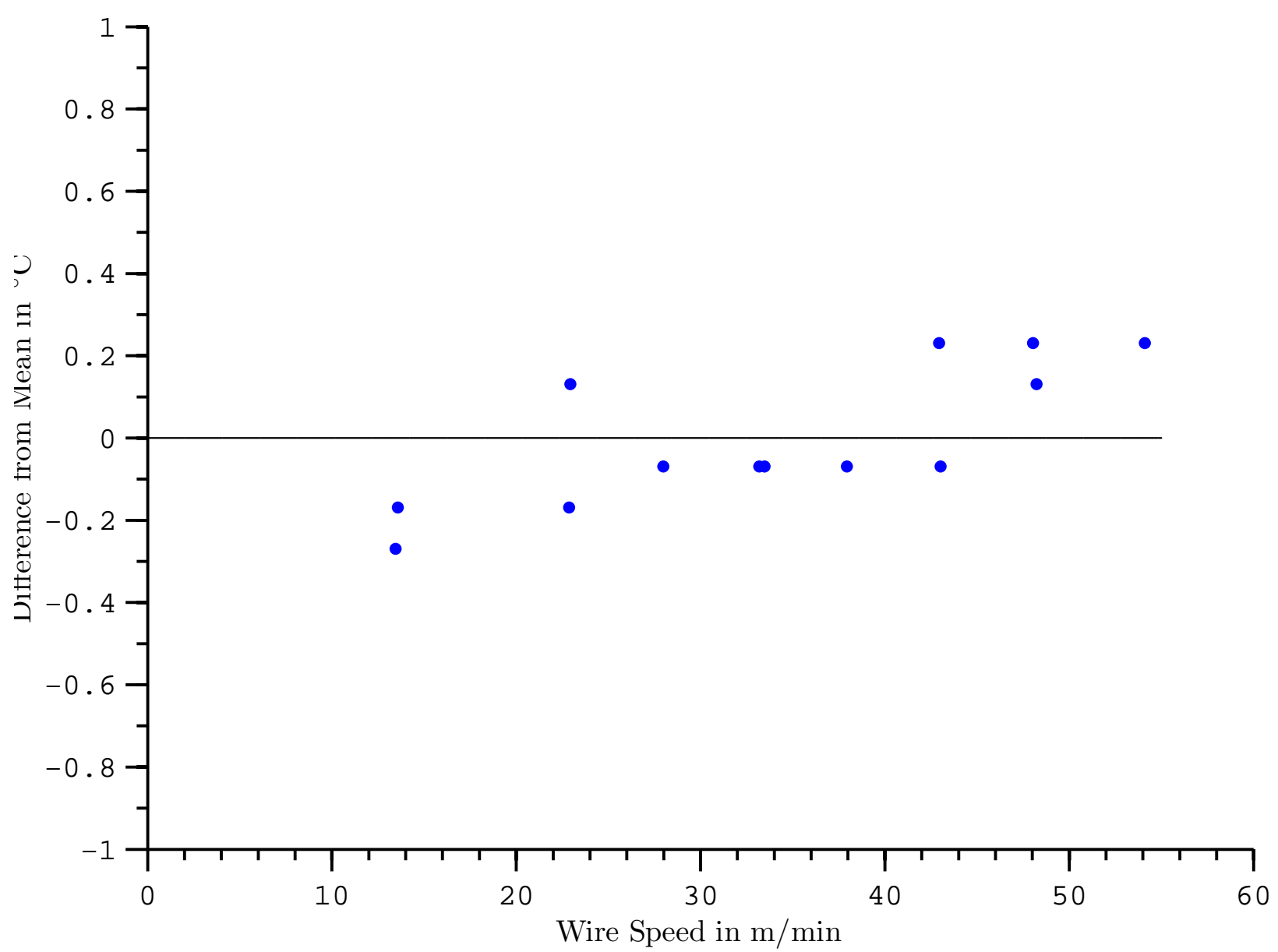

Figure 5.5: Variations in inlet temperature vs wire speed 


\section{$5.3 U_{m f}$ Tests}

Previous work by Friedman et al. (2006) has shown that for immersed small cylinders that are kept static, there is no dependence of Nusselt number on fluidizing velocity past $2.5 \times U_{m f}$. There was no information available on whether this trend holds true if they are moving, so tests were conducted to investigate the relationship between fluidizing velocity and heat transfer. Three fluidizing velocities were tested, $2 \times U_{m f}$, chosen to be under Friedman et al.'s threshold, and $3 \& 4 \times U_{m f}$, chosen to be above. The wire used in this and all further tests was $2.8 \mathrm{~mm}$ Aluminum $1188 \mathrm{H} 18$ wire as previously mentioned in the Experimental Procedure chapter. Two wire velocities were tested at each fluidizing velocity, $20 \& 40 \mathrm{~m} / \mathrm{min}$ for a total of 6 tests, the results of which can be seen in Figure 5.6.

As can be seen from Figure 5.6, there is a pronounced difference in Nusselt number (13\% on average) between 2 and $3 \times U_{m f}$, but the difference between 3 and $4 \times U_{m f}$, is far smaller ( $5 \%$ on average), and their respective error bars overlap. This is consistent with Friedman et al.'s findings for static immersed small cylinders and as such eliminates fluidizing velocity as a parameter, and removes the need for further tests to be carried out at various fluidizing velocities. As a result of these findings, all subsequent tests were carried out at approximately $3 \times U_{m f}$. Industrial fluidized bed furnaces typically operate at fluidizing velocities between 2.5 and $5 \times U_{m f}$, making it a suitable choice.

\subsection{Grit Tests}

The first round of tests were performed with 60 grit sand to ensure comparability with earlier works by Friedman et al. as well as Koundakjian. The tests were conducted at a fluidizing velocity of $2.85 \times U_{m f}$, with wire speed being varied from $10 \mathrm{~m} / \mathrm{min}$ to slightly under 75 $\mathrm{m} / \mathrm{min}$. The results can be seen in Figure 5.7. Because the data processing method splits each run into intervals matching the residence time of the wire inside the bed, each point on this plot represents a snapshot of the conditions that the specific moving element of 


\subsection{Grit Tests}

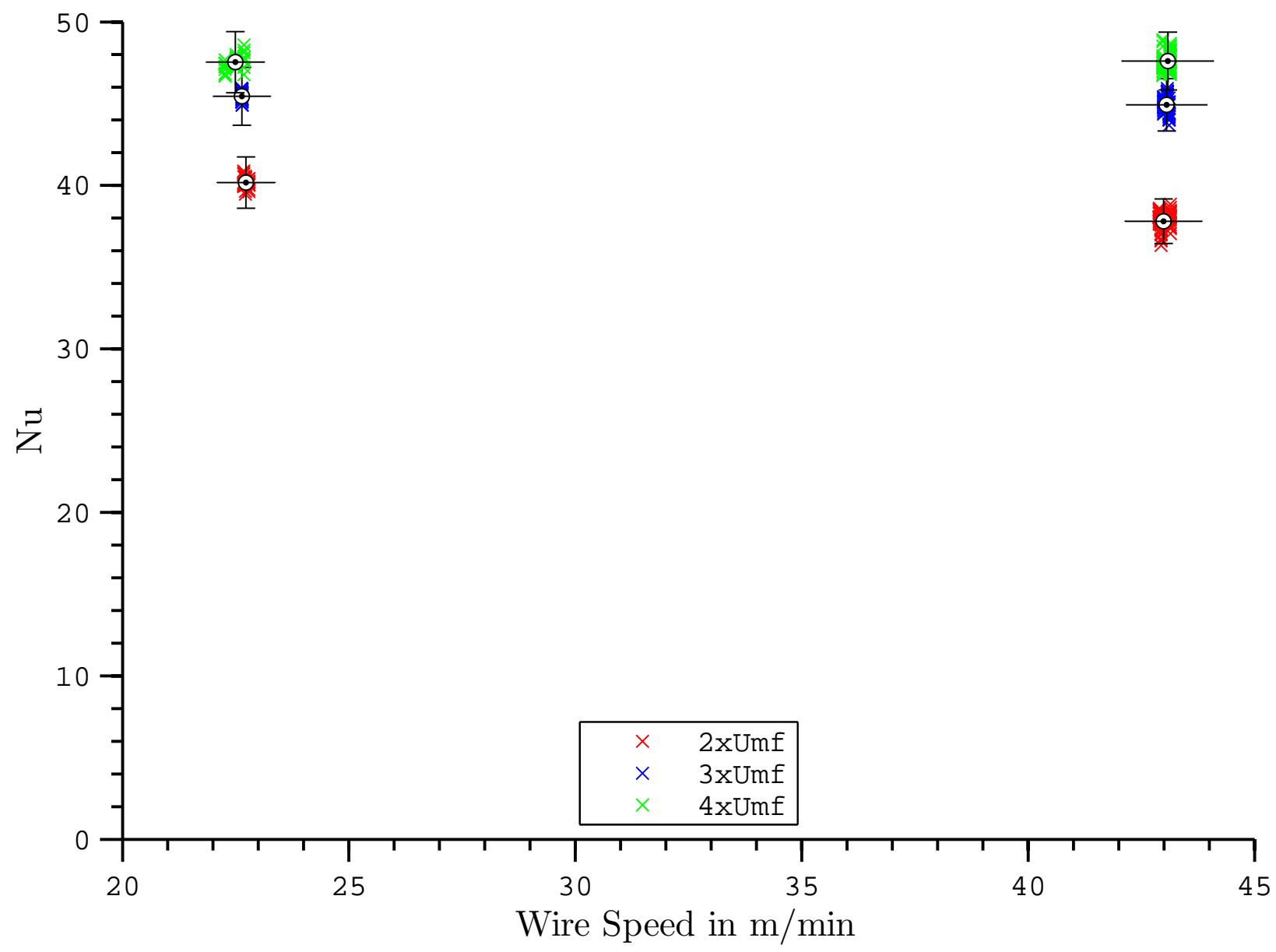

Figure 5.6: Nusselt numbers at various fluidizing velocities and wire speeds

wire experienced. The error bars are located at the average value for each cluster of points. The horizontal error bars indicate the range of wire speed that those points can lie on, based on the readings collected from the digital tachometer. The vertical error bars on the other hand, are calculated based on the combined experimental error from all measurement devices, including the thermometers, DAQ, tachometer, as well as the tolerances on the wire diameter and bed length. The formulation used for the error analysis which produced the vertical error bars will be discussed in a later chapter on error analysis. 


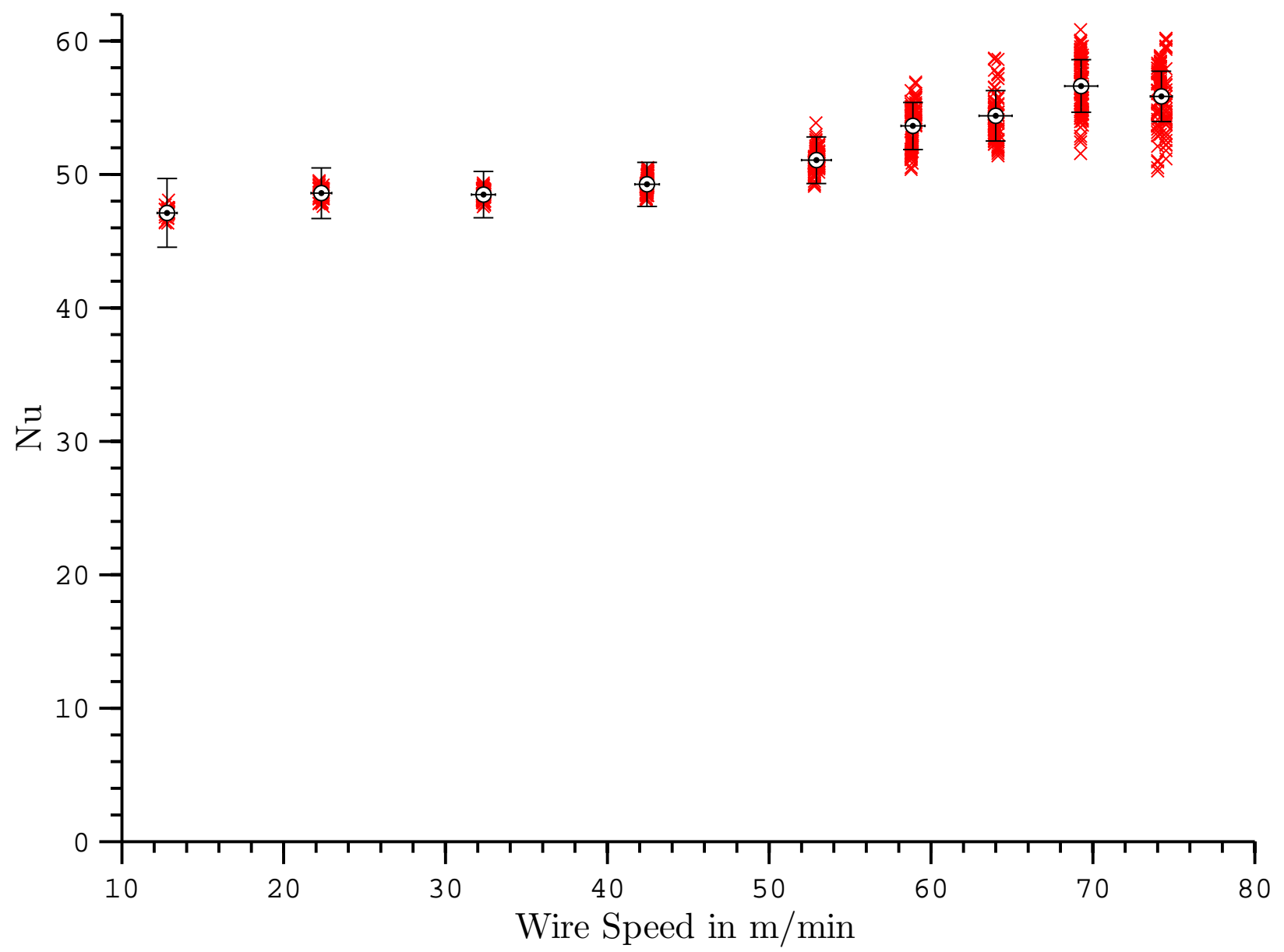

Figure 5.7: Nusselt numbers at various wire speeds in 60 grit sand

\section{$5.5 \quad 70-80$ Grit Mix Tests}

A second round of tests was performed using a mixture of 70 and 80 grit sand particles. All previous studies of heat transfer to immersed surfaces have shown a dependence on particle size, but it is unclear whether variations in wire speed would produce different results for different particle sizes. These tests were performed at a fluidizing velocity of $3.5 \times U_{m f}$ with the same wire that was used in the previous tests. The range of wire speeds tested was 10-75 $\mathrm{m} / \mathrm{min}$ as in the previous set. The results can be seen in Figure 5.8. Once again, each point is a snapshot of the conditions experienced by a moving element of wire, and the error bars 


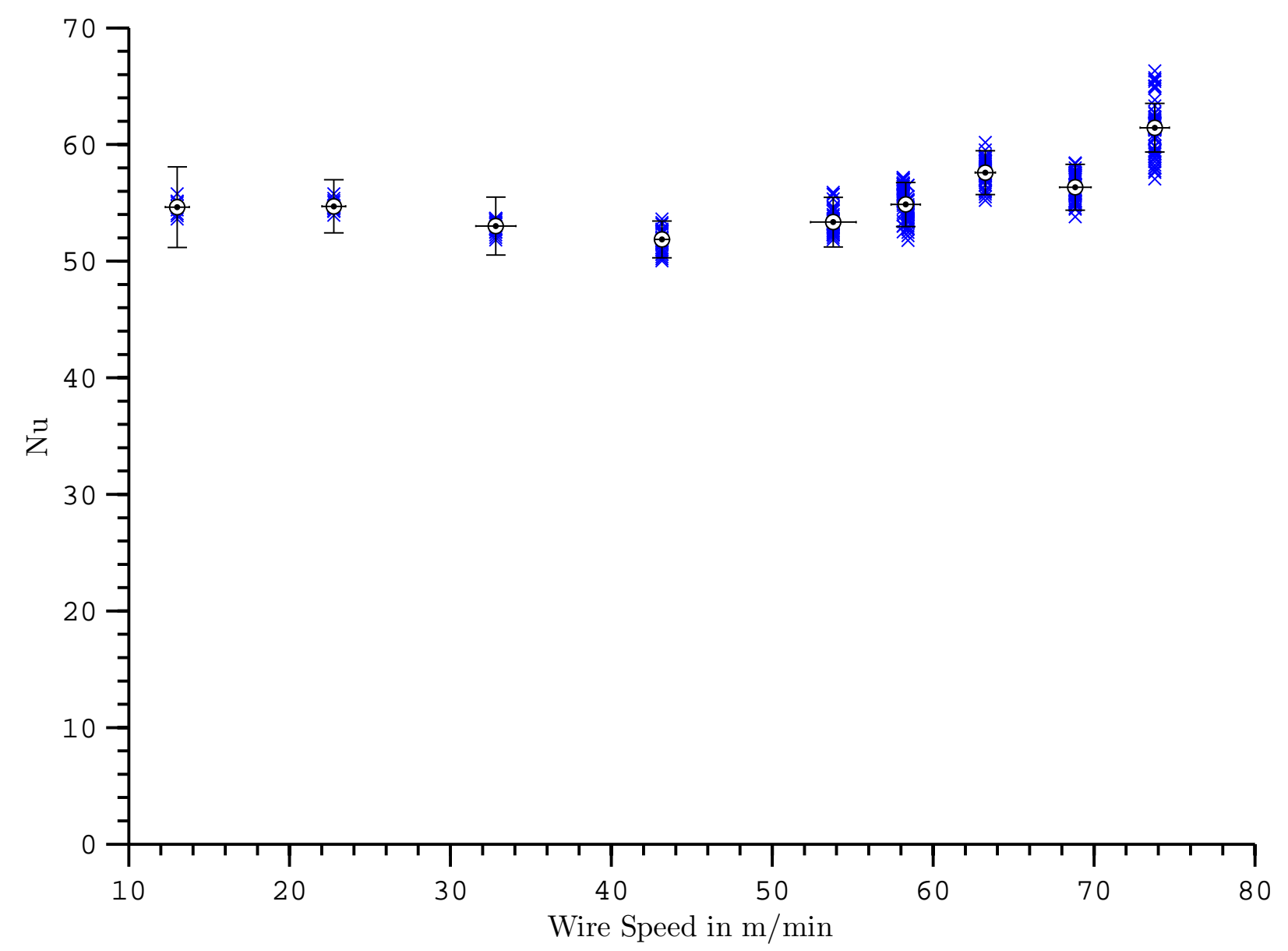

Figure 5.8: Nusselt numbers at various wire speeds in a mix of 70 and 80 grit sand

are determined and positioned according to the same principles as the ones in the previous set of data.

\subsection{Discussion of Results}

Figures $5.7 \& 5.8$ illustrate that wire speed does play a role in the heat transfer to a moving wire immersed in a fluidized bed furnace. While the behavior exhibited in beds of different particles is not identical, there is an overall trend that shows Nusselt numbers increasing with 


\subsection{Discussion of Results}

wire speeds. Through comparison of the different data sets and with correlations obtained from literature, additional insight into this behavior was gleaned, which is presented in the following sections.

\subsubsection{Comparison of 60 and 70-80 Grit Mix}

To gain some insight into what effect the different particle sizes combined with varied wire speed have on heat transfer, the two data sets were plotted together, and can be seen in Figure 5.9. The scatter from the previous plots was replaced with single points that represent the average values, and error bars that now represent statistical scatter, as opposed to experimental error as in the previous plots.

The data set for the finer particles in the $70 \& 80$ Grit mix shows higher Nusselt numbers, as expected from previous works, however, the spacing between the two data sets is not at all equal. The largest gap can be seen at lower wire speeds, around 10-30 $\mathrm{m} / \mathrm{min}$, with the gap drastically narrowing between $40-65 \mathrm{~m} / \mathrm{min}$, only to widen again at $74 \mathrm{~m} / \mathrm{min}$. As the comparison of the two data sets raised as many questions as it answered, additional analysis was performed to gain insight into the different behaviors in the two data sets.

\section{Statistical Scatter}

Before continuing onto further comparisons, an important note on statistical scatter and the role it plays needs to be addressed. As previously mentioned, each data point is based on a time interval equal to the residence time of a single element of wire inside the fluidized bed. Since the wire speed is varied, the length of these time intervals is varied as well. At the lowest speed, this time interval is as long as $5.3 \mathrm{~s}$, while at the highest it is less than $0.8 \mathrm{~s}$. Gas-fluidized beds of Group B particles such as the one used in these experiments exhibit a large degree of bubbling, which is a highly stochastic process. A study by Boerefijn et al. (1999) looking at the instantaneous heat transfer coefficient to an immersed hot wire probe, 


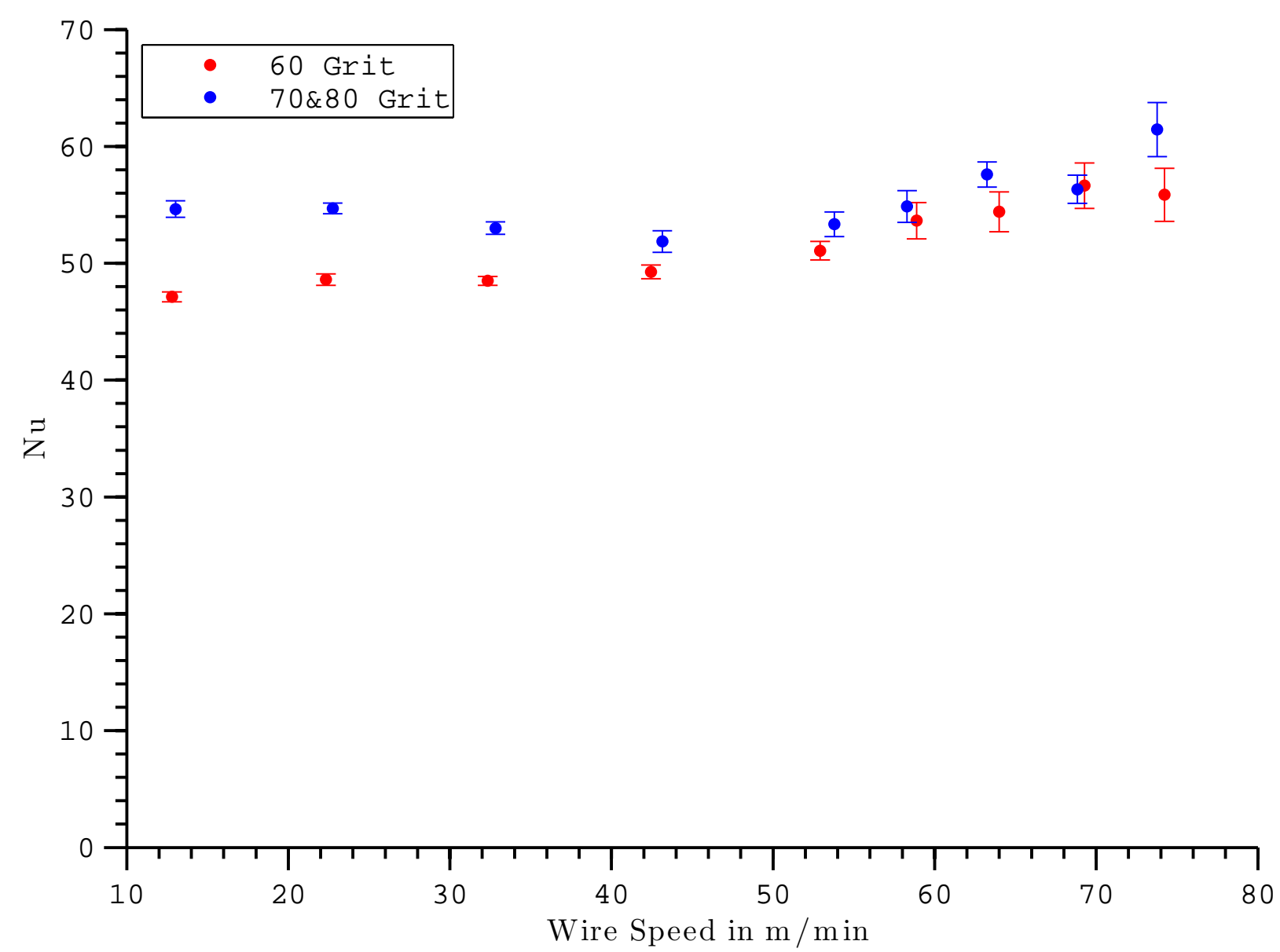

Figure 5.9: Nusselt numbers at various wire speeds in two different sets of particles

showed variations as high as $4-500 \%$. The same study, however, also showed that while instantaneous rates were chaotic, when time averaged over a sufficiently long time interval, the values for heat transfer coefficient were quite consistent.

This same trend can be observed in both data sets in this study, as the statistical scatter is much higher at the higher wire speeds, when the wire spends far less time inside the fluidized bed. Another important note is the difference in lengths between the fluidized bed in this study and those used in industry, especially since the purpose of this study is to facilitate industry use of fluidized beds. The typical industrial fluidized bed is approximately $20 \mathrm{~m}$ in length, as opposed to the $0.965 \mathrm{~m}$ of the unit used for these experiments. The difference in 


\subsection{Discussion of Results}

length changes the time interval that a wire element would spend in the bed at the highest speed, from less than $0.8 \mathrm{~s}$ to $16.2 \mathrm{~s}$, and from $5.3 \mathrm{~s}$ to $109 \mathrm{~s}$ at the lowest speed. The implication of this being that in actual industry use, the statistical scatter observed in this study would disappear almost completely due to the long residence times of the wire inside the bed.

\section{h vs $\mathrm{Nu}$}

The Nusselt number is the ratio of convective to conductive heat transfer, and is defined as:

$$
N u=\frac{h D_{t}}{k_{g}}
$$

The wire diameter, $D_{t}$, is not varied in this study, but $k_{g}$, the thermal conductivity of the gas, which is air in this case, does vary significantly with temperature. Due to the type of thermostat employed for the heater and the high thermal mass of the fluidized bed, there were wide variances present in temperature. Since the film temperature around the wire varies quite a bit, Nusselt number was plotted along with the heat transfer coefficient, h, for both data sets to see the influence of film temperature.

Figure 5.10 shows h and Nusselt number for the 60 grit sand. The shapes of both curves are nearly identical as the temperatures were better controlled for this data set.

For the $70 \& 80$ grit mix, Figure 5.11, there is a significant difference between $\mathrm{h}$ and $\mathrm{Nu}$ at lower wire speeds, and it nearly disappears at higher ones. This is due to the fact that there were larger variations in film temperature in the second data set. These findings illustrate the importance of using Nusselt number as a comparison instead of the heat transfer coefficient, $\mathrm{h}$, as $\mathrm{h}$ can be inflated at higher temperatures, due to the increased thermal conductivity of the gas, leading to a misinterpretation of results. It also highlights that care should be taken when defining the film temperature, as it can influence the results. In this case it was defined as:

$$
T_{\text {film }}=\frac{\left(\frac{T_{\text {in }}+T_{\text {out }}}{2}\right)+T_{\text {bed }}}{2}
$$




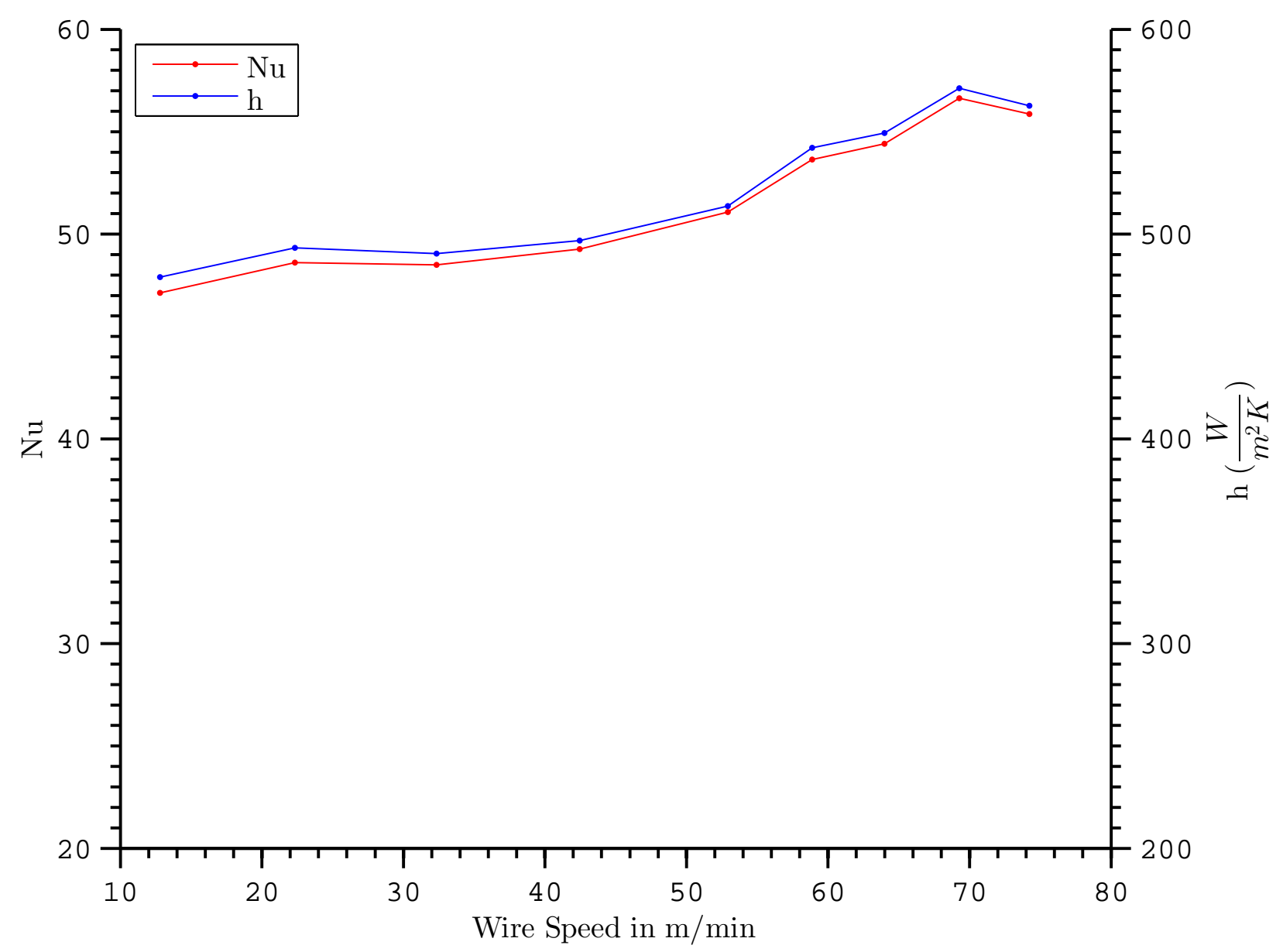

Figure 5.10: $\mathrm{h}$ and Nusselt number for 60 grit sand

\subsubsection{Comparison to Existing Correlations}

To better understand the data and aid in the development of a correlation to predict the Nusselt number, the wire speed was non-dimensionalized by dividing it by $U_{m f}$, and it was re-plotted along with existing correlations by Grewal and Saxena (1980), Friedman et al. (2006) and Masoumifard et al. (2008). Figure 5.12 is the plot in question.

The non-dimensionalization of the $\mathrm{x}$-axis combined with the different axis scaling shows greater similarity between the two data sets, while the plot overall confirms previous findings by Friedman et al. and Masoumifard et al. about the applicability of correlations developed 


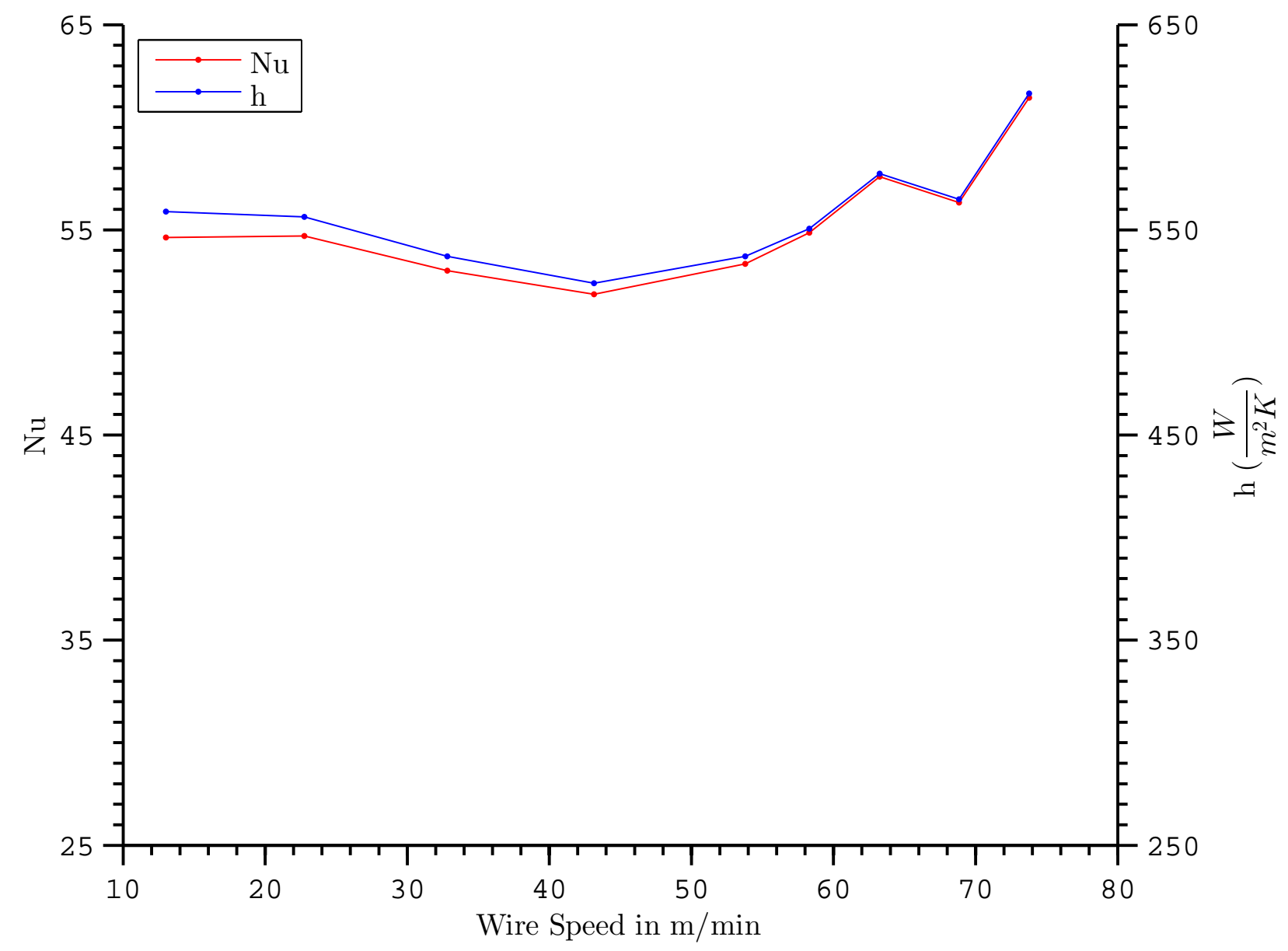

Figure 5.11: h and Nusselt number for a mix of 70 and 80 grit sand

for larger cylinders. The Grewal and Saxena (1980) correlation, which was developed for medium and large sized cylinders, grossly over-predicts the heat transfer for both data sets, while both Friedman et al.'s and Masoumifard et al.'s correlations are quite close to where a zero wire velocity point would be expected based on extrapolation of the data obtained.

As the wire speed increases, however, there is a departure from the existing correlations, which highlights a need for a new correlation that accounts for wire movement to be developed. 


\subsection{Discussion of Results}

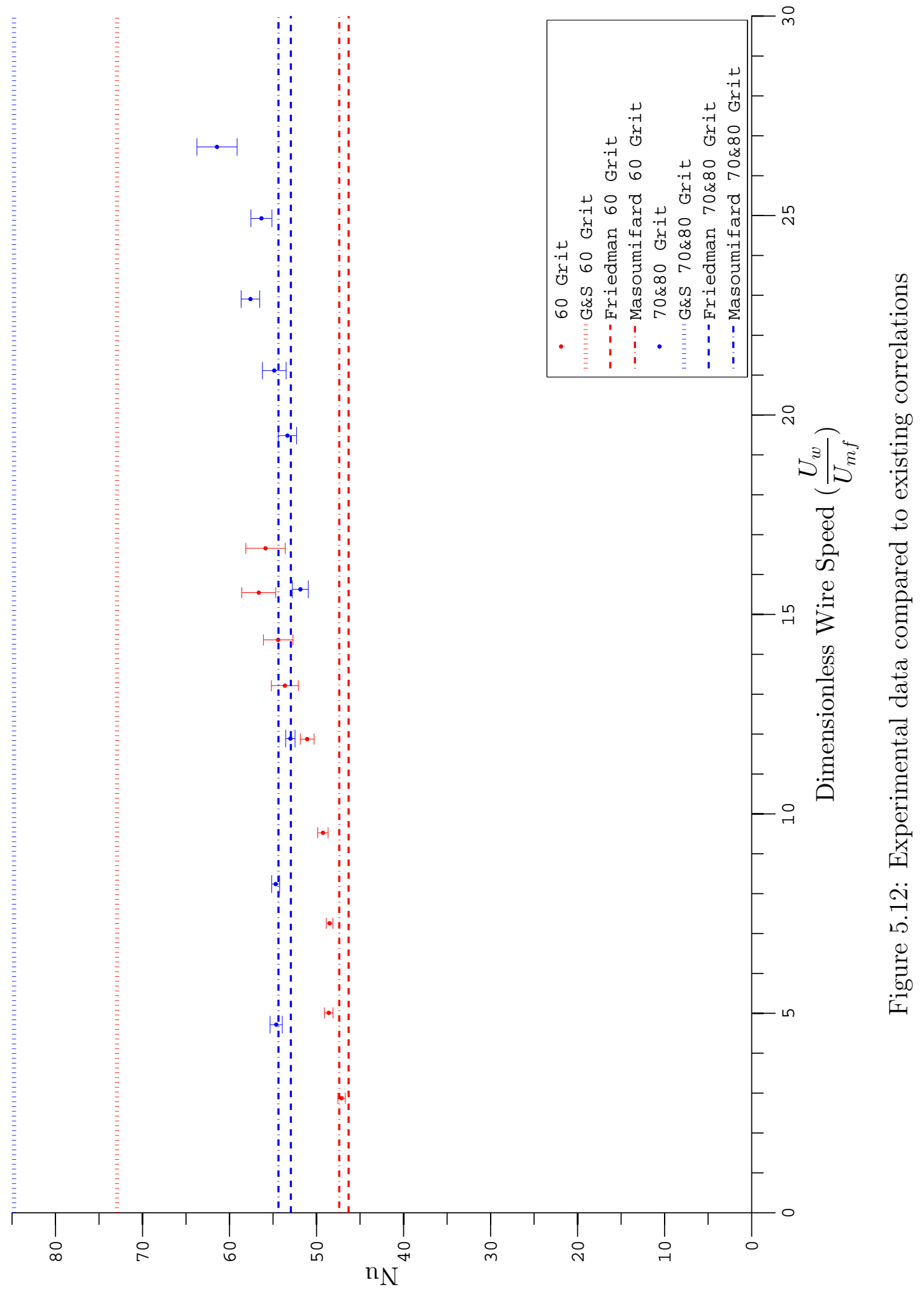




\subsection{Discussion of Results}

\section{New Correlation for Moving Wires}

Since this work is primarily intended for engineering application, and Masoumifard et al.'s correlation requires that all of the properties of the cluster be evaluated, in addition to various other parameters such as bubble fraction, gas gap thickness, and an array of constants, it was deemed too cumbersome to work with. Due to this Friedman et al.'s correlation was taken as a baseline from which to develop the new correlation.

The correlation sought was of the form:

$$
N u_{\text {mean }_{\text {moving }}}=N u_{\text {mean }_{\text {static }}} \times\left[1+\mathrm{C}\left(\frac{u_{w}}{U_{m f}}\right)^{n}\right]
$$

Where $N u_{\text {static }}$ is the Nusselt number found using Friedman et al.'s correlation, Equation 2.12. The two constants were solved for iteratively using Excel's Solver add-on with a goal of minimizing the squared distance from the correlation for both data sets. The values obtained were $\mathrm{C}=0.02$ and $n=0.5$.

The final form of the correlation then became:

$$
N u_{\text {mean }_{\text {moving }}}=\left[1.35 A r^{0.15} \frac{D_{t}}{d_{p}}\right] \times\left[1+0.02\left(\frac{u_{w}}{U_{m f}}\right)^{0.5}\right]
$$

Equation 5.5, also referred to as the Modified Friedman Correlation, was then plotted along with the experimental data and previous correlations for comparison, in Figure 5.13.

Equation 5.5 manages to predict all of the experimental data within a $\pm 15 \%$ range as can be seen from Figure 5.14. While Equation 5.5 is certainly an effective correlation for engineering application, and can approximate the data quite well, it does not reflect the shape of the data very well. The experimental data has an ' $\mathrm{S}$ ' shape, gradually increasing initially, followed by a flat middle portion, with another increase near the end and potentially a plateau after that. The steadily decreasing slope of Equation 5.5 does not properly capture the flat middle portion, and completely misses the increase near the end. Multiple terms would be needed to effectively describe the data, meaning a more complex correlation, and likely more experimental data would be needed to formulate it. 


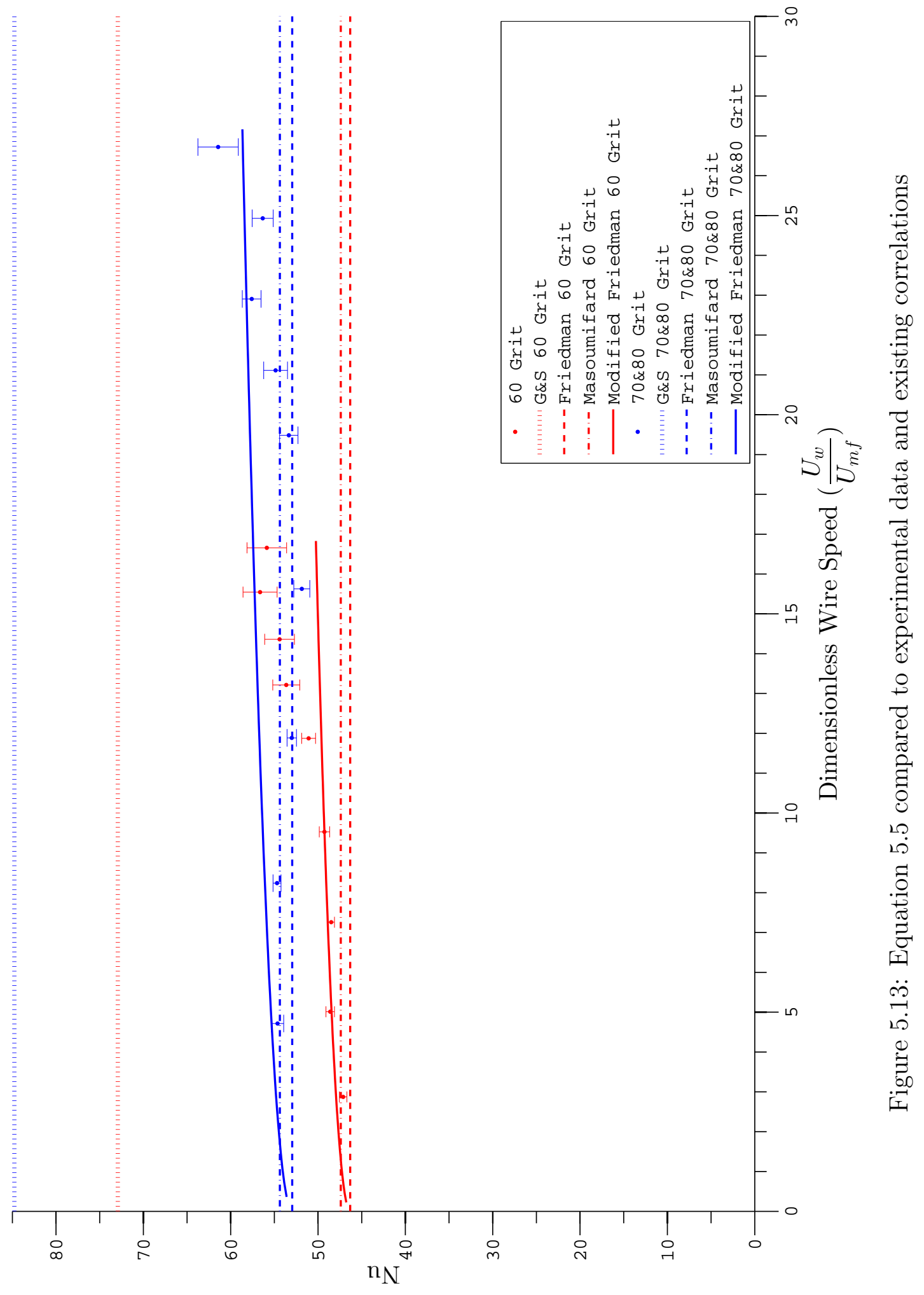




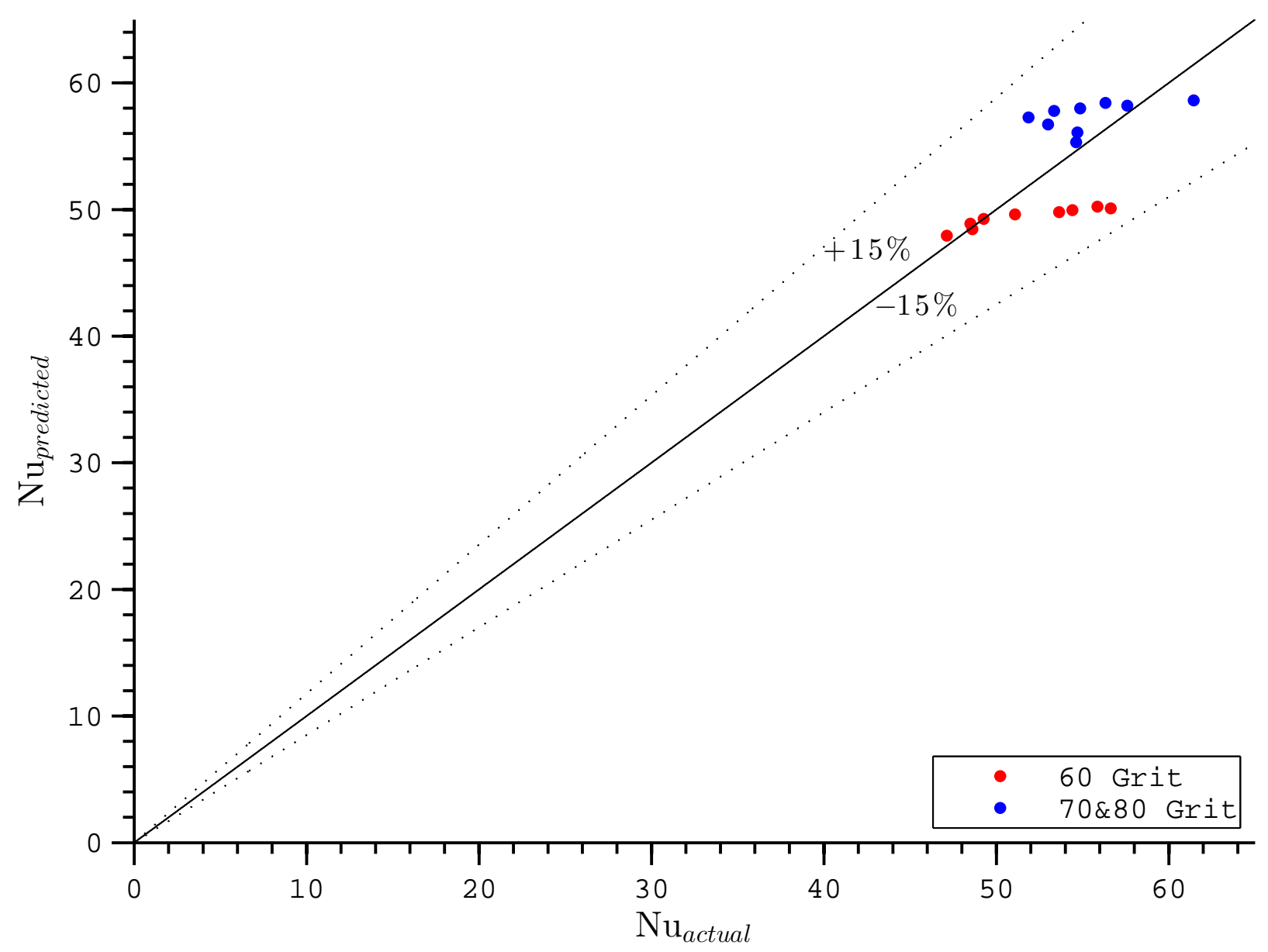

Figure 5.14: Equation 5.5 vs experimental data

\section{Modifying $\tau$ in the Masoumifard Correlation}

The Masoumifard et al. correlation previously presented in the Theoretical Considerations (Equation 2.13) contains a term, $\tau$, that represents the duration of the contact between a cluster of particles and the immersed surface. As can be seen in Equation 5.6, this duration is calculated based on particle diameter, surface diameter, $U_{m f}$ and the ratio of current fluidizing velocity to $U_{m f}$. 


$$
\tau=1.2\left[\frac{d_{p} g}{U_{m f}^{2}\left(\frac{U_{0}}{U_{m f}}\right)^{2}}\right]^{0.3}\left(\frac{d_{p}}{D_{t}}\right)^{0.225}
$$

The introduction of wire movement would play no role on the particle diameter or $U_{m f}$, as these are purely related to particle and gas properties and the wire cannot influence them or cause them to be perceived differently in any way. The wire diameter is perpendicular to the wire motion, so it would not appear any differently regardless of whether the wire was moving or static. The only remaining factor is fluidizing velocity, which also represents the velocity of the cluster. Wire movement would not affect the velocity of the cluster, but it would change the relative velocity between the two.

Since the wire and fluidizing velocities are perpendicular, the most appropriate way to determine their relative velocity is using the Pythagorean Theorem. A new velocity, $u_{\text {comb }}$ was defined, as the vector sum of the wire and fluidizing velocities.

$$
u_{\text {comb }}=\sqrt{U_{0}^{2}+u_{w}^{2}}
$$

This was tested and found to greatly over-predict the Nusselt number, so it was concluded that the wire velocity does not play as large a role as the fluidizing velocity in this correlation. To compensate for this, a constant was added in front of the $u_{w}$ term in Equation 5.7:

$$
u_{c o m b}=\sqrt{U_{0}^{2}+C u_{w}^{2}}
$$

Through the use of Excel's Solver add-on, the constant was found to be 0.275, resulting in:

$$
u_{\text {comb }}=\sqrt{U_{0}^{2}+0.275 u_{w}^{2}}
$$

A plot of Equation 5.9, also referred to as the Modified Masoumifard Correlation, together with Equation 5.5 and the experimental data can be seen in Figure 5.16.

The Modified Masoumifard Correlation (MMC), Equation 5.9 also manages to predict all of the experimental data within a $\pm 15 \%$ range as can be seen from Figure 5.15 , but errs in a 


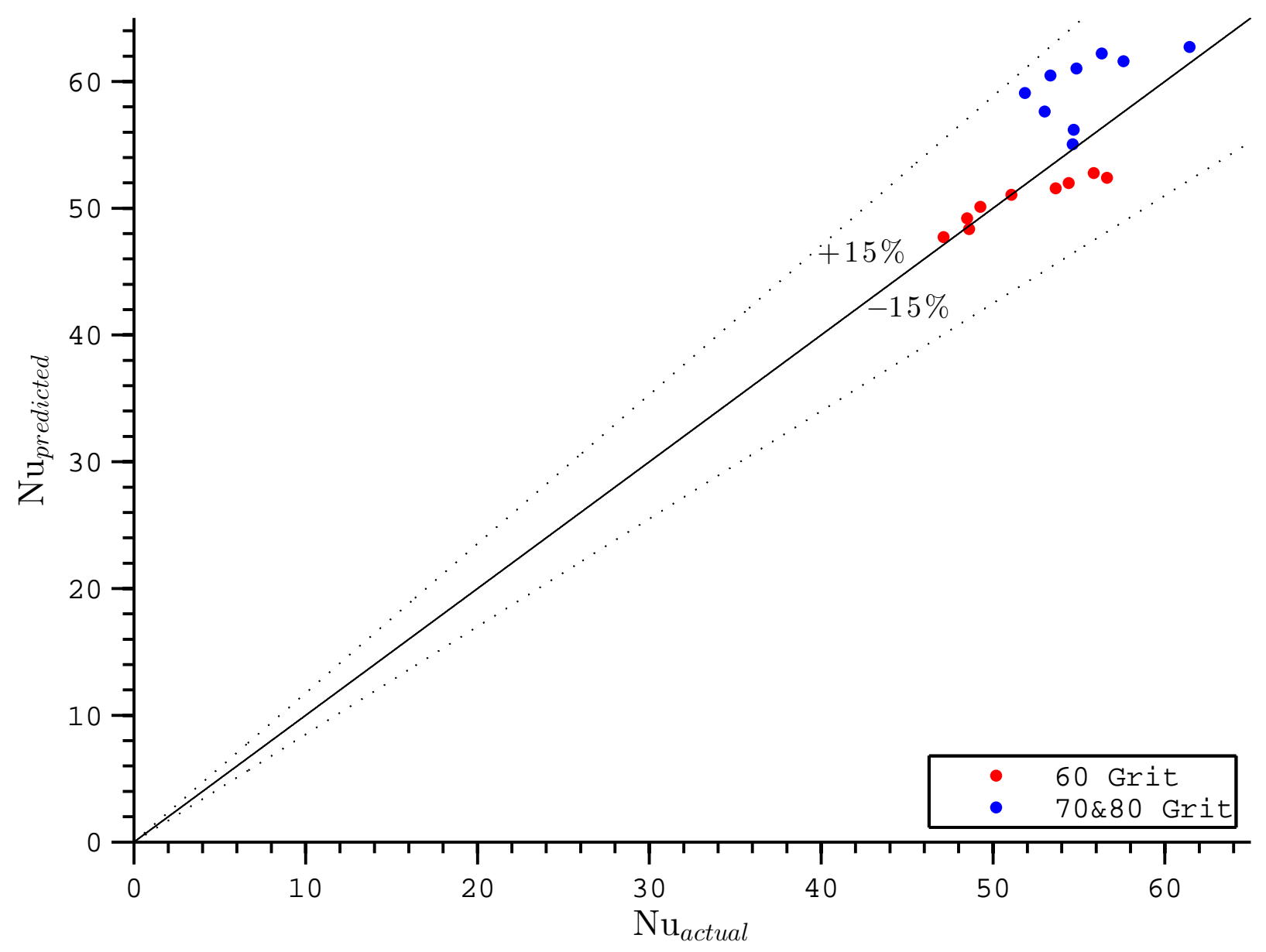

Figure 5.15: Equation 5.9 vs experimental data

different manner than the Modified Friedman Correlation(MFC), Equation 5.5. As can be seen from Figures 5.15 and 5.16, the MMC manages to predict the shape of the data better than the MFC, especially in the case of the 60 Grit data set. It suffers on the $70 \& 80$ Grit mix data set due to the dip in the middle of the data, between a dimensionless wire speed of 8 and 22, the MMC predicts a steady increase at this point which results in the largest discrepancy and harms its overall fit.

Both correlations are useful for different applications; the MFC is well suited to quick calculations and manages to predict the data well, even if it cannot capture its shape perfectly. The MMC has a shape that fits the data much better, but is much more cumbersome to 


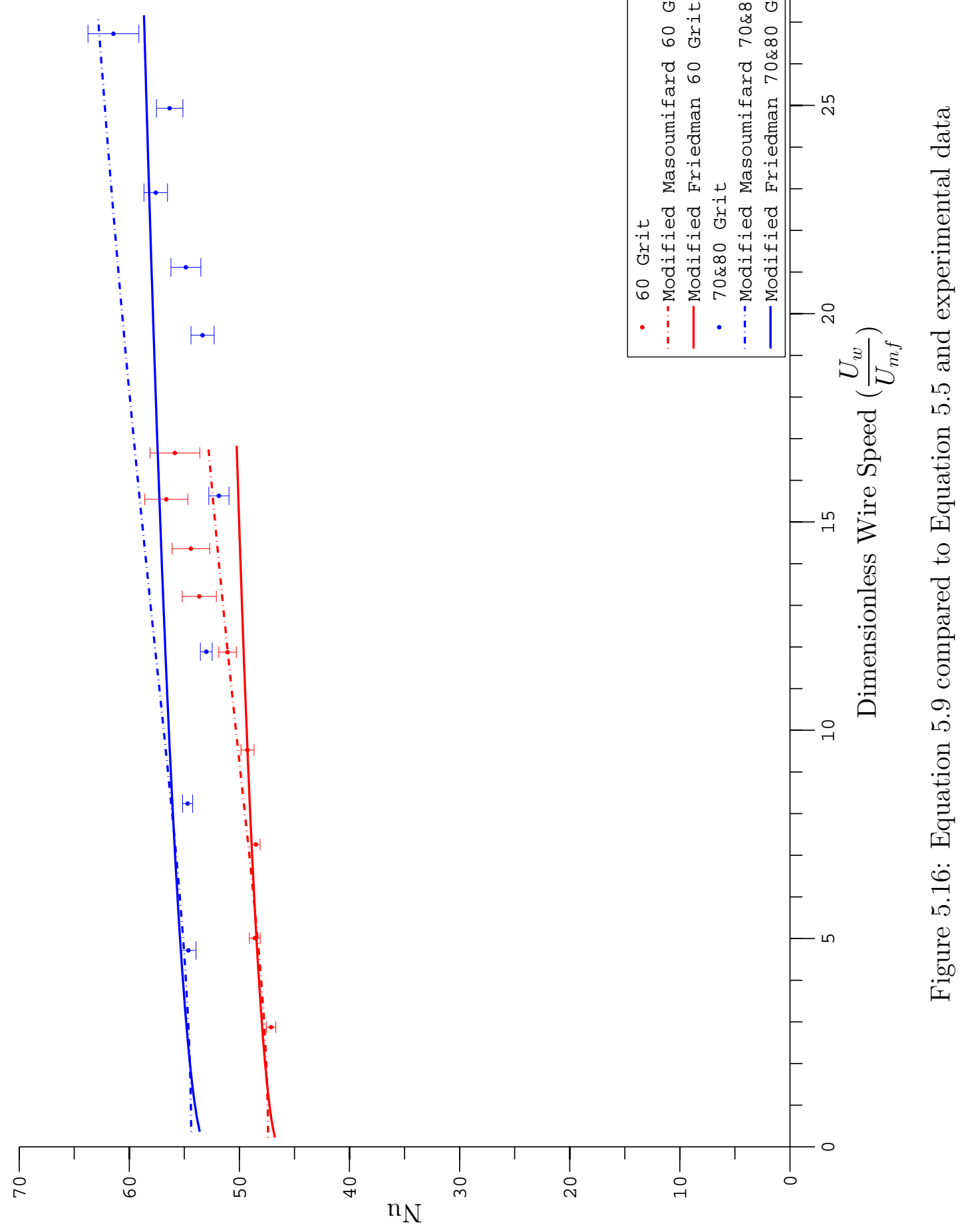


work with and as such is more suited to scientific applications. 


\section{CHAPTER 6}

\section{Error Assessment}

\subsection{Introduction}

This study is primarily focused on heat transfer rates and their relation to wire speeds, but fluidization velocity is also a parameter of interest, albeit to a lesser extent. Previous work by Friedman et al. (2006) and Masoumifard et al. (2008) has shown that for small immersed cylinders, such as the ones in this study, fluidizing velocity does not influence Nusselt number past $2.5 \times U_{m f}$. This was confirmed with a set of tests in the present work. However, to ensure the validity of the tests, it is important to know the fluidizing velocity within a certain threshold.

Heat transfer and wire speed uncertainties are the key focus of this chapter, and will be discussed together, as the wire speed uncertainty affects the heat transfer uncertainty as well.

\subsection{Air Flow Uncertainties}

The Flow Products Venturi utilized for measuring the flow rate has a design pressure drop of $378.46 \mathrm{~mm}$ of water at a flow rate of $254 \mathrm{~m}^{3} / \mathrm{hr}$. The current flow rate can be calculated using the following:

$$
\frac{G}{G_{r e f}}=\sqrt{\frac{\Delta p}{\Delta p_{r e f}}}
$$




\subsection{Air Flow Uncertainties}

$$
\begin{aligned}
\text { where } G & =\text { Measured air flow rate }\left(\frac{m^{3}}{h r}\right) \\
G_{r e f} & =\text { Reference flow rate }\left(254 \frac{m^{3}}{h r}\right) \\
\Delta p & =\text { Measured presure drop (mm of water }) \\
\Delta p_{r e f} & =\text { Reference presure drop }(378.46 \mathrm{~mm} \text { of water })
\end{aligned}
$$

The pressure drop readings are made on a Dwyer Instruments manometer, which reads in inches of water as opposed to $\mathrm{mm}$, and has a precision of $\pm 0.1^{\prime \prime}$ of water, which translates to $\pm 2.54 \mathrm{~mm}$ of water. The formula for calculating error when a value is raised to a power, assuming a small $\Delta x$ is:

$$
\frac{\Delta y}{y} \approx n \frac{\Delta x}{x}
$$

in this formula $y=G$, measured air flow rate

$$
\begin{aligned}
\Delta y & =\text { Error in air flow rate } \\
x & =\frac{\text { Measured pressure drop }}{\text { Reference pressure drop }} \\
\Delta x & =\text { Error in pressure } \operatorname{drop}( \pm 2.54 \mathrm{~mm})
\end{aligned}
$$

The range of measurements for pressure drop in this experiment varied from $30.48 \mathrm{~mm}\left(1.2^{\prime \prime}\right)$ to $101.6 \mathrm{~mm}$ (4"). This gives a range of flow rates from $72.2 \mathrm{~m}^{3} / \mathrm{hr}$ to $132 \mathrm{~m}^{3} / \mathrm{hr}$.

Using these values in Equation 6.2 produces a range of error $\pm 1.95 \mathrm{~m}^{3} / \mathrm{hr}$ for the lower range of flow rates and $\pm 1.65 \mathrm{~m}^{3} / \mathrm{hr}$ for the high. This corresponds to a maximum uncertainty of $2.7 \%$ at lower flow rates and $1.25 \%$ at higher ones.

Since the fluidizing velocity is not used as a parameter in this study, but merely to ensure it is in a desired range, the error ranges in this case are certainly acceptable. 


\subsection{Heat Transfer Uncertainties}

Since the heat transfer is calculated using an iterative numerical scheme, an alternate method for determining error in heat transfer was necessary. Due to the small diameter of the wire, it was theorized that conduction would play a very small role. To confirm this, the thermal conductivity of the wire was varied over a large range in the numerical model, to see what effect it would have on the outlet temperature. As can be seen from Figure 6.1, the minimum outlet temperature predicted was $32.1421^{\circ} \mathrm{C}$ and the maximum was $32.1424^{\circ} \mathrm{C}$, as such the change in outlet temperature is far too small to be detected with the available instruments, and conduction along the wire is essentially negligible in comparison to convection and advection. Due to this, a new, simplified differential equation was found for the purpose of error estimation, by removing the conduction term from the original one. Starting with Equation 4.8:

$$
\frac{d}{d x}\left(\dot{Q}_{x}\right)+\rho_{w} u_{w} C_{p, w} A_{c} \frac{d T}{d x}+h P\left(T(x)-T_{\infty}\right)=0
$$

and eliminating the $\frac{d}{d x}\left(\dot{Q}_{x}\right)$ term results in:

$$
\rho_{w} u_{w} C_{p, w} A_{c} \frac{d T}{d x}+h P\left(T(x)-T_{\infty}\right)=0
$$

Once again defining $\Theta(x)$ as the temperature difference between the bed and wire temperatures, $T(x)-T_{\infty}$ results in:

$$
\rho_{w} u_{w} C_{p, w} A_{c} \frac{d \Theta}{d x}+h P \Theta(x)=0
$$

Substituting $K=\rho_{w} u_{w} C_{p, w} A_{c}$ and $m=h P$ for easier solution then gives:

$$
K \frac{d \Theta}{d x}+m \Theta(x)=0
$$

which was then solved in Maple to give:

$$
\Theta(x)=C_{1} e^{-\frac{m x}{K}}
$$




\subsection{Heat Transfer Uncertainties}

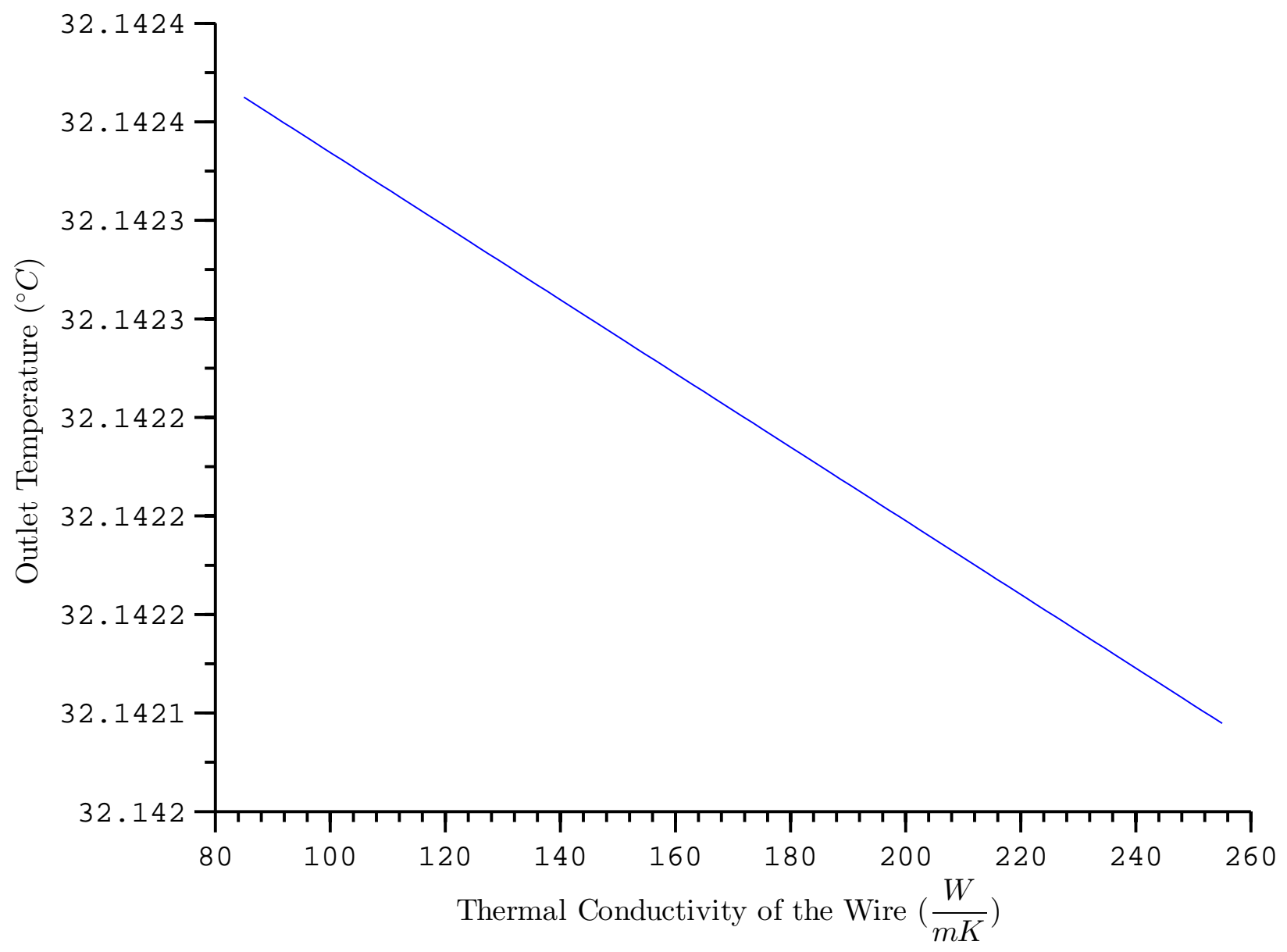

Figure 6.1: Outlet temperature vs wire thermal conductivity

As there is only one constant, a single boundary condition is sufficient to solve for the particular solution, so $\Theta(0)=\Theta_{\text {in }}$ was chosen. The particular solution then becomes:

$$
\Theta(x)=\Theta_{i n} e^{-\frac{m x}{K}}
$$

At the outlet, which is the location of interest, it would become:

$$
\Theta(L)=\Theta_{i n} e^{-\frac{m L}{K}}
$$




\subsection{Heat Transfer Uncertainties}

Restoring the expanded values for $m, K$ and $\Theta(x)$, and noting that $T(L)$ is just $T_{\text {out }}$ gives:

$$
T_{\text {out }}-T_{\infty}=\left(T_{\text {in }}-T_{\infty}\right) e^{-\frac{h P L}{\rho_{w} w_{w} C_{p}, w A_{c}}}
$$

This can then be re-arranged into:

$$
\frac{T_{\text {out }}-T_{\infty}}{T_{\text {in }}-T_{\infty}}=e^{-\frac{h P L}{\rho_{w} u_{w} C_{p, w} A_{c}}}
$$

Taking the natural logarithm of both sides would then result in:

$$
\ln \left(\frac{T_{\text {out }}-T_{\infty}}{T_{\text {in }}-T_{\infty}}\right)=-\frac{h P L}{\rho_{w} u_{w} C_{p, w} A_{c}}
$$

At which point $\mathrm{h}$ can be isolated to give:

$$
h=-\frac{\rho_{w} u_{w} C_{p, w} A_{c}}{P L} \times \ln \left(\frac{T_{o u t}-T_{\infty}}{T_{\text {in }}-T_{\infty}}\right)
$$

It is important to note that both the perimeter, $P$, and the cross sectional area, $A_{c}$ are a function of diameter:

$$
\begin{aligned}
P & =\pi D_{t} \\
A_{c} & =\pi\left(\frac{D_{t}}{2}\right)^{2}
\end{aligned}
$$

Substituting those into Equation 6.12 results in:

$$
h=-\frac{\rho_{w} u_{w} C_{p, w} D_{t}}{4 L} \times \ln \left(\frac{T_{\text {out }}-T_{\infty}}{T_{\text {in }}-T_{\infty}}\right)
$$




\subsection{Heat Transfer Uncertainties}

Which when modified for calculating propagated error becomes the following:

$$
\begin{aligned}
\Delta h=h \times\left[\frac{\left\{\left(\frac{\Delta \rho_{w}}{\rho_{w}}\right)^{2}+\left(\frac{\Delta u_{w}}{u_{w}}\right)^{2}+\left(\frac{\Delta C_{p, w}}{C_{p, w}}\right)^{2}+\left(\frac{\Delta D_{t}}{D_{t}}\right)^{2}+\left(\frac{\Delta L}{L}\right)^{2}\right\}}{\left(-\frac{\rho_{w} u_{w} C_{p, w} D_{t}}{4 L}\right)^{2}}\right. \\
+ \\
\left.+\frac{\left\{\frac{\Delta T_{\text {out }}^{2}+\Delta T_{\infty}^{2}}{\left(T_{\text {out }}-T_{\infty}\right)^{2}}+\frac{\Delta T_{\text {in }}^{2}+\Delta T_{\infty}^{2}}{\left(T_{\text {in }}-T_{\infty}\right)^{2}}\right\}}{\left(\ln \left(\frac{T_{\text {out }}-T_{\infty}}{T_{\text {in }}-T_{\infty}}\right)\right)^{2}}\right]^{1 / 2}
\end{aligned}
$$

The terms $\left(\frac{\Delta \rho_{w}}{\rho_{w}}\right)^{2}$ and $\left(\frac{\Delta C_{p, w}}{C_{p, w}}\right)^{2}$ were dropped because reference values for Aluminum were used, which are given in literature without any associated error, thus $\Delta \rho_{w}$ and $\Delta C_{p, w}$ are zero.

Equation 6.16 was programmed into the Matlab script and used to calculate the error in $\mathrm{h}$. Typical values ranged between a maximum of $\pm 25 \frac{W}{m^{2} K}$ at $475 \frac{W}{m^{2} K}$ which is approximately $5.3 \%$ and $\pm 17 \frac{W}{m^{2} K}$ at $630 \frac{W}{m^{2} K}$ which is approximately $2.7 \%$.

Nusselt number is calculated from h, using Equation 5.2:

$$
N u=\frac{h D_{t}}{k_{g}}
$$

Obtaining the error for Nusselt number then, is as follows:

$$
\Delta N u=N u \times\left\{\left(\frac{\Delta h}{h}\right)^{2}+\left(\frac{\Delta D_{t}}{D_{t}}\right)^{2}+\left(\frac{\Delta k_{g}}{k_{g}}\right)^{2}\right\}^{1 / 2}
$$

The $\left(\frac{\Delta k_{g}}{k_{g}}\right)^{2}$ term was omitted, as the correlation for thermal conductivity of air used has nearly no deviation from experimental values at these ranges of temperature(Stephan and Laesecke, 1985). The values for error in Nusselt number ranged between a maximum of \pm 2.6 at 47 and \pm 2.1 at 61.5 , which is $5.53 \%$ and $3.41 \%$ respectively. 


\subsubsection{Wire Speed Uncertainties}

The Shimpo DT-105A tachometer used in these experiments is incapable of any sort of data logging. It is however capable of displaying the maximum and minimum value for each run, so for determining the uncertainty in the wire speed, the mean value of the two was taken to be the wire speed, and the error was taken as the difference between the mean and the extreme values. While this method does not guarantee that the mean value is the true value, it does guarantee that all values lie inside the range described by the extreme values. This means that the error in wire speed might be overstated, but it cannot be understated.

There is also additional error in the wire speed measurement, from the accuracy of the DT-105A itself, which has been determined to be $\pm 0.39 \mathrm{~m} / \mathrm{min}$. The typical maximum to minimum spread in most scenarios is around $\pm 1.25 \mathrm{~m} / \mathrm{min}$. Due to the size difference in the two forms of error, the statistical spread completely dominates and the final value is very close to it.

\subsection{Non-Quantifiable Uncertainties}

In addition to the uncertainties discussed in the previous sections, there are a few more factors that affect fluidized beds whose impacts are difficult to measure. The most important ones are the chaotic nature of the bubbles and the potential influence of relative humidity. As was mentioned in the Results and Discussion, the higher speed runs, where an element of wire would have a very short residence time inside the bed had a very wide spread, or scatter of heat transfer coefficients. This can be clearly seen in Figures 5.7 and 5.8. The reason for this is the bubbling nature of a fluidized bed. Bubbles form at any point on the distributor and coalesce as they rise. The wire passes through the middle of the bed, and is very small in diameter, so it only takes up $1.84 \%$ of the bed cross sectional area. Since the location of the bubbles keeps changing, and the wire is such a small portion of the cross section, it is exposed to different amounts of bubbling at all times. Combined with the very short 


\subsection{Non-Quantifiable Uncertainties}

residence times and low conduction along the wire, this can mean that each section of wire can experience very high or very low heat transfer rates. Steps were taken to minimize the effect of this, by repeating tests multiple times, however there is no way to guarantee that this was sufficient as it is not known how long a time a wire element would need to be inside the fluidized bed to ensure it has experienced the true average amount of heat transfer.

Another concern is the effect that humidity would have on the results, as the addition of moisture to sand increases the surface tension, causing the sand to stick together. The humidity of the laboratory was not monitored and there is no information in literature on what effect if any humidity has on fluidization, so this uncertainty also cannot be quantified. 


\section{CHAPTER 7}

\section{Conclusions and Recommendations}

\subsection{Conclusions}

The goals set out in the scope of the thesis have been met:

- An experimental apparatus to feed a wire at a constant, controlled speed through a fluidized bed furnace was developed and tested.

- An analytical and a numerical model capable of determining the average heat transfer coefficient and Nusselt number based on available parameters such as inlet, outlet, and bed temperatures, as well as wire speed was developed.

- The effect of wire speed on the average heat transfer coefficient and Nusselt number was measured.

- A simple engineering correlation that can be used to predict the Nusselt number for a moving wire immersed in a fluidized bed furnace was developed and compared with other correlations from the literature.

Two different particle configurations were used in the tests conducted. The first set was analyzed by Koundakjian (2002) in a previous work, the details of which were reported in Section 5.1. The new $70 \& 80$ grit particle mix which was introduced in this work was also analyzed and reported in the same section. They were found to belong to Geldart's Group B, which fluidize easily and exhibit bubbling at slightly above $U_{m f}$.

To effectively determine the average heat transfer coefficient and Nusselt number, the temperature needed to be accurately known. For this purpose the thermometers and DAQ were 


\subsection{Conclusions}

calibrated in a constant temperature bath to a tolerance of $\pm 0.1{ }^{\circ} \mathrm{C}$, the details of which can be seen in Section 5.2. The effect of frictional heating due to wire movement on the thermometers was investigated as well, and found to be negligible.

The effect of fluidization velocity on Nusselt number was also investigated. Previous findings by Friedman et al. (2006) and Masoumifard et al. (2008) indicated that fluidization velocity no longer has a significant impact on Nusselt number at velocities above $2.5 \times U_{m f}$ for a static wire and it was important to determine if this still holds true for a moving wire. Three different fluidization velocities were tested, one below the $2.5 \times U_{m f}$ threshold $\left(2 \times U_{m f}\right)$, and two above $\left(3 \& 4 \times U_{m f}\right)$, at two different wire velocities, $20 \mathrm{~m} / \mathrm{min}$ and $40 \mathrm{~m} / \mathrm{min}$. The details of the tests can be seen in Section 5.3. The findings were confirmed, as a large difference in $\mathrm{Nu}$ between 2 and $3 \times U_{m f}$ was found (13\% on average), while between 3 and $4 \times U_{m f}$ it was only around $5 \%$ and there was noticeable overlap in the error bars from the data sets for $3 \times U_{m f}$ and $4 \times U_{m f}$.

Various wire speeds, ranging from 10-75 m/min were tested in beds of two different particle configurations. The first configuration had a lower, static layer of 8 grit particles and a fludized upper layer of 60 grit particles. The second used the same 8 grit particles at the bottom, but had a mix of 70 and 80 grit particles for the upper fluidized layer. Both sets of data collected exhibited the same general trend of increasing $\mathrm{Nu}$ with increasing wire speed and can be seen in Sections 5.4 and 5.5 respectively. The 60 grit set had a steadier, more linear shape, while the $70 \& 80$ grit mix set showed a pronounced decrease in the middle and a sharp recovery after.

Due to the fact that the dwell time of an element of wire inside the fluidized bed decreases with increasing wire speed, and the stochastic nature of the bubbling inside a fluidized bed, the statistical scatter of the data increased with wire speed for all data sets. This issue was

compounded by the short length of the bed in the experimental apparatus but would be nearly nonexistent in industry, due to the fact that typical bed length is on the order of 20 $m$ vs $0.965 m$ for the one used in this study.

The experimental data was also compared to existing correlations by Grewal and Saxena, Friedman et al. and Masoumifard et al. in Section 5.6.2. Confirming the findings of Friedman 


\subsection{Conclusions}

et al. (2006), the correlation by Grewal and Saxena greatly over predicted the Nusselt number as it was developed for medium and large sized cylinders. The other two correlations matched the experimental data well at low wire speeds, but as they were developed for static wires, could not predict the increases due to wire speed.

A new correlation was developed, by modifying the one developed by Friedman et al. (2006) to include a term for dimensionless wire speed, resulting in Equation 5.5:

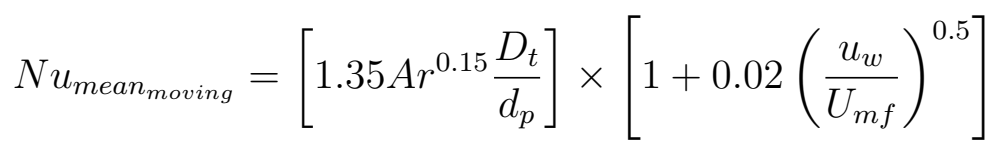

This correlation was able to predict all of the experimental data within a $\pm 15 \%$ margin, however, due to its simple form it was not capable of predicting the shape of the data.

The Masoumifard correlation was also modified by defining a new fluidizing velocity based on the vector sum of the fluidizing velocity and wire speed. It was found that wire speed does not play as large of a role as fluidizing velocity, so a constant was added to the wire speed in order to scale it to match the experimental data. This modification resulted in a shape that matches the experimental data better than the Modified Friedman Correlation presented earlier, however, it was not able to predict the dip in the middle of the $70 \& 80$ grit mix data, which may be an anomaly, and required that many additional parameters and constants be determined prior to use, making it much more difficult to work with than the Modified Friedman Correlation. Both of these correlations can be found in their respective sub-headings in Section 5.6.2.

The knowledge obtained in this study can be of great use in both industry and research. The Modified Friedman Correlation improves upon previous existing correlations, and allows engineers in the field to estimate the heat transfer coefficient to a moving wire in a fluidized bed furnace with greater accuracy than before. Furthermore, the experimental data, as well as the Modified Masoumifard correlation highlights the relationship between wire speed and heat transfer coefficient and can serve as a foundation for future work. 


\subsection{Recommendations}

This work was the first to develop and implement a wire movement system in a fludized bed and the first to characterize a connection between wire movement and heat transfer rates. As in all pioneering efforts, it has a few shortcomings that can be improved upon in future works.

\subsubsection{Apparatus}

Since this apparatus is the first of its kind, there were certain issues and difficulties that could not be predicted in the design process. If corrected the usability and functionality of the apparatus would be greatly improved.

The chief of these issues is the manual rewinding system. Currently, rewinding the apparatus requires two operators to be present, is very physically taxing and results in a poor quality wind compared to what is obtained on the pulling side due to the lack of a wire traverse. Future efforts should take care to include a gear motor, similar to those found on the pulling side, with power in the 0.5 -1hp range and a simpler, more affordable speed controller as the VFDs on the pulling side are excessive for the simple task of rewinding. A wire traverse system is also needed, to ensure the winding quality is high, as any problems due to poor winding on the payoff side appear as spikes in the data and can potentially damage the rest of the apparatus as well. The same linear traverse that is used on the pulling side would be well suited to this purpose. With these additions, a longer wire can be used, which would allow more experiments to be conducted before rewinding, improving the productivity of the apparatus and allowing more tests to be carried out in a single day.

Another important issue is the wire straightener. A single plane unit was purchased and fitted to this system, but after using it for several weeks, it became obvious that a dual plane would be better suited to this purpose. It would ensure a straighter wire, better contact on the thermometers and as such less fluctuations in the results. 


\subsection{Recommendations}

Additionally, a computer interface for the VFDs on the pulling side is needed. Despite the fact that the torque on the collecting spool is set to be lower than the driving spool, it is still capable of overloading the motor on the driving spool if the difference in diameters becomes sufficiently large, and as such requires constant monitoring and occasional adjustment by the operator. With computer based control added, the excess tension could be easily detected, and the speed of the collecting spool could be automatically reduced to relax it, eliminating the need for operator interference.

Finally, the Anritsu contact thermocouples have wearing surfaces that are consumable and need occasional replacement. Unfortunately, Anritsu requires that the thermocouples be sent to their office in Japan for service. For this purpose, two sets of thermocouples should be purchased, as the turn around time for repairing a set is over a month, and no experiments can be conducted if there is no spare.

\subsubsection{Studies}

There is some concern about the validity of the dip in the middle of the $70 \& 80$ grit data. As mentioned in previous chapters, instantaneous heat transfer rates in fludized beds are quite chaotic and extensive repetition or very long fluidized beds are needed to ensure the average determined is the correct one. It is a virtual impossibility to find the kind of floor space in an academic environment for the installation of an industrial scale fluidized bed furnace, but the same effect can be replicated by conducting a very large number of tests. The suggestions in the previous section on modifications to the apparatus would enable this by allowing greater lengths of wire to be used and longer duration tests to be carried out. With the current system, it is far too difficult to maintain a stable and constant wire speed for a long duration, and multiple manual rewinds are far too physically taxing to allow the desirable number of tests to be conducted. It is possible to carry out fewer tests in a single day and span the experiments over many days, but then there are concerns with ambient humidity and temperature which changes on a day to day basis. 


\subsection{Recommendations}

Additionally, it is desirable to study more parameters to gain additional insight into the inter-dependence of various factors. A more extensive study of fluidization velocity in beds of different particles should be conducted to investigate if the $2.5 \times U_{m f}$ holds for every wire speed and particle size, or if it varies with those parameters. Multiple particle sizes and wire diameters should be studied at different wire speeds as well, to see if these parameters have any inter-dependence with wire speed.

These additional studies would allow parameters that have no influence to be eliminated with greater certainty and the rest to be studied in more depth. This would also allow a correlation to be developed that better captures the shape of the data. 


\section{References}

Andeen, B. and Glicksman, L. Heat transfer to horizontal tubes in shallow fluidized beds. In ASME-AIChE Heat Transfer Conference, St Louis, Missouri, pages 9-11. 1976.

Baskakov, A.u., Berg, B., Vitt, O., Filippovsky, N., Kirakosyan, V., Goldobin, J., and Maskaev, V. Heat transfer to objects immersed in fluidized beds. Powder Technology, 8(5):273-282, 1973.

Berg, B. and Baskakov, A. Experimental investigation of heat transfer between a fluidized bed and a cylindrical surface. Journal of engineering physics, 11(1):27-30, 1966.

Boerefijn, R., Poletto, M., and Salatino, P. Analysis of the dynamics of heat transfer between a hot wire probe and gas fluidized beds. Powder technology, 102(1):53-63, 1999.

Botterill, J.S.M. Fluid-bed heat transfer. gas-fluidized bed behaviour and its influence on bed thermal properties. 1975.

Botterill, J. and Desai, M. Limiting factors in gas-fluidized bed heat transfer. Powder Technology, 6(4):231-238, 1972.

Carman, P. Fluid flow through granular beds. Transactions-Institution of Chemical Engineeres, 15:150-166, 1937.

Company, A.E.C. Evaluation of fluid bed heat exchanger optimization parameters, final technical report submitted to the department of energy under contract no. ac03-78etii343. 1980.

DataTranslation. DT9282 Data Sheet, 2014a.

DataTranslation. DT9282 User's Manual, 2014b.

Ergun, S. Fluid flow through packed columns. Chem. Eng. Prog., 48, 1952. 
Friedman, J., Koundakjian, P., Naylor, D., and Rosero, D. Heat transfer to small horizontal cylinders immersed in a fluidized bed. Journal of heat transfer, 128(10):984-989, 2006.

Geldart, D. Types of gas fluidization. Powder technology, 7(5):285-292, 1973.

Geldart, D. Gas fluidization technology. John Wiley and Sons Inc., New York, NY, 1986.

Grewal, N. A correlation for maximum heat transfer coefficient between a horizontal tube and a gas-solid fluidized bed of small particles. Letters in Heat and Mass Transfer, 9(5):377384, 1982.

Grewal, N. and Saxena, S. Heat transfer between a horizontal tube and a gas-solid fluidized bed. International Journal of Heat and Mass Transfer, 23(11):1505-1519, 1980.

Grewal, N.S. and Saxena, S.C. Maximum heat transfer coefficient between a horizontal tube and a gas-solid fluidized bed. Industrial $\&$ Engineering Chemistry Process Design and Development, 20(1):108-116, 1981.

Gupta, C.K. and Sathiyamoorthy, D. Fluid bed technology in materials processing. CRC Press, 1998.

Holdich, R.G. Fundamentals of particle technology. Midland Information Technology and Publishing, 2002.

Jaluria, Y. and Singh, A. Temperature distribution in a moving material subjected to surface energy transfer. Computer methods in applied mechanics and engineering, 41(2):145-157, 1983.

Karwe, M. and Jaluria, Y. Experimental investigation of thermal transport from a heated moving plate. International journal of heat and mass transfer, 35(2):493-511, 1992.

Koundakjian, P. Heat Transfer to Small Cylinders Immersed in a Fluidized Bed Heat Treating Furnace. Master's thesis, University of Waterloo, 2002. 
Kozeny, J. Ueber kapillare leitung des wassers im boden. Sitzungsber. Akad. Wiss. Wien, 136:271-306, 1927.

Kunii, D. and Levenspiel, O. Fluidization engineering. RE Krieger Pub. Co.(Huntington, NY), 1977.

Martin, H. Heat transfer between gas fluidized beds of solid particles and the surfaces of immersed heat exchanger elements, part ii. Chemical Engineering and Processing: Process Intensification, 18(4):199-223, 1984.

Masoumifard, N., Mostoufi, N., Hamidi, A.A., and Sotudeh-Gharebagh, R. Investigation of heat transfer between a horizontal tube and gas-solid fluidized bed. International Journal of Heat and Fluid Flow, 29(5):1504-1511, 2008.

Masoumifard, N., Mostoufi, N., and Sotudeh-Gharebagh, R. Prediction of the maximum heat transfer coefficient between a horizontal tube and gas-solid fluidized beds. Heat Transfer Engineering, 31(10):870-879, 2010.

Mickley, H. and Fairbanks, D.F. Mechanism of heat transfer to fluidized beds. AIChE Journal, 1(3):374-384, 1955.

Molerus, O. and Wirth, K.E. Heat transfer in fluidized beds, volume 11. Springer, 1997.

Petrie, J., Freeby, W., and Buckham, J. Heat transfer from horizontal tube bundles to air-fluidized beds. In 60th Annual AICHE Meeting, New York, NY. 1967.

Roy Choudhury, S. and Jaluria, Y. Analytical solution for the transient temperature distribution in a moving rod or plate of finite length with surface heat transfer. International journal of heat and mass transfer, 37(8):1193-1205, 1994.

Sathiyamoorthy, D. and Rao, M.R. Prediction of maximum heat transfer coefficient in gas fluidized bed. International journal of heat and mass transfer, 35(5):1027-1034, 1992. 
Saxena, S. Heat transfer from a bank of immersed horizontal smooth tubes in a fluidized bed. Letters in Heat and Mass Transfer, 6(3):225-229, 1979.

Saxena, S. Heat transfer between immersed surfaces and gas-fluidized beds. Advances in heat transfer, 19(2):97-190, 1989.

Simons, R.E. Simplified formula for estimating natural convection heat transfer coefficient on a flat plate. Electronics Cooling, 2001.

Stephan, K. and Laesecke, A. The thermal conductivity of fluid air. Journal of physical and chemical reference data, 14(1):227-234, 1985.

Vreedenberg, H. Heat transfer between a fluidized bed and a horizontal tube. Chemical Engineering Science, 9(1):52-60, 1958.

Zabrodsky, S.S. Hydrodynamics and heat transfer in fluidized beds. The M.I.T. Press, 1966. 
Appendices 
APPENDIX A

\section{List of Publications}

\section{Conference Papers:}

Tannas A., Naylor D., and Friedman J., Heat Transfer to a Moving Wire in a Gas Fluidized Bed. Presented at 7th International Conference on Thermal Engineering: Theory and Applications, May 2014. 


\section{APPENDIX B}

\section{Matlab Scripts and Functions}

Main Script for processing data:

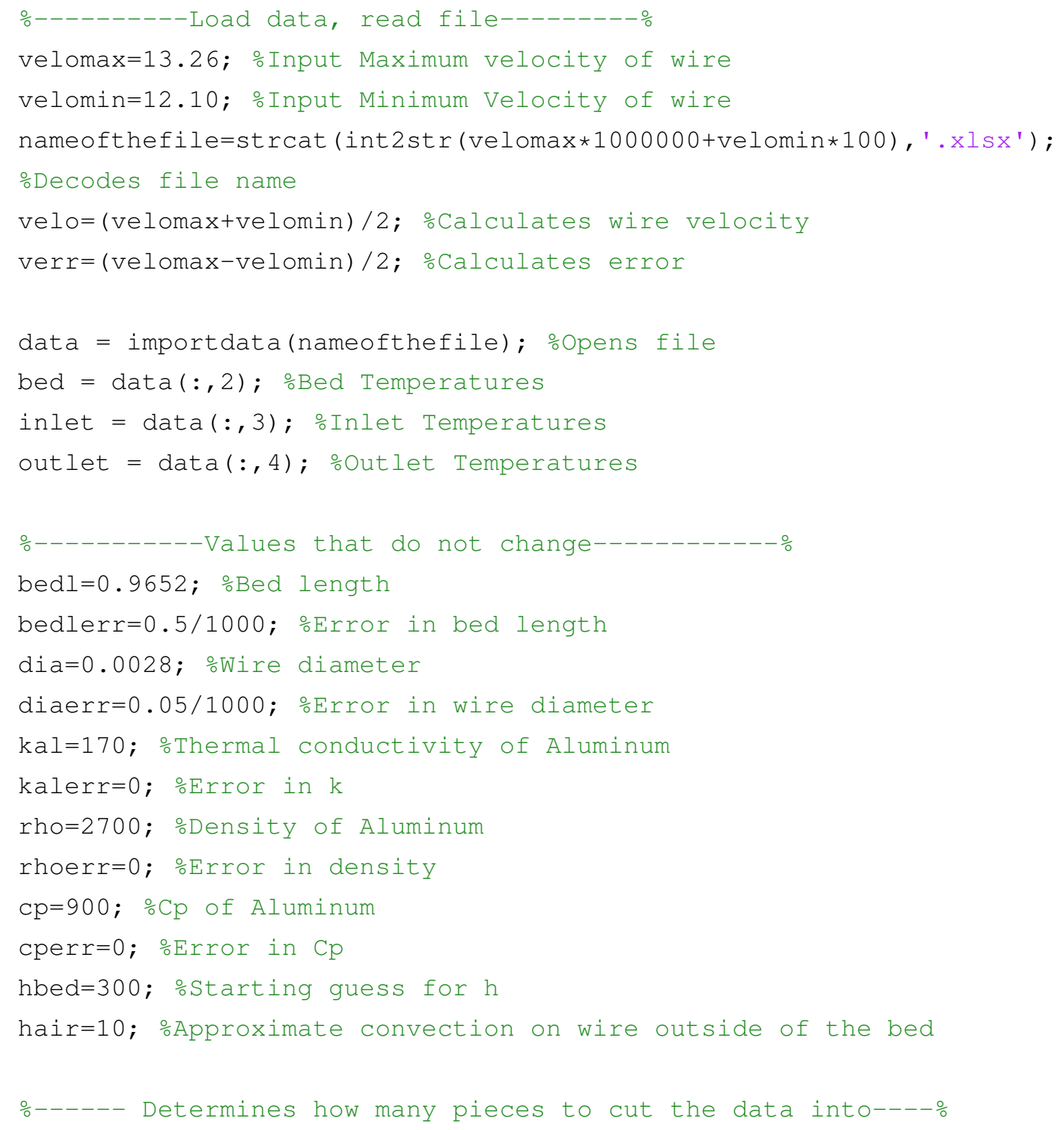




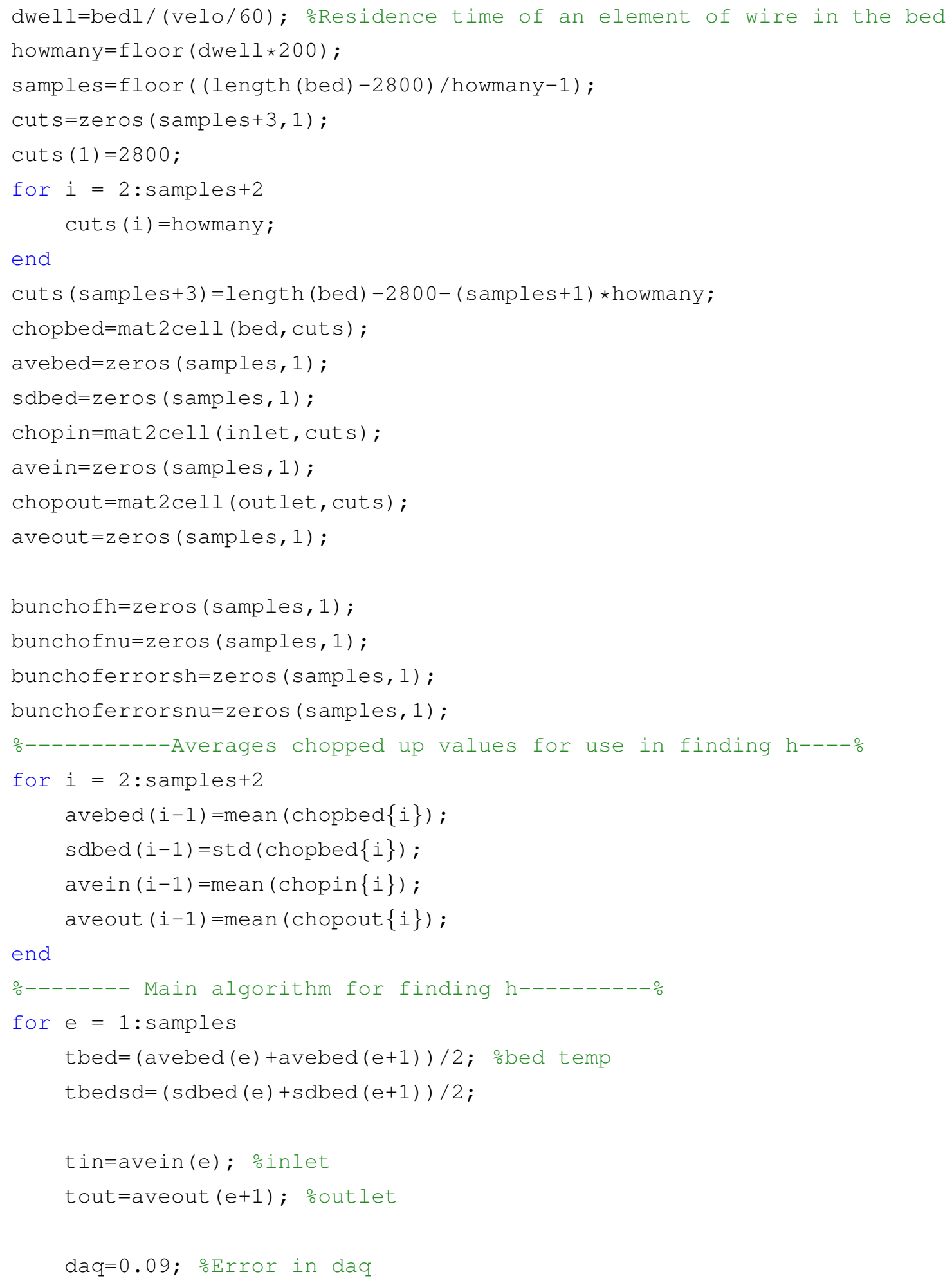




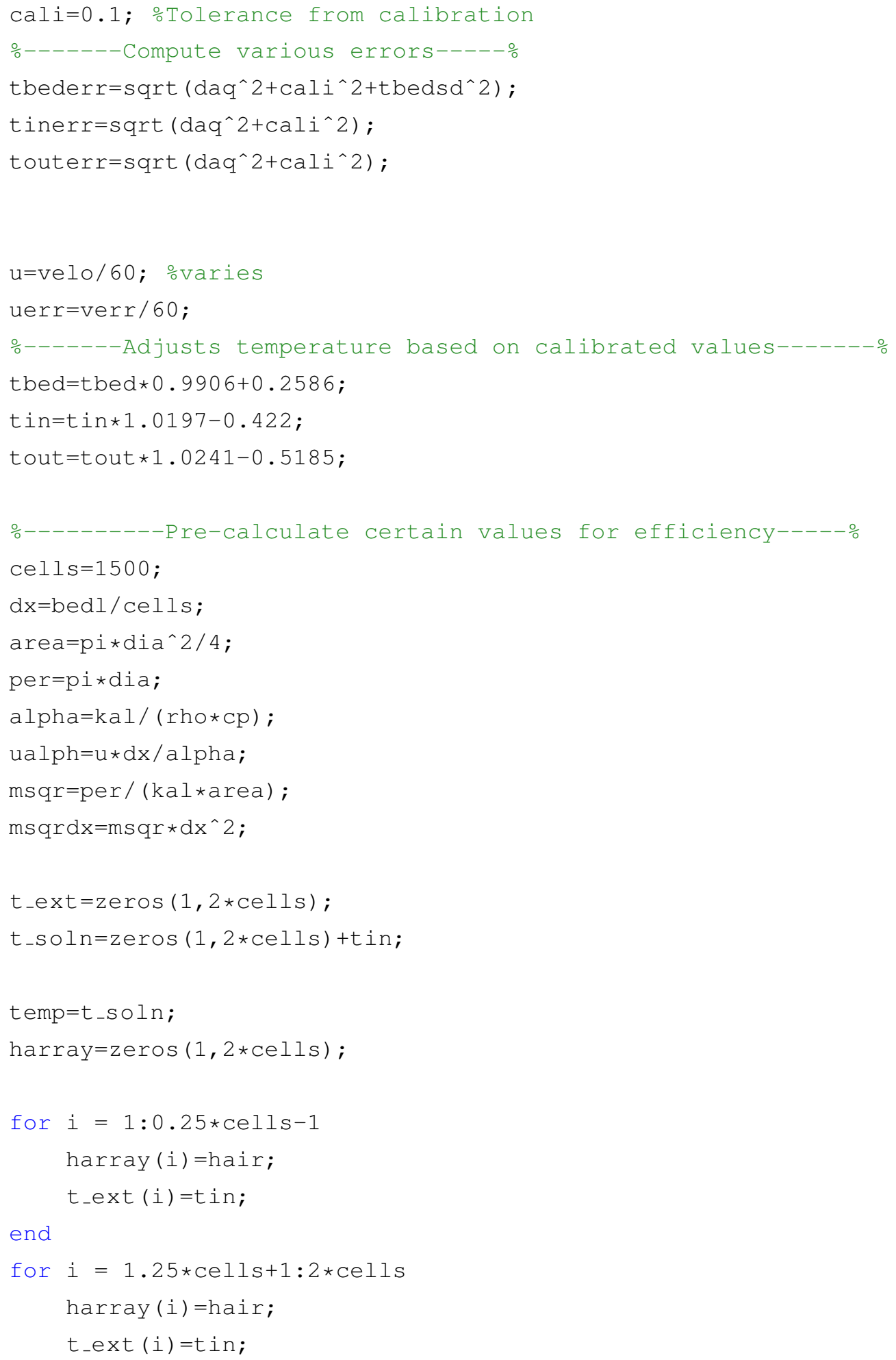


end

stopper $=1$;

while stopper $>0.01$

for $i=0.25 * \operatorname{cells}: 1.25 * \operatorname{cell} \mathrm{s}$

harray $(i)=$ hbed;

t_ext $(i)=$ tbed;

end

stop=1;

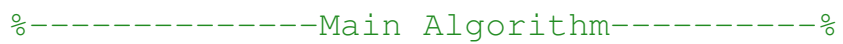

while stop $>0.001$

stop $=0$;

for $i=2: 2 * \operatorname{cells}-1$

temp $(i)=\left(\left(1+u_{a l p h}\right) * t_{-} \operatorname{soln}(i-1)+t_{-} \operatorname{soln}(i+1)+\operatorname{msqr} d x * \ldots\right.$ harray (i)*t_ext (i)) / (2+ualph+msqrdx*harray (i)); stop=stop+abs (t_soln (i) -temp (i));

t_soln (i) =temp (i);

end

$t_{-} \operatorname{soln}(2 * \operatorname{cell} s)=2 * t_{\text {_soln }}(2 * \operatorname{cells}-1)-t_{\text {_soln }}(2 * \operatorname{cells}-2)$; stop;

end

chek=tout-max (t_soln);

hbed=hbed + chek $* 10$;

stopper=abs (chek) Display stopping criteria

end

film $=($ (tin+tout) $/ 2+$ tbed $) / 2$;

thermie=thermcond (film+273.15);

bunchofh $(e)=($ hbed-chek $* 10)$;

bunchofnu $(e)$ =bunchofh $(e) *$ dia/thermie;

응---------Error calculation-------- 응

vall=log (tout/tin);

val2 $=r h o * u * c p * d i a / 4 /$ bedl;

errl=sqrt ( (touterr/tout )^2+(tinerr/tin)^2);

$\operatorname{err} 2=\operatorname{sqrt}\left((\text { rhoerr/rho })^{\wedge} 2+(\text { uerr/u })^{\wedge} 2+(\operatorname{cperr} / \mathrm{cp})^{\wedge} 2+(\text { diaerr/dia })^{\wedge} 2+\ldots\right.$

(bedlerr/bedl)^2)*val2; 
Function for calculating the density of air:

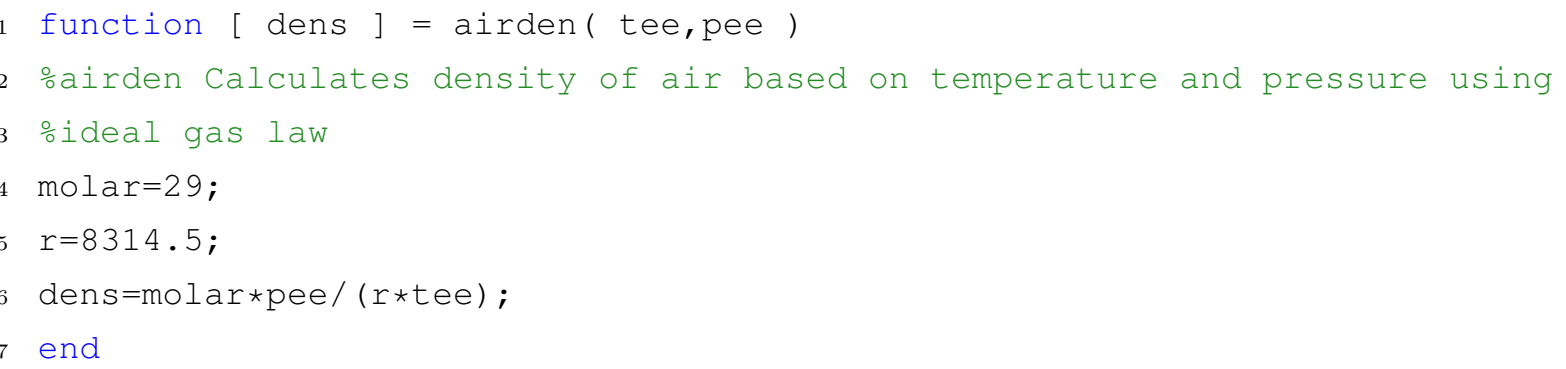

Function for calculating the thermal conductivity of air:

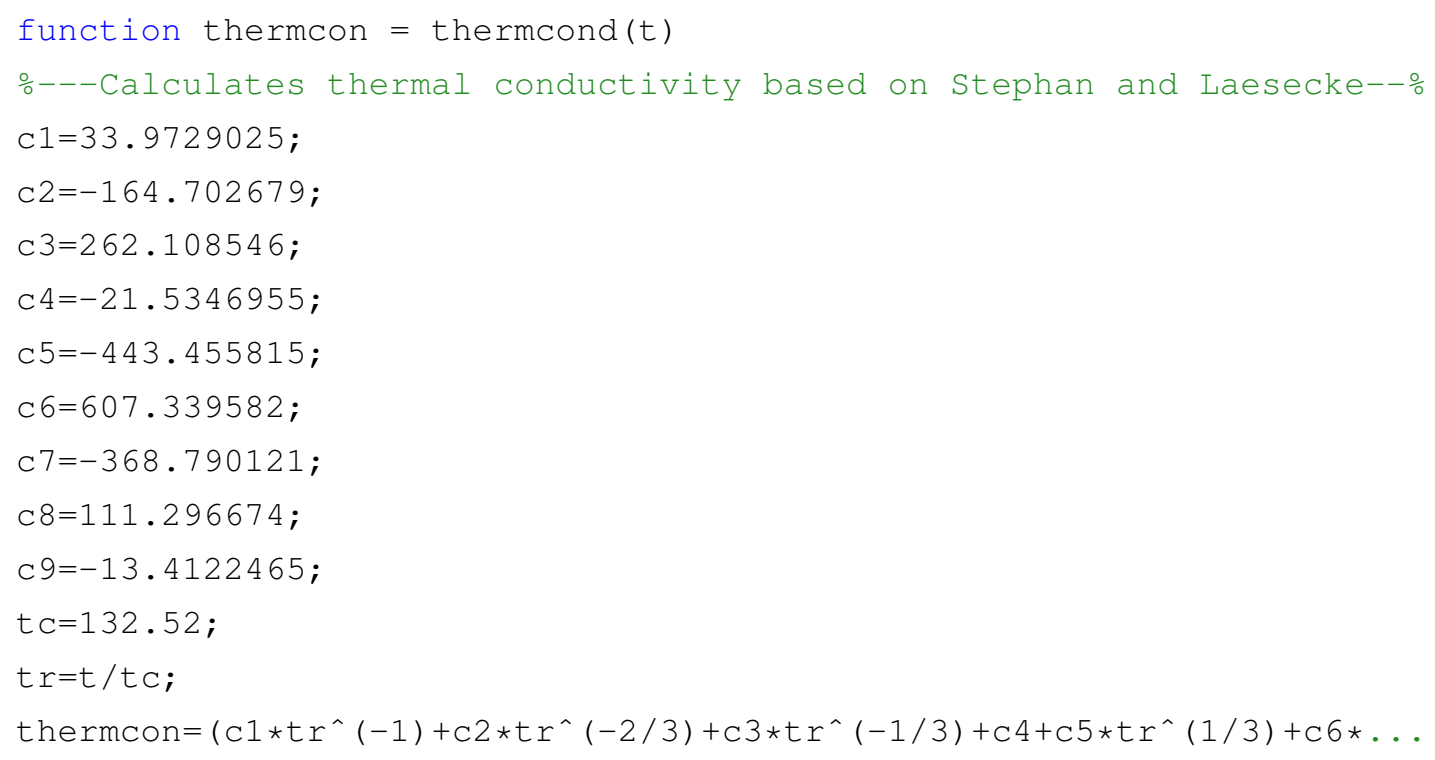


$\left.\operatorname{tr}^{\wedge}(2 / 3)+c 7 * \operatorname{tr}+c 8 * \operatorname{tr}^{\wedge}(4 / 3)+c 9 * \operatorname{tr}^{\wedge}(5 / 3)\right) *\left(4.358 * 10^{\wedge}(-3)\right)$; 